\title{
A stochastic model of hippocampal synaptic plasticity with geometrical readout of enzyme dynamics
}

\author{
Yuri E. Rodrigues ${ }^{1,2,3, *}$, Cezar M. Tigaret ${ }^{4}$, Hélène Marie ${ }^{1,3,6}$, Cian O’Donnell ${ }^{5,6}$, and Romain Veltz ${ }^{2,6, *}$
}

Discovering the rules of synaptic plasticity is an important step for understanding brain learning. Existing plasticity models are either 1) top-down and interpretable, but not flexible enough to account for experimental data, or 2) bottom-up and biologically realistic, but too intricate to interpret and hard to fit to data. We fill the gap between these approaches by uncovering a new plasticity rule based on a geometrical readout mechanism that flexibly maps synaptic enzyme dynamics to plasticity outcomes. We apply this readout to a multi-timescale model of hippocampal synaptic plasticity induction that includes electrical dynamics, calcium, CaMKII and calcineurin, and accurate representation of intrinsic noise sources. Using a single set of model parameters, we demonstrate the robustness of this plasticity rule by reproducing nine published $e x$ vivo experiments covering various spike-timing and frequency-dependent plasticity induction protocols, animal ages, and experimental conditions. The model also predicts that in vivo-like spike timing irregularity strongly shapes plasticity outcome. This geometrical readout modelling approach can be readily applied to other excitatory or inhibitory synapses to discover their synaptic plasticity rules.

To understand how brains learn, we need to identify the rules governing how synapses change their strength in neural circuits. What determines whether each synapse strengthens, weakens, or stays the same? The dominant principle at the basis of current models of synaptic plasticity is the Hebb postulate ${ }^{1}$ which states that neurons with correlated electrical activity strengthen their synaptic connections, while neurons active at different times weaken their connections. In particular, spike-timingdependent plasticity (STDP) models ${ }^{2-4}$ were formulated based on experimental observations that precise timing of pre- and postsynaptic spiking determines whether synapses are strengthened or weakened $^{5-8}$. However, experiments also found that plasticity induction depends on the rate and number of stimuli presented to the synapse $^{9,10}$, and the level of dendritic spine depolarisation ${ }^{11-15}$. The lack of satisfactory plasticity models based solely on neural spiking prompted researchers to consider more elaborate models based on synapse biochemistry ${ }^{16}$. Following a proposed role for postsynaptic calcium $\left(\mathrm{Ca}^{2+}\right)$ signalling in synaptic plasticity ${ }^{17}$, previous models assumed that the amplitude of postsynaptic calcium controls long-term alterations in synaptic strength, with moderate levels of calcium causing long-term depression (LTD) and high calcium causing long-term potentiation (LTP) ${ }^{18,19}$. Recent experimental data suggests that calcium dynamics is also important $^{20-24}$. As a result, subsequent phenomenological models of plasticity incorporated slow variables that integrate the fast synaptic input signals, loosely modelling calcium and its downstream effectors ${ }^{25-32}$.

However, even these models do not account for data showing that plasticity is highly sensitive to physiological conditions such as the developmental age of the animal ${ }^{33-36}$, extracellular calcium and magnesium concentrations ${ }^{37,38}$ and tissue temperature ${ }^{39-41}$. The fundamental issue is that the components of these phenomenological models do not directly map to biological components of synapses, so they cannot automatically model alterations due to physiological and experimental conditions. This absence limits the predictive power of existing plasticity models.

To tackle this problem, we devised a new plasticity rule based on a bottom-up, data-driven approach by building a biologicallygrounded model of plasticity induction at a single rat hippocampal CA3-CA1 synapse. We focus on this synapse type because of the abundant published experimental data that can be used to quantitatively constrain the model parameters. Compared to previous models in the literature, we aimed for an intermediate level of detail: enough biophysical components to capture the key dynamical processes underlying plasticity induction, but not the full molecular cascade underlying plasticity expression; much of which is poorly quantified ${ }^{42}$. Our model centred on dendritic spine electrical dynamics, calcium signalling and immediate downstream molecules, which we then mapped to synaptic strength change via an conceptually new dynamical, geometric readout mechanism. Crucially, the model also captures intrinsic noise based on the stochastic switching of synaptic receptors and ion channels ${ }^{43,44}$. We found that the model can account for published data from spike-timing and frequency-dependent plasticity experiments, and variations in physiological parameters influencing plasticity outcomes. We also tested how the model responded to in vivo-like spike timing jitter and spike failures, and found that the plasticity rules were highly sensitive to these subtle input alterations.

\footnotetext{
${ }^{1}$ Université Côte d'Azur, Nice, Alpes-Maritimes, France. ${ }^{2}$ Institut national de recherche en informatique et en automatique (INRIA), Sophia Antipolis, France. ${ }^{3}$ Institut de Pharmacologie Moléculaire et Cellulaire (IPMC), Valbonne, France. ${ }^{4}$ Neuroscience and Mental Health Research Institute, Division of Psychological Medicine and Clinical Neurosciences, School of Medicine, Cardiff University, Cardiff, UK. ${ }^{5}$ Computational Neuroscience Unit, School of Computer Science, Electrical and Electronic Engineering, and Engineering Mathematics, University of Bristol, Bristol, UK. ${ }^{6}$ Co-last authors. ${ }^{*}$ Corresponding authors: yuri.rodrigues@inria.fr, romain.veltz@inria.fr
} 


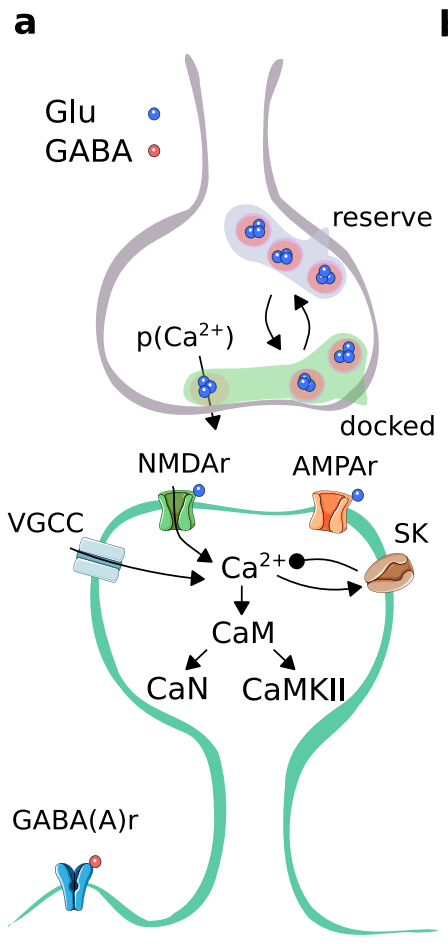

b

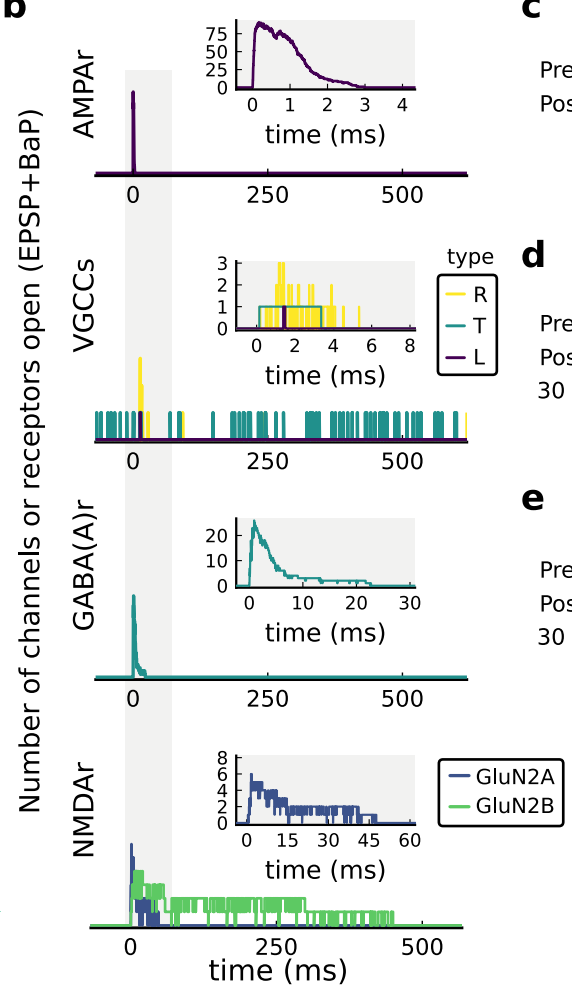

d
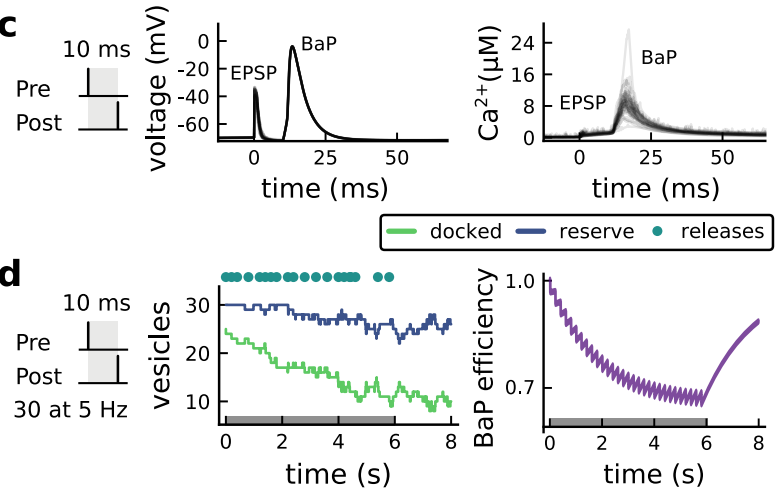

$10 \mathrm{~ms}$

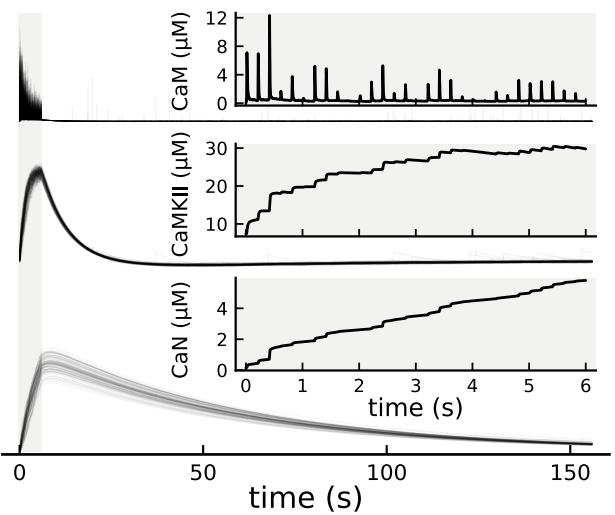

Fig. 1. I The synapse model, its timescales and mechanisms. a, Model diagram with the synaptic components, the pre and postsynaptic compartments. Inhibitory receptor bottom left. b. Stochastic dynamics of the different receptors and channels. Plots show the total number of open channels/receptors as a function of time. AMPArs and NMDArs are activated by glutamate, VGCC are activated by membrane potential, and GABArs are activated by GABA. The timescale of variable response increases from top to bottom panels. c, Dendritic spine membrane potential (left) and calcium concentration (right) as function of time for a single causal (1Pre1Post10) stimulus. d, Left: depletion of vesicle pools (reserve and docked) induced by 30 pairing repetitions delivered at $5 \mathrm{~Hz}^{45}$ (Methods). The same vesicle depletion rule is applied to both glutamate and GABA. Right: BaP efficiency as function of time. BaP efficiency controls the axial resistance between soma and dendrite in order to phenomenologically capture the distance-dependent $\mathrm{BaP}$ attenuation ${ }^{46,47}$ (Methods). e, Activated enzyme concentration for CaM, CaN and CaMKII, as function of time for the stimulus 1Pre1Post10, 30 pairing repetitions delivered at $5 \mathrm{~Hz}$. Note that the vertical grey bar is the duration of the stimuli, $6 \mathrm{~s}$.

\section{Results}

A multi-timescale model of synaptic plasticity induction. We built a computational model of plasticity induction at a single CA3-CA1 rat glutamatergic synapse (Fig. 1). Our goal was to reproduce results on synaptic plasticity that explored the effects of several experimental parameters: fine timing differences between pre and postsynaptic spiking (Fig. 2-3); stimulation frequency (Fig. 4); animal age (Fig. 5); external calcium and magnesium (Fig. 6); stochasticity in the firing structure (Fig. 7), temperature and experimental conditions variations (Supplemental Information). Where possible, we set parameters to values previously estimated from experiments, and tuned the remainder within physiologically plausible ranges to reproduce our target plasticity experiments (Methods).

The model components are schematized in Fig. 1a (full details in Methods). For glutamate release, we used a twopool vesicle depletion and recycling system, which accounts for short-term presynaptic depression and facilitation. When glutamate is released from vesicles, it can bind to the postsynaptic $\alpha$-amino-3-hydroxy-5-methyl-4-isoxazolepropionic acid and $\mathrm{N}$-methyl-D-aspartate receptors (AMPArs and NMDArs, respectively). When dendritic spine voltage depolarises, it activates voltage-gated calcium channels (VGCCs) and removes magne- sium $\left(\mathrm{Mg}^{2+}\right)$ block from NMDArs. Backpropagating action potentials $(\mathrm{BaP})$ can also cause spine depolarisation. As an inhibitory component, we modelled a gamma-aminobutyric acid receptor (GABAr) synapse on the dendrite shaft ${ }^{48}$. Calcium ions influxing through VGCCs and NMDArs can activate hyperpolarising SK potassium channels ${ }^{49,50}$, bind to calmodulin (CaM) or to a generic calcium buffer. Calcium-bound calmodulin activates two major signalling molecules immediately downstream of

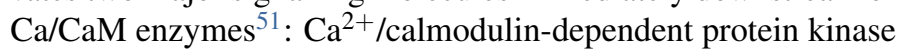
II (CaMKII) or calcineurin ( $\mathrm{CaN})$ phosphatase, also known as $\mathrm{PP}^{2} \mathrm{~B}^{52}$. We included these two proteins because CaMKII activation is necessary for Schaffer-collateral $\mathrm{LTP}^{53,54}$, while CaN activation is necessary for $\mathrm{LTD}^{55,56}$. Later, we show how we map the joint activity of CaMKII and CaN to LTP and LTD.

Synaptic receptors and ion channels have an inherent random behavior, stochastically switching between open and closed states $^{44}$. If the number of receptors or channels is large, then the variability of the total population activity becomes negligible relative to the mean ${ }^{57}$. However individual hippocampal synapses contain only small numbers of receptors and ion channels, for example $\sim 10$ NMDA receptors and $<15 \mathrm{VGCCs}^{58-60}$, making their total activation highly stochastic. Therefore, we modelled AMPAr, NMDAr, VGCCs and GABAr as stochastic processes. Presynaptic vesicle release events were also stochastic: glutamate 

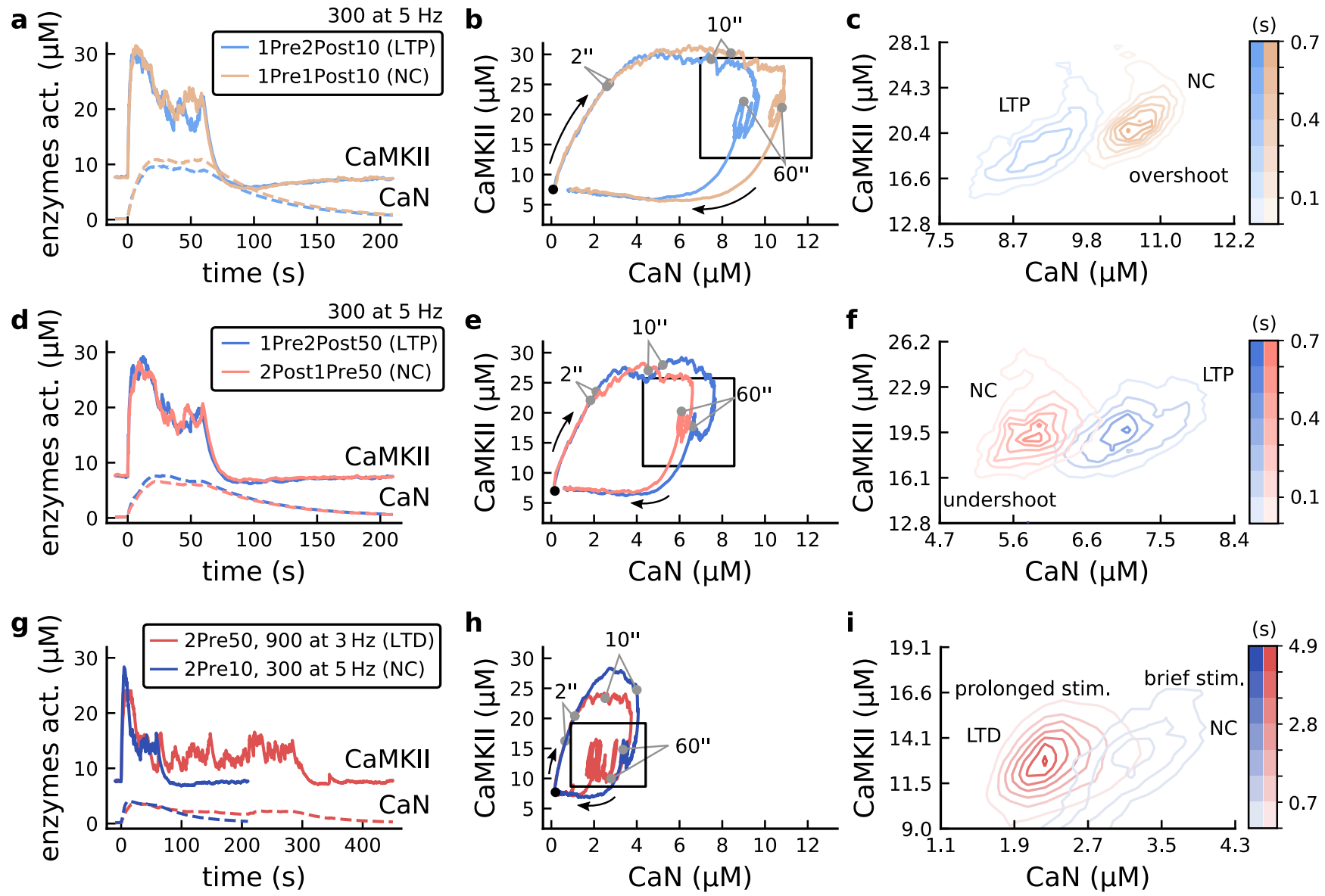

Fig. 2. I The duration and amplitude of the joint CaN-CaMKII activity differentiates plasticity protocols. a, Activity of CaMKII (solid line) and $\mathrm{CaN}$ (dashed line) $(\mu \mathrm{M})$ for two protocols. Experimentally the 1Pre2Post10 produces LTP, and 1Pre1Post10 produces no change (NC). Both are composed of 300 pairing repetitions delivered at $5 \mathrm{~Hz}$. b, Joint enzymatic activity (CaN-CaMKII) for the protocols in panel a. The black dot indicates the initial resting activity and the arrows the trajectory direction as function of time. The grey points mark the time position (x-axis in panel a) for both protocols at 2, 10 and $60 \mathrm{~s}$ (when the stimulation stops). The black square is the zoomed region in panel c. c, The mean time spent (colorbar) for each protocol in panel b (100 samples for each protocol for panelsc, $\mathbf{f}$ and $\mathbf{i})$. d, Same as in panel a, but for the LTP protocol, 1 Pre2Post50 and, the NC protocol, 2Post1Pre50. Both are composed of 300 pairing repetitions at $5 \mathrm{~Hz}$. e, Same as in panel $\mathbf{b}$ for protocols in panel $\mathbf{d}$. f, The mean time spent (colorbar) for each protocol in panel e. g, Same as in panel a and $\mathbf{d}$, but for two protocols with different frequencies and pulse repetitions. The LTD protocol, 2Pre50 900 at $3 \mathrm{~Hz}$ and, the NC protocol 2 Pre10 300 at $5 \mathrm{~Hz}$. h, Same as in panel b and e for protocols in panel $\mathbf{g}$. i, The mean time spent (colorbar) for each protocol in panel $\mathbf{h}$.

release was an all-or-nothing event, and the amplitude of each glutamate pulse was drawn randomly, modelling heterogeneity in vesicle size ${ }^{61}$. The inclusion of stochastic processes to account for an intrinsic noise in synaptic activation ${ }^{62}$ contrasts with most previous models in the literature, which either represent all variables as continuous and deterministic or add an external generic noise source ${ }^{63-65}$.

The synapse model showed nonlinear dynamics across multiple timescales. For illustration, we stimulated the synapse with single simultaneous glutamate and GABA vesicle releases (Fig. 1b). AMPArs and VGCCs open rapidly but close again within a few milliseconds. The dendritic GABAr closes more slowly, on a timescale of $\sim 10 \mathrm{~ms}$. NMDArs, the major calcium source, closes on timescales of $\sim 50 \mathrm{~ms}$ and $\sim 250 \mathrm{~ms}$ for the GluN2A and GluN2B subtypes, respectively.

To show the typical responses of the spine head voltage and $\mathrm{Ca}^{2+}$, we stimulated the synapse with a single presynaptic pulse (EPSP) paired $10 \mathrm{~ms}$ later with a single $\mathrm{BaP}$ (1Pre1Post10) (Fig. $1 \mathrm{c}$, left). For this pairing, when $\mathrm{BaP}$ is triggered immediately after an EPSP, it leads to a large $\mathrm{Ca}^{2+}$ transient aligned with the BaP due to the NMDArs first being bound by glutamate then unblocked by the $\mathrm{BaP}$ depolarisation (Fig. 1c, right).

Single pre or postsynaptic stimulation pulses did not cause depletion of vesicle reserves or substantial activation of the enzymes. To illustrate these slower-timescale processes, we stimulated the synapse with a prolonged protocol: one presynaptic pulse followed by one postsynaptic pulse $10 \mathrm{~ms}$ later, repeated 30 times at $5 \mathrm{~Hz}$ (Fig. 1d-e). The number of vesicles in both the docked and reserve pools decreased substantially over the course of the stimulation train (Fig. 1d left), which in turn causes decreased vesicle release probability. Similarly, by the 30th pulse, the dendritic BaP amplitude had attenuated to $\sim 85 \%$ ( $~ 70 \%$ BaP efficiency; Fig. $1 \mathrm{~d}$ right) of its initial amplitude, modelling the effects of slow dendritic sodium channel inactivation ${ }^{47,66}$. CaM concentration rose rapidly in response to calcium transients but also decayed back to baseline on a timescale of $\sim 500 \mathrm{~ms}$ (Fig. 1e top). In contrast, the concentration of active CaMKII and $\mathrm{CaN}$ accumulated over a timescale of seconds, reaching a sustained peak during the stimu- 
lation train, then decayed back to baseline on a timescale of $\sim 10$ and $\sim 120$ s respectively, in line with experimental data ${ }^{51,54,67}$ (Fig. 1e).

The effects of the stochastic variables can be seen in Fig. $1 \mathrm{~b}-\mathrm{d}$. The synaptic receptors and ion channels open and close randomly (Fig. 1b). Even though spine voltage, calcium, and downstream molecules were modelled as continuous and deterministic, they inherited some randomness from the upstream stochastic variables. As a result, there was substantial trial-totrial variability in the voltage and calcium responses to identical pre and postsynaptic spike trains (grey traces in Fig. 1d). This variability was also passed on to the downstream enzymes CaM, CaMKII and $\mathrm{CaN}$, but was filtered and therefore attenuated by the slow dynamics of CaMKII and CaN. In summary, the model contained stochastic nonlinear variables acting over five different orders of magnitude of timescale, from $\sim 1 \mathrm{~ms}$ to $\sim 1$ min, making it sensitive to both fast and slow components of input signals.

Distinguishing between stimulation protocols using the CaMKII and CaN joint response. It has proven difficult for simple models of synaptic plasticity to capture the underlying rules and explain why some stimulation protocols induce plasticity while others do not. We tested the model's sensitivity by simulating its response to a set of protocols used by ${ }^{24}$ in a recent ex vivo experimental study on adult (P50-55) rat hippocampus with blocked GABAr. We focused on three pairs of protocols (three rows in Fig. 2). In each case $i^{24}$ 's experiments, one of the pair induced LTP or LTD, while the other subtly different protocol caused no change (NC) in synaptic strength. We asked if the model's joint CaMKII-CaN activity could distinguish between each pair of protocols.

The first pair of protocols differed in intensity. A protocol which caused no plasticity consisted of 1 presynaptic spike followed $10 \mathrm{~ms}$ later by one postsynaptic spike repeated at $5 \mathrm{~Hz}$ for one minute (1Pre1Post10, 300 at $5 \mathrm{~Hz}$ ). The other protocol induced LTP, but differed only in that it included a postsynaptic doublet instead of a single spike (1Pre2Post10, 300 at $5 \mathrm{~Hz}$ ), implying a slightly stronger BaP amplitude initially. For the plots in Fig. 2a, it was not possible to set a single concentration threshold on either CaMKII or $\mathrm{CaN}$ that would discriminate between the protocols.

To achieve better separability, we combined the activity of the two enzymes, plotting the joint $\mathrm{CaMKII}$ and $\mathrm{CaN}$ responses against each other on a 2D plane (Fig. 2b). In this geometric plot, the two protocol's trajectories can be seen to overlap for the initial part of the transient, but then diverge. To quantify trial to trial variability, we also calculated contour maps showing the mean fraction of time the trajectories spent in each part of the plane during the stimulation (Fig. 2c). Importantly, both the trajectories and contour maps were substantially non-overlapping between the two protocols, implying that they can be separated based on the joint CaN-CaMKII activity. We found that the 1Pre2Post10 protocol leads to a weaker response in both CaMKII and $\mathrm{CaN}$, corresponding to the lower blue traces Fig. 2b. The decreased response to the doublet protocol was due to the enhanced attenuation of dendritic BaP amplitude over the course of the simulation ${ }^{47}$, leading to less calcium influx through NMDArs and VGCCs (data not shown).

The second pair of protocols we explored differed in sequenc- ing. We stimulated the synapse model with one causal (EPSP$\mathrm{BaP}$ ) protocol involving a single presynaptic spike followed 50 ms later by a doublet of postsynaptic spikes (1Pre2Post50, 300 at $5 \mathrm{~Hz}$ ), repeated at $5 \mathrm{~Hz}$ for one minute, which ${ }^{24}$ found caused LTP. The other anticausal protocol involved the same total number of pre and postsynaptic spikes, but with the pre-post order reversed (2Post1Pre50, 300 at 5Hz). Experimentally this anticausal (BaP-EPSP) protocol did not induce plasticity. Notably, the only difference was the sequencing of whether the pre or postsynaptic neuron fired first, over a short time gap of $50 \mathrm{~ms}$. Despite the activations being apparently difficult to distinguish (Fig. 2d), we found that the LTP-inducing protocol caused greater CaN activation than the protocol that did not trigger plasticity. Indeed, this translated to a horizontal offset in both the trajectory and contour map (Fig. 2e-f), demonstrating that another pair of protocols can be separated in the joint CaN-CaMKII plane.

The third pair of protocols differed in both duration and intensity. In line with previous studies ${ }^{24}$ found that a train of doublets of presynaptic spikes separated by $50 \mathrm{~ms}$ repeated at a low frequency of $3 \mathrm{~Hz}$ for 5 minutes (2Pre50, 900 at $3 \mathrm{~Hz}$ ) induced LTD, while a slightly more intense but shorter duration protocol of presynaptic spike doublets separated by $10 \mathrm{~ms}$ repeated at $5 \mathrm{~Hz}$ for one minute $(2 \mathrm{Pre} 10,300$ at $5 \mathrm{~Hz}$ ) did not cause plasticity. When we simulated both protocols in the model (Fig. $2 \mathrm{~g}-\mathrm{i}$ ), both caused similar initial responses in CaMKII and CaN. In the shorter protocol, this activation decayed to baseline within $100 \mathrm{~s}$ of the end of the stimulation. However the slower and longer-duration $2 \mathrm{Pre} 503 \mathrm{~Hz} 900 \mathrm{p}$ protocol caused an additional sustained, stochastically fluctuating, plateau of activation of both enzymes (Fig. 2g). This resulted in the LTD-inducing protocol having a downward and leftward-shifted CaN-CaMKII trajectory and contour plot, relative to the other protocol (Fig. 2h-i). These results again showed that the joint $\mathrm{CaN}-\mathrm{CaMKII}$ activity may be useful to predict plasticity changes.

\section{A geometrical readout mapping joint enzymatic activity to} plasticity outcomes. We found that the simulated CaN-CaMKII trajectories from the two LTP-inducing protocols (1Pre2Post10 and 1 Pre2Post50, at Fig. 2a and D respectively) spent a large fraction of time near $\sim 20 \mu \mathrm{M}$ CaMKII and 7-10 $\mu \mathrm{M}$ CaN. In contrast, protocols that failed to trigger LTP had either lower (2Post1Pre50 and 2Pre10, Fig. 2d and 2g respectively), or higher CaMKII and CaN activation (1Pre1Post10, Fig. 2a). The LTDinducing protocol, by comparison, spent a longer period in a region of sustained but lower $\sim 12 \mu \mathrm{M} \mathrm{CaMKII}$ and $\sim 2 \mu \mathrm{M} \mathrm{CaN}$ and activation. The plots in Fig. $2 \mathrm{c}, \mathrm{f}$ and g, show contour maps of histograms of the joint CaMKII-CaN activity, indicating where in the plane the trajectories spent most time. Fig. $\mathrm{c}$ and $\mathrm{f}$ indicate this measure can be used to predict plasticity, because the $\mathrm{NC}$ and LTP protocol histograms are largely non-overlapping. In Fig. 2c, the NC protocol response 'overshoots' the LTP protocol response, whereas in Fig. $2 \mathrm{f}$ the NC protocol response 'undershoots' the LTP protocol response. In contrast, when we compared the response histograms for LTD and NC protocols, we found a greater overlap (Fig. 2i). This suggested that in this case the histogram alone was not sufficient to separate the protocols, and that protocol duration is also important. LTD induction (2Pre50) required a more prolonged activation than NC (2Pre10).

To design a geometrical readout mechanism to map the 
bioRxiv preprint doi: https://doi.org/10.1101/2021.03.30.437703; this version posted May 27, 2021. The copyright holder for this preprint (which was not certified by peer review) is the author/funder, who has granted bioRxiv a license to display the preprint in perpetuity. It is made available under aCC-BY-NC-ND 4.0 International license.

a

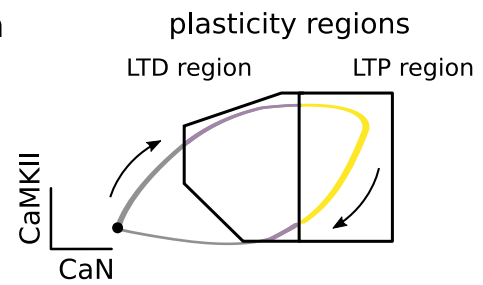

d
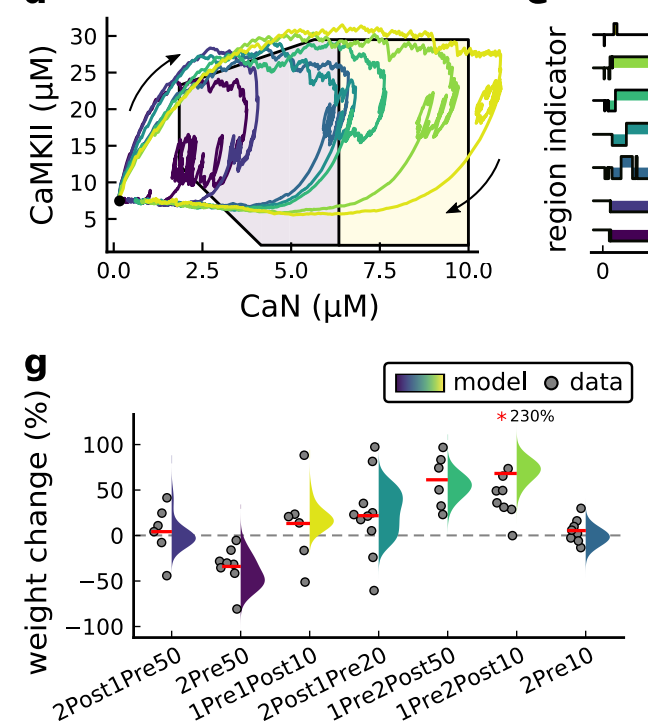

b

-
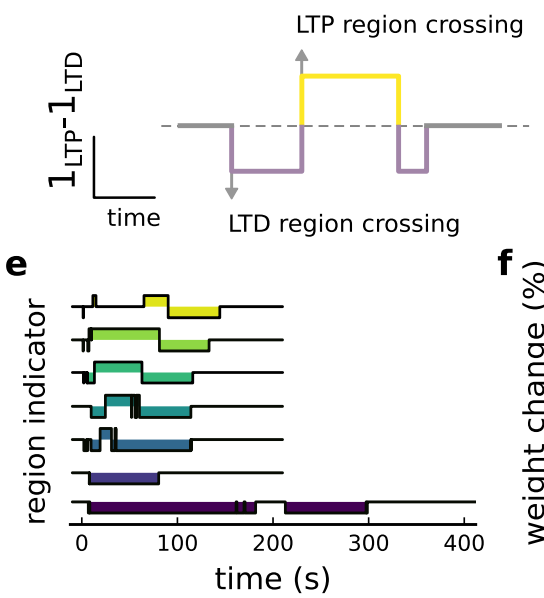

$\mathbf{h}$

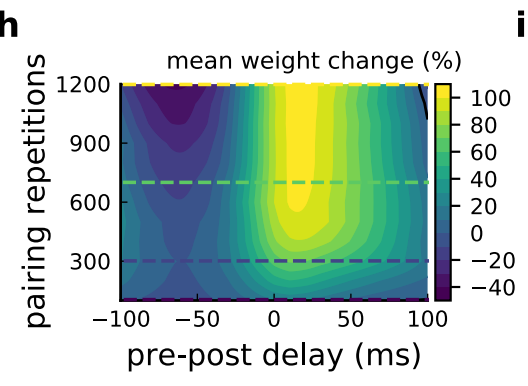

C plasticity Markov chain

LTP transitions (fast rate)

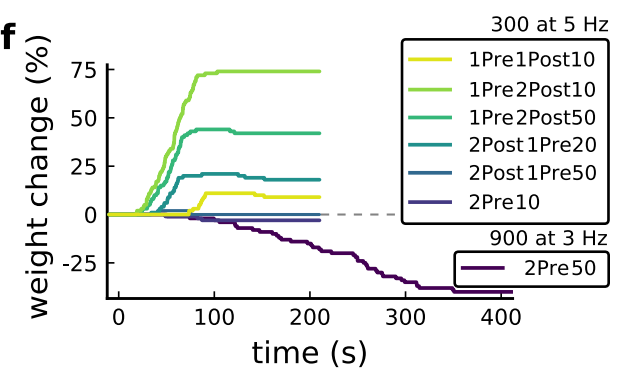

i 1 Pre2Post(delay) pairings at $5 \mathrm{~Hz}$

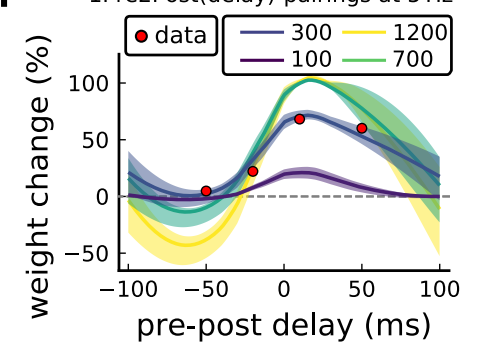

Fig. 3. I Read-out strategy an Tigaret et al. $\mathbf{2 0 1 6}^{24}$ experiment. a, Illustration of the joint CaMKII and CaN activities crossing the plasticity regions. Arrows indicate the flow of time, starting at the black dot. Note that here time is hidden and one can only see the changes in enzyme concentrations. b, Region indicator showing when $\mathrm{CaN}$ and CaMKII crosses the LTD or LTP regions in panel a. Leaving the region activates a leaking mechanism that keeps track of the accumulated time inside the region. Such leaking mechanism drives the transition rates used to predict plasticity (Methods). c, Plasticity Markov chain with three states: LTD, LTP and NC. There are only two transition rates which are functions of the plasticity region indicator (Methods). The LTP transition is fast whereas the transition LTD is slow, meaning that LTD change requires longer time inside the LTD region (panel a). The NC state starts with 100 processes. d, Joint CaMKII and CaN activity for all Tigaret protocols (labelled in F). The stimulus ends when the trajectory becomes smooth. Corresponds to Fig. 2b,e and h, at $60 \mathrm{~s}$. e, Region indicator for the protocols labelled in F. The upper square bumps are caused by the protocol crossing the LTP region, the lower square bumps when the protocol crosses the LTD region (as in panel d). f, Synaptic weight (\%) as function of time for each protocol. The weight change is defined as the number (out of 100) of states in the LTP state minus the number of states in the LTD state (panel c). The trajectories correspond to the median of the simulations in panel $\mathbf{g}$. $\mathbf{g}$, Synaptic weight change (\%) predicted by the model compared to data (EPSC amplitudes) from ${ }^{24}$ (100 samples for each protocol, also for panel $\mathbf{h}$ and $\mathbf{i}$ ). The data (grey dots) was provided by ${ }^{24}$ (note an $230 \%$ outlier as the red asterisk), red bands indicate data means. h, Predicted mean synaptic weight change (\%) as function of delay (ms) and number of pairing repetitions (pulses) for the protocol 1Pre2Post(delay), for delay between -100 and 100 . LTD is induced by 2 Post 1 Pre 50 after at least 500 pulses. The mean weight change along each dashed line is reported in the STDP curves in panel i. i, Synaptic weight change (\%) as function of pre-post delay. Each plot corresponds to a different pairing repetition number (legend). The solid line shows the mean, and the ribbons are the 2 nd and 4 th quantiles. The red dots are the data means estimated in ${ }^{24}$, also shown in panel $\mathbf{g}$.

enzyme activity to plasticity outcomes, we first drew nonoverlapping boxes of LTP and LTD "plasticity regions" in the CaN-CaMKII plane (Fig. 3a). We positioned these regions over the parts of the phase space where the enzyme activities corresponding to the LTP- and LTD-inducing protocols were most different, as shown by trajectories in Fig. 2. We then fixed these regions for all subsequent parts of this study. When a trajectory enters in one of these plasticity regions, it activates LTD or LTP indicator variables (Methods) which encode the joint enzyme activities (trajectories in the phase plots) transitions across the LTP and LTD regions over time (Fig. 3b). These indicator variable drove transition rates in a plasticity Markov chain used to predict LTP or LTD (Fig. 3c), see Methods. The LTD transition rates were slower than the LTP transition rates, to reflect studies showing that LTD requires sustained synaptic stimulation ${ }^{20-22}$. The parameters for this plasticity Markov chain (Methods) were fit to the plasticity induction outcomes from different protocols (Table M1). In the beginning of the simulation, the plasticity Markov chain starts with 100 processes ${ }^{48}$ in the state NC, with each variable representing $1 \%$ weight change, an abstract measure of synaptic strength that can be either EPSP, EPSC, or field EPSP slope depending on the experiment. Each process can transit stochastically between NC, LTP and LTD states. At the end of the protocol, the plasticity outcome is given by the difference between the number of processes in the LTP and the LTD states (Methods).

This readout mechanism acts as a parsimonious model of the complex signalling cascade linking CaMKII and $\mathrm{CaN}$ activation to expression of synaptic plasticity ${ }^{68}$. It can be considered as a two-dimensional extension of previous computational studies that applied analogous 1D threshold functions to dendritic spine calcium concentration $^{18,19,30,31}$. Our model is scalable, as it gives 
a

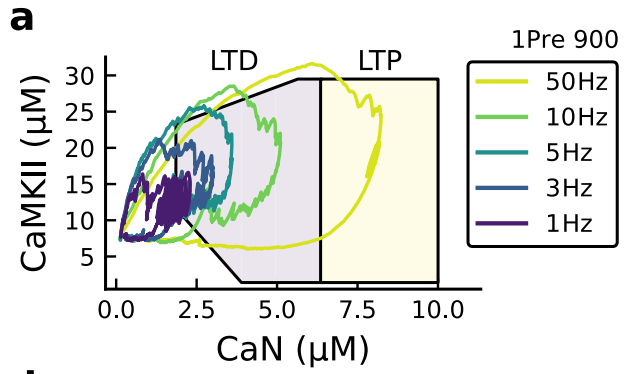

b

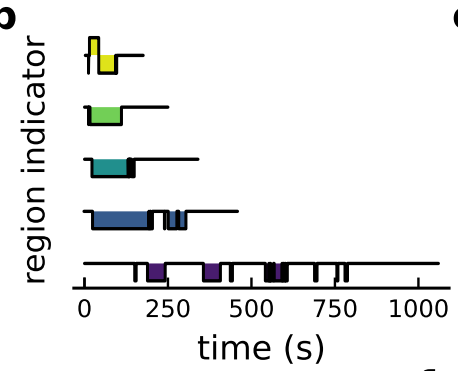

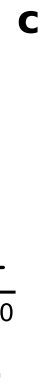
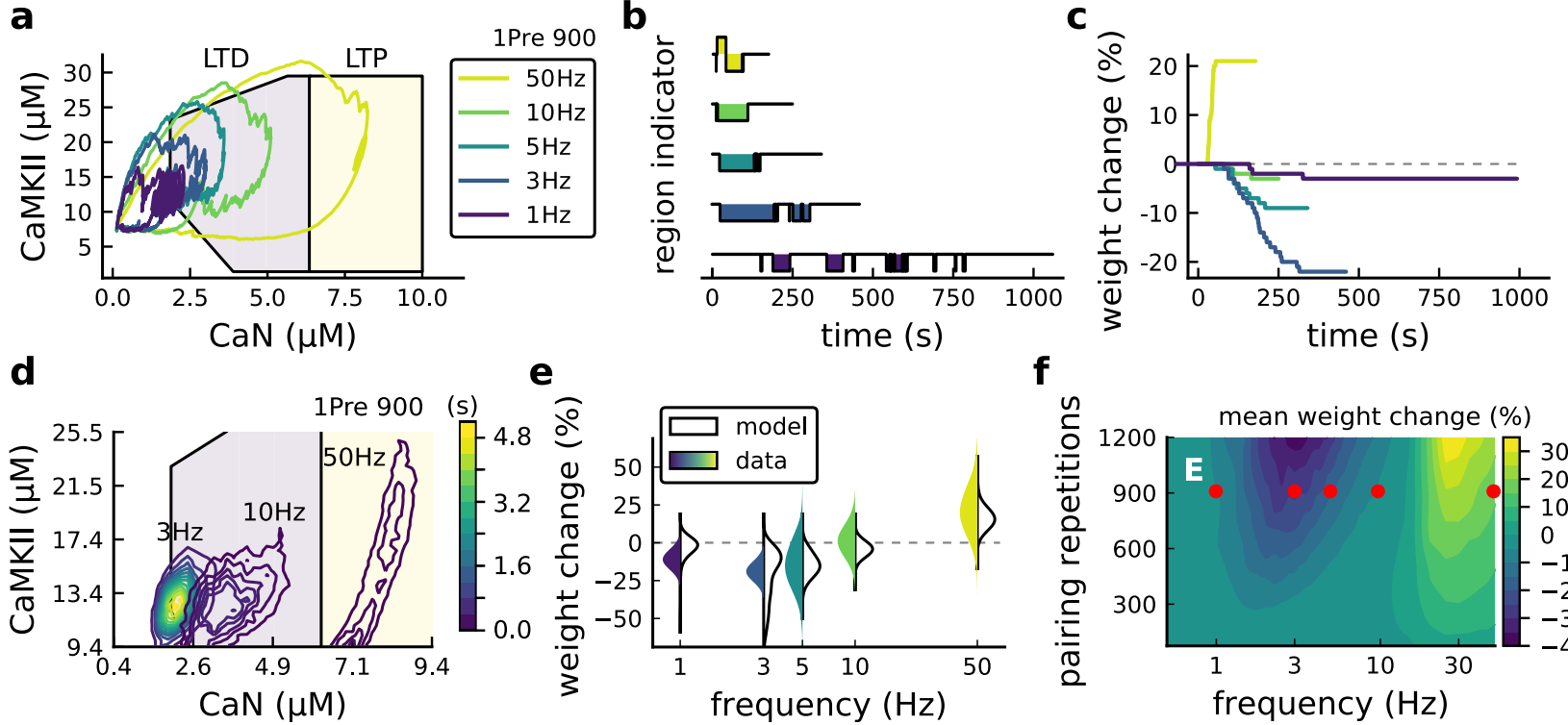

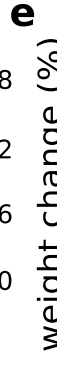
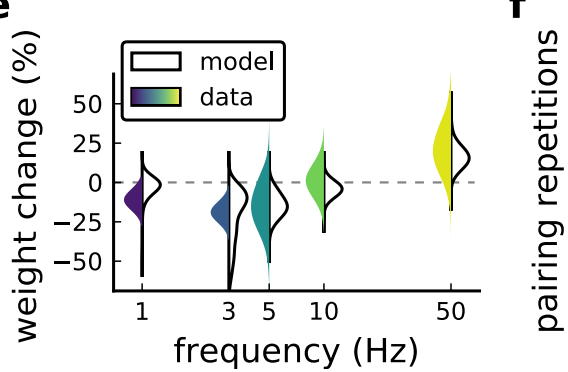

Fig. 4. I Frequency dependent plasticity, Dudek and Bear $1992{ }^{9}$ dataset. a, Example traces of joint CaMKII-CaN activity for each of ${ }^{9}$ 's protocols. b, Region indicator showing when the joint CaMKII-CaN activity crosses the LTD or LTP regions for each protocol in panel a. c, Synaptic weight change (\%) as function of time for each protocol, analogous to Fig. 3c. Trace colours correspond to panel a. The trajectories displayed were chosen to match the medians in panel e. d, Mean (100 samples) time spent (s) for protocols 1Pre for 900 pairing repetitions at 3, 10 and $50 \mathrm{~Hz}$. e, Comparison between data from ${ }^{9}$ and our model (1Pre 900p, 300 samples per frequency, Table M1). Data are represented as normal distributions with the mean and variance extracted from ${ }^{9}$. Note that data from Dudek and Bear are given in field EPSP slope change. f, Prediction for the mean weight change $(\%)$ varying the stimulation frequency and pulse number $\left(24 \times 38 \times 100\right.$ data points, respectively pulse $\mathrm{x}$ frequency $\mathrm{x}$ samples). The red dots show the ${ }^{9}$ protocol parameters, the corresponding results are shown in panel e.

the possibility for the readout to be extended to dynamics of $n$ different molecules, using $n$-dimensional closed regions.

In Fig. 3d, we plot the model's responses to seven different plasticity protocols used by ${ }^{24}$ by overlaying example CaMKII$\mathrm{CaN}$ trajectories for each protocol with the LTP and LTD regions. The corresponding region occupancies are plotted as function of time in E, and long-term alterations in the synaptic strength are plotted as function of time in F. The three protocols that induced LTP in the ${ }^{24}$ experiments spent substantial time in the LTP region, and so triggered potentiation. In contrast, the 1Pre1Post10 (yellow trace) overshoots both regions, crossing them only briefly on its return to baseline, and so resulted in little weight change. The protocol that induced LTD (2Pre50, purple trace) is five times longer than other protocols, spending sufficient time inside the LTD region (Fig. 3f). In contrast, two other protocols that spent time in the same LTD region of the CaN-CaMKII plane (2Post1Pre50 and 2Pre10) were too brief to induce LTD. These protocols were also not strong enough to reach the LTP region, so resulted in no net plasticity, again consistent with ${ }^{24}$ 's experiments.

We observed run-to-run variability in the amplitude of the predicted plasticity, due to the inherent stochasticity in the model. In Fig. 3g, we plot the distribution of the predicted plasticity from each protocol (colours) alongside the data from ${ }^{24}$ 's study, finding a good correspondence.

Experimentally, LTP can be induced by few pulses while LTD usually requires longer-duration stimulation ${ }^{20-22}$. We incorporated this effect into the readout model by letting LTP have faster transition rates than LTD (Fig. 3c). ${ }^{24}$ found that 300 repetitions of anticausal post-before-pre pairings did not cause LTD, in contrast to the canonical spike-timing-dependent plasticity curve ${ }^{7}$. We hypothesized that LTD might indeed appear with the anticausal ${ }^{24}$ protocol (Table M1) if stimulation duration was increased. To explore this possibility in the model, we systematically varied the number of paired repetitions from 100 to 1200, and also co-varied the pre-post delay from -100 to $100 \mathrm{~ms}$. Fig. 3h shows a contour plot of the predicted mean synaptic strength change across for the 1Pre2Post(delay) stimulation protocol for different numbers of pairing repetitions. A LTD window appears after $\sim 500$ pairing repetitions for some anticausal pairings, in line with our hypothesis. The magnitude of LTP also increases with pulse number, for causal positive pairings. For either 100 or 300 pairing repetitions, only LTP or NC is induced (Fig. 3i). The model also made other plasticity predictions by varying ${ }^{24}$ 's experimental conditions (Fig. S1). In summary, our model readout reveals that the direction and magnitude of the change in synaptic strength can be predicted from the joint CaMKII-CaN activity in the LTP and LTD regions.

Frequency-dependent plasticity. The stimulation protocols used by ${ }^{24}$ explored how subtle variations in pre and postsynaptic spike timing influenced the direction and magnitude of plasticity (see Table M1 for experimental differences). In contrast, traditional synaptic plasticity protocols exploring the role of presynaptic stimulation frequency did not measure the timing of co-occurring postsynaptic spikes ${ }^{9,69,70}$. These studies found that long-duration low-frequency stimulation induces LTD, whereas short-duration high-frequency stimulation induces LTP, with a cross-over point of zero change at intermediate stimulation frequencies. In addition to allowing us to explore frequencydependent plasticity (FDP), this stimulation paradigm also gives us further constraints for LTD in the model since in ${ }^{24}$, only one LTD case was available. For FDP, we focused on modelling the 
experiments from ${ }^{9}$, who stimulated Schaffer collateral projections to pyramidal CA1 neurons with 900 pulses in frequencies ranging from $1 \mathrm{~Hz}$ to $50 \mathrm{~Hz}$. In addition to presynaptic stimulation patterns, the experimental conditions differed from ${ }^{24}$ in two other aspects: animal age and control of postsynaptic spiking activity (see Table M1 legend). We incorporated both age-dependence and EPSP-evoked-BaPs (Methods). Importantly, the read-out mechanism mapping joint CaMKII-CaN activity to plasticity is the same for all experiments in this work.

Fig. 4a shows the joint CaMKII-CaN activity when we stimulated the model with 900 presynaptic spikes at 1, 3, 5, 10 and $50 \mathrm{~Hz}^{9}$. Higher stimulation frequencies drove stronger responses in both $\mathrm{CaN}$ and CaMKII activities (Fig. 4a). Fig. 4b,c show the corresponding plasticity region indicator for the LTP/LTD region threshold crossings and the synaptic strength change. From this set of five protocols, only the $50 \mathrm{~Hz}$ stimulation drove a response strong enough to reach the LTP region of the plane (Fig. 4a a,d). Although the remaining four protocols drove responses primarily in the LTD region, only the 3 and $5 \mathrm{~Hz}$ stimulations resulted in substantial LTD. The $1 \mathrm{~Hz}$ and $10 \mathrm{~Hz}$ stimulations resulted in negligible LTD, but for two distinct reasons. Although the $10 \mathrm{~Hz}$ protocol's joint CaMKII-CaN activity passed through the LTD region of the plane (Fig. 4a,d), it was too brief to activate the slow LTD mechanism built into the readout (Methods). The $1 \mathrm{~Hz}$ stimulation, on the other hand, was prolonged, but its response was mostly too weak to reach the LTD region, crossing the threshold only intermittently (Fig. 4b, bottom trace). Overall the model matched well the mean plasticity response found by Dudek and Bear (Fig. 4e), following a classic BCM-like curve as function of stimulation frequency ${ }^{71,72}$.

We then used the model to explore the stimulation space in more detail by varying the stimulation frequency from $0.5 \mathrm{~Hz}$ to $50 \mathrm{~Hz}$, and varying the number of presynaptic pulses from 50 to 1200. Fig. 4 f shows a contour map of the mean synaptic strength change (\%) in this 2D frequency-pulse number space. Under ${ }^{9}$ 's experimental conditions, we found that LTD induction required at least $\sim 300$ pulses, at frequencies between $1 \mathrm{~Hz}$ and $3 \mathrm{~Hz}$. In contrast, LTP could be induced using $\sim 50$ pulses at $\sim 20 \mathrm{~Hz}$ or greater. The contour map also showed that increasing the number of pulses (vertical axis in Fig. 4e) increases the magnitude of both LTP and LTD. This was paralleled by a widening of the LTD frequency range, whereas the LTP frequency threshold remained around $\sim 20 \mathrm{~Hz}$, independent of pulse number. The pulse dependence amplitude increase predicted in Fig. 4 is also valid for ${ }^{24}$ experiment shown in Fig. S1f.

Ex vivo experiments in ${ }^{9}$ were done at $35^{\circ} \mathrm{C}$. However, lower temperatures are more widely used ex vivo because they extend brain slice viability. We performed further simulations testing temperature modifications for ${ }^{9}$ 's experiment, finding that it had a strong effect on plasticity outcomes (Fig. S2d-f).

Variations in plasticity induction with developmental age. The rules for induction of LTP and LTD change during development ${ }^{33,35}$, so a given plasticity protocol can produce different outcomes when delivered to synapses from young animals versus mature animals. For example, when ${ }^{33}$ tested the effects of low-frequency stimulation $(1 \mathrm{~Hz})$ on CA3-CA1 synapses from rats of different ages, they found that the magnitude of LTD decreases steeply with age from P7 until becoming minimal in mature animals $>$ P35 (Fig. 5a, circles). Across the same age range, they found that a theta-burst stimulation protocol induced progressively greater LTP magnitude with developmental age (Fig. 5b, circles). Paralleling this, multiple properties of neurons change during development: the NMDAr switches its dominant subunit expression from GluN2B to GluN2 $\mathrm{A}^{73-75}$, the reversal potential of the receptor (GABAr) switches from depolarising to hyperpolarizing ${ }^{34,76,77}$, and the action potential backpropagates more efficiently with age ${ }^{46}$. These mechanisms have been proposed to underlie the developmental changes in synaptic plasticity rules because they are key regulators of synaptic calcium signalling ${ }^{34,46}$. However, their sufficiency and individual contributions to the age-related plasticity changes are unclear. We incorporated these mechanisms in the model (Methods) by parameterizing each of the three components to vary with the animal's postnatal age, to test if they could account for the age-dependent plasticity data.

We found that elaborating the model with age-dependent changes in NMDAr composition, GABAr reversal potential, and $\mathrm{BaP}$ efficiency, while keeping the same plasticity readout parameters, was sufficient to account for the developmental changes in LTD and LTP observed by ${ }^{33}$ (Fig. 5a,b). We then explored the model's response to protocols of various stimulation frequencies, from 0.5 to $50 \mathrm{~Hz}$, across ages from P5 to P80 (Fig. 5c,e). Fig. 5c shows the synaptic strength change as function of stimulation frequency for ages P15, P25, P35 and P45. The magnitude of LTD decreases with age, while the magnitude of LTP increases with age. Fig. 5e shows a contour plot of the same result, covering the age-frequency space.

The $1 \mathrm{~Hz}$ presynaptic stimulation protocol in ${ }^{33}$ did not induce LTD in adult animals ${ }^{9}$. We found that the joint CaN-CaMKII activity trajectories for this stimulation protocol underwent an age-dependent leftward shift beyond the LTD region (Fig. 5f). This implies that LTD is not induced in mature animals by this conventional LFS protocol due to insufficient activation of enzymes. In contrast, ${ }^{24}$ and $^{78}$ were able to induce LTD in adult rat tissue by combining LFS with presynaptic spike pairs repeated 900 times at $3 \mathrm{~Hz}$. Given these empirical findings and our modelling results, we hypothesized that LTD induction in adult animals requires that the stimulation protocol: 1) causes CaMKII and CaN activity to stay more in the LTD region than the LTP region, and 2) is sufficiently long to activate the LTD readout mechanism. With experimental parameters used by ${ }^{33}$, this may be as short as 300 pulses when multi-spike presynaptic protocols are used since the joint CaMKII-CaN activity can reach the LTD region more quickly than for single spike protocols. We simulated two such potential protocols as predictions: doublet and quadruplet spike groups delivered 300 times at $1 \mathrm{~Hz}$, with 50 $\mathrm{ms}$ between each pair of spikes in the group (Fig. 5d). The model predicted that both these protocols induce LTD in adults (green and blue curves), whereas as shown above, the single pulse protocol did not cause LTD (yellow curve). These findings suggest that the temporal requirements for inducing LTD may not be as prolonged as previously assumed, since they can be reduced by varying stimulation intensity. See Fig. S3 for frequency versus age maps for presynaptic bursts.

33 also performed theta-burst stimulation (TBS, Table M1) at different developmental ages, and found that LTP is not easily induced in young rats (Fig. 5b), see also ${ }^{35}$. The model qualitatively 
matches this trend, and also predicts that TBS induces maximal LTP around P21, before declining further during development (Fig. 5b, green curve). Similarly, we found that high-frequency stimulation induces LTP only for ages $>\mathrm{P} 15$, peaks at $\mathrm{P} 35$, then gradually declines at older ages (Fig. 5e). Note that in Fig. 5b, we used 6 epochs instead of 4 used by ${ }^{33}$ to increase LTP outcome which is known to washout after one hour for young rats ${ }^{35}$.

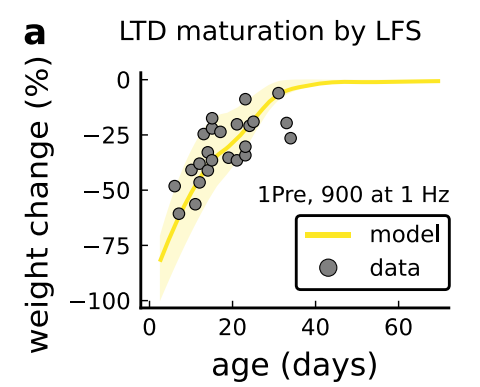

b LTP maturation by TBS

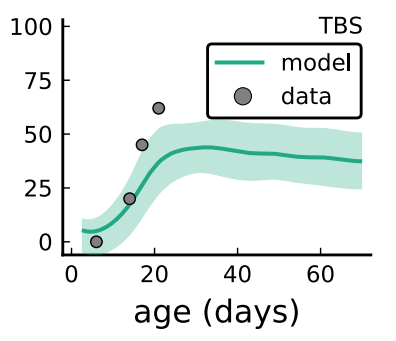

C age-dependent LTD/LTP shift

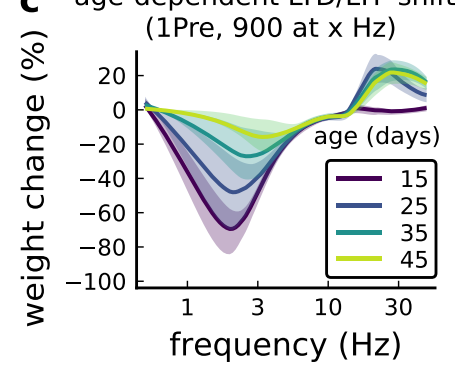

e
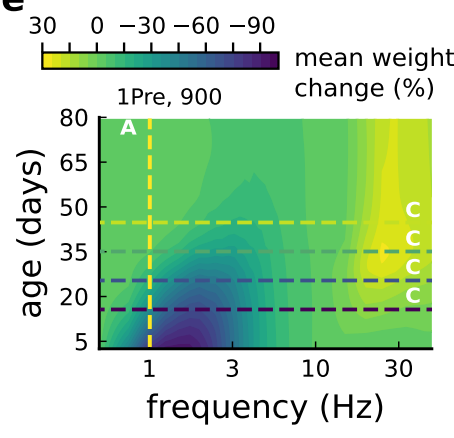

f

enhancing LTD in adulthood
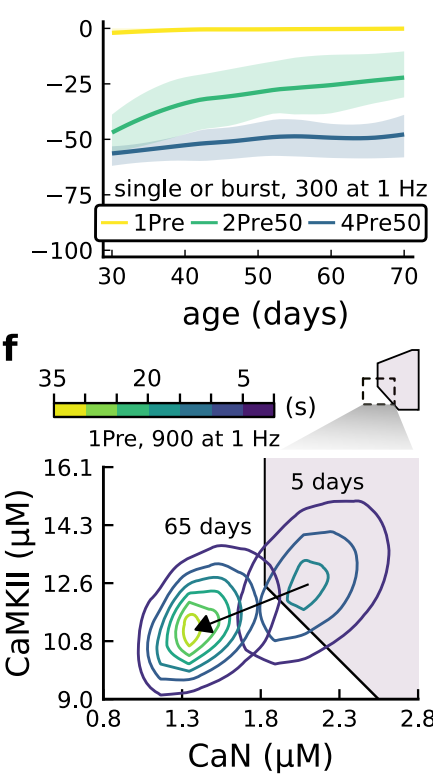

Fig. 5. I Age-dependent plasticity, Dudek and Bear $1993^{33}$ dataset a, Synaptic weight change for 1 Pre, 900 at $1 \mathrm{~Hz}$ as in ${ }^{33}$. The solid line is the mean and the ribbons are the 2 nd and 4 th quantiles predicted by our model (same for panel $\mathbf{b}, \mathbf{c}$ and $\mathbf{f}$ ). b, Synaptic weight change for Theta Burst Stimulation (TBS - 4Pre at $100 \mathrm{~Hz}$ repeated 10 times at $5 \mathrm{~Hz}$ given in 6 epochs at $0.1 \mathrm{~Hz}$ (Table M1) and ${ }^{33}$. c, Synaptic weight change as function of frequency for different ages. BCM-like curves showing that, during adulthood, the same LTD protocol becomes less efficient. It also shows that high-frequencies are inefficient at inducing LTP before P15. d, Synaptic weight change as function of age. Proposed protocol using presynaptic bursts to recover LTD at $\geq$ P35 with less pulses, 300 instead of the original 900 from $^{33}$. This effect is more pronounced for young rats. Fig. S3 shows a 900 pulses comparison. e, Mean synaptic strength change $(\%)$ as function of frequency and age for 1Pre 900 pulses $(32 \times 38 \times 100$, respectively, for frequency, age and samples). The protocols in ${ }^{33}$ (panel a) are marked with the yellow vertical line. The horizontal lines represent the experimental conditions of panel $\mathbf{c}$. Note the P35 was used for ${ }^{9}$ experiment in Fig. 4f. f, Mean time spent for the 1 Pre $1 \mathrm{~Hz} 900$ pulses protocol showing how the trajectories are left-shifted as rat age increases.

In contrast $\mathrm{to}^{33}$, $\mathrm{s}$ findings, other studies have found that
LTP can be induced in hippocampus in young animals $(<\mathrm{P} 15)$ with STDP. For example, ${ }^{34}$ found that at room temperature, 1Pre1Post10 induces LTP in young rats, whereas 1Pre2Post10 induces NC. This relationship was inverted for adults, with 1Pre1Post inducing no plasticity and 1 Pre2Post10 inducing LTP (Fig. S5).

Together, these results suggest that not only do the requirements for LTP/LTD change with age, but also that these agedependencies are different for different stimulation patterns. Finally, we explore which mechanisms are responsible for plasticity induction changes across development in the FDP protocol (Fig. S3) by fixing each parameter to young or adult values for the FDP paradigm. Our model analysis suggests that the NMDAr

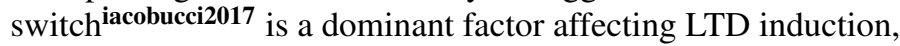
but the maturation of $\mathrm{BaP}^{46}$ is the dominant factor affecting LTP induction, with GABAr shift having only a weak influence on LTD induction for ${ }^{33}$ 's FDP.

Plasticity requirements during development do not necessarily follow the profile in ${ }^{33}$ as shown by ${ }^{34}$ 's STDP experiment. Our model shows that multiple developmental profiles are possible when experimental conditions vary within the same stimulation paradigm. This is illustrated in Fig. S5a-c by varying the age of STDP experiments done in different conditions.

Effects of extracellular calcium and magnesium concentration on plasticity. The canonical STDP rule ${ }^{7}$ measured in cultured neurons, high $\left[\mathrm{Ca}^{2+}\right]_{0}$, and at room temperature, was recently found not to be reproducible at physiological $\left[\mathrm{Ca}^{2+}\right]_{\mathrm{o}}$ in CA1 brain slices ${ }^{38}$. Instead, by varying the $\left[\mathrm{Ca}^{2+}\right]_{\mathrm{o}}$ and $\left[\mathrm{Mg}^{2+}\right]_{\mathrm{o}}$ they found a spectrum of STDP rules with either no plasticity or full-LTD for physiological $\left[\mathrm{Ca}^{2+}\right]_{\mathrm{o}}$ conditions $\left(\left[\mathrm{Ca}^{2+}\right]_{\mathrm{o}}<1.8\right.$ $\mathrm{mM})$ and a bidirectional rule for high $\left[\mathrm{Ca}^{2+}\right]_{\mathrm{o}}\left(\left[\mathrm{Ca}^{2+}\right]_{\mathrm{o}}>2.5\right.$ $\mathrm{mM}$ ), shown in Fig. 6a-c (data).

We attempted to reproduce ${ }^{38}$, s findings by varying $\left[\mathrm{Ca}^{2+}\right]_{\mathrm{o}}$ and $\left[\mathrm{Mg}^{2+}\right]_{0}$ with the following consequences for the model mechanisms (Methods). On the presynaptic side, $\left[\mathrm{Ca}^{2+}\right]_{\mathrm{o}}$ modulates vesicle release probability. On the postsynaptic side, high $\left[\mathrm{Ca}^{2+}\right]_{\mathrm{o}}$ reduces NMDAr conductance ${ }^{79}$, whereas $\left[\mathrm{Mg}^{2+}\right]_{\mathrm{o}}$ affects the NMDAr $\mathrm{Mg}^{2+}$ block ${ }^{80}$. Furthermore, spine $\left[\mathrm{Ca}^{2+}\right]_{0}$ influx activates SK channels, which hyperpolarize the membrane and indirectly modulate NMDAr activity ${ }^{50,81}$.

Fig. 6a-c compares our model to ${ }^{38}$ 's STDP data at different $\left[\mathrm{Ca}^{2+}\right]_{\mathrm{o}}$ and $\left[\mathrm{Mg}^{2+}\right]_{\mathrm{o}}$. Note that ${ }^{38}$ used $150(100)$ pairing repetitions for the anti-causal (causal) both delivered at $0.3 \mathrm{~Hz}$. At $\left[\mathrm{Ca}^{2+}\right]_{\mathrm{o}}=1.3 \mathrm{mM}$, Fig. 6a shows the STDP rule induced weak LTD for brief causal delays. At $\left[\mathrm{Ca}^{2+}\right]_{0}=1.8 \mathrm{mM}$, in Fig. 6 , the model predicted a full-LTD window. At $\left[\mathrm{Ca}^{2+}\right]_{0}=3 \mathrm{mM}$, in Fig. $6 c$, it predicted a bidirectional rule with a second LTD window for long pre-before-post pairings, previously theorized by ${ }^{26}$.

Fig. 6d illustrates the time spent by the joint CaN-CaMKII activity for 1 Pre1Post 10 using ${ }^{38}$ 's experimental conditions. Each density plot corresponds to a different panel in Fig. 6 with the respective $\mathrm{Ca} / \mathrm{Mg}$. The response under low $\left[\mathrm{Ca}^{2+}\right]_{\mathrm{o}}$ spent most time inside the LTD region, but high $\left[\mathrm{Ca}^{2+}\right]_{0}$ shifts the trajectory to the LTP region. Fig. S4a presents density plots for the anticausal post-before-pre protocols.

${ }^{38}$ fixed the $\mathrm{Ca} / \mathrm{Mg}$ ratio at 1.5 , although aCSF formulations in the literature differ (see Table M1). Fig. S4d shows that varying $\mathrm{Ca} / \mathrm{Mg}$ ratio and $\left[\mathrm{Ca}^{2+}\right]_{\mathrm{o}}$ for ${ }^{38}$, $\mathrm{s}$ experiments restrict LTP to 

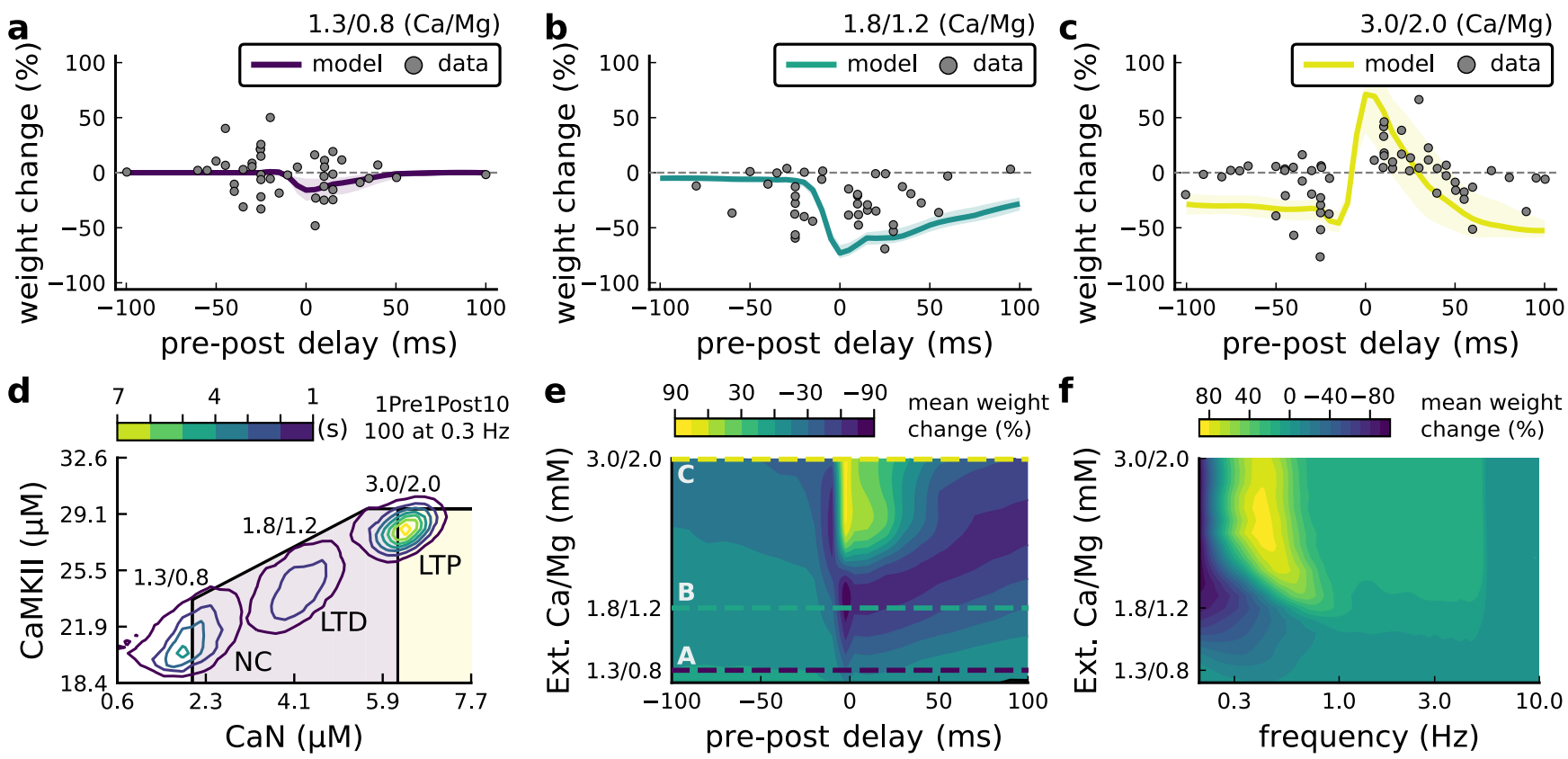

Fig. 6. Effects of extracellular calcium and magnesium concentrations on plasticity a, Synaptic weight (\%) for a STDP rule with $\left[\mathrm{Ca}^{2+}\right]_{o}=1.3 \mathrm{mM}$ (fixed ratio, $\mathrm{Ca} / \mathrm{Mg}=1.5$ ). According to the data extracted from ${ }^{38}$, the number of pairing repetitions for positive (negative) delays is $100(150)$, both delivered at $0.3 \mathrm{~Hz}^{38}$. The solid line is the mean, and the ribbons are the 2nd and 4th quantiles predicted by our model (all panels use 100 samples). b, Same as A, but for $\left[\mathrm{Ca}^{2+}\right]_{\mathrm{o}}=1.8 \mathrm{mM}(\mathrm{Ca} / \mathrm{Mg}$ ratio $=1.5)$. c, Same as A, but for $\left[\mathrm{Ca}{ }^{2+}\right]_{\mathrm{o}}=3 \mathrm{mM}(\mathrm{Ca} / \mathrm{Mg}$ ratio $=1.5)$. d, Mean time spent for causal pairing, 1 Pre1Post10, at different $\mathrm{Ca} / \mathrm{Mg}$ concentrations. The contour plots are associated with the panels $\mathbf{a}$, b and $\mathbf{c}$. e, STDP and extracellular $\mathrm{Ca} / \mathrm{Mg}$. Synaptic weight change (\%) for causal (1Pre1Post10, $100 \mathrm{at} 0.3 \mathrm{~Hz}$ ) and anticausal (1Post1Pre10, 150 at $0.3 \mathrm{~Hz}$ ) pairings varying extracellular $\mathrm{Ca}$ from 1.0 to $3 \mathrm{mM}(\mathrm{Ca} / \mathrm{Mg}$ ratio $=1.5)$. The dashed lines represent the experiments in the panel $\mathbf{a}, \mathbf{b}$ and $\mathbf{c}$. We used $21 \times 22 \times 100$ data points, respectively calcium x delay x samples. f, Varying frequency and extracellular $\mathrm{Ca} / \mathrm{Mg}$ for the causal pairing 1 Pre1Post 10 , 100 at $0.3 \mathrm{~Hz}$. Contour plot showing the mean synaptic weight (\%) for a single causal pairing protocol (1Pre1Post10, 100 samples) varying frequency from 0.1 to $10 \mathrm{~Hz}$ and $\left[\mathrm{Ca}^{2+}\right]_{0}$ from 1.0 to $3 \mathrm{mM}(\mathrm{Ca} / \mathrm{Mg}$ ratio $=1.5)$. We used $21 \times 18 \times 100$ data points, respectively calcium $\mathrm{x}$ frequency $\mathrm{x}$ samples.

$\mathrm{Ca} / \mathrm{Mg}>1.5$ and $\left[\mathrm{Ca}^{2+}\right]_{\mathrm{o}}>1.8 \mathrm{mM}$.

Our model can also identify the transitions between LTD and LTP depending on $\mathrm{Ca} / \mathrm{Mg}$. Fig. 6e shows a map of plasticity as function of pre-post delay and $\mathrm{Ca} / \mathrm{Mg}$ concentrations and the parameters where LTP is induced for the 1Pre1Post10 protocol. Since plasticity rises steeply at around $\left[\mathrm{Ca}^{2+}\right]_{0}=2.2 \mathrm{mM}$, small fluctuations in $\left[\mathrm{Ca}^{2+}\right]_{\mathrm{o}}$ near this boundary could cause qualitative transitions in plasticity outcomes. For anticausal pairings, increasing $\left[\mathrm{Ca}^{2+}\right]_{\mathrm{o}}$ increases the magnitude of LTD (Fig. S4b illustrates this with ${ }^{38}$, s data).

${ }^{38}$ also found that increasing the pairing frequency to 5 or 10 $\mathrm{Hz}$ results in a transition from LTD to LTP for 1Pre1Post10 at $\left[\mathrm{Ca}^{2+}\right]_{0}=1.8 \mathrm{mM}$ (Fig. S4c), similar frequency-STDP behaviour has been reported in cortex ${ }^{10}$. In Fig. 6f, we varied both the pairing frequencies and $\left[\mathrm{Ca}^{2+}\right]_{0}$ and we observe similar transitions to ${ }^{38}$. However, the model's transition for $\left[\mathrm{Ca}^{2+}\right]_{0}=1.8 \mathrm{mM}$ was centred around $0.5 \mathrm{~Hz}$, which was untested by ${ }^{38}$. The model predicted no plasticity at higher frequencies, unlike the data, that shows scattered LTP and LTD (see Fig. S4c). Fig. S1d and S4e shows that ${ }^{24}$ 's burst-STDP and ${ }^{38}$ 's STDP share a similar transition structure, but not ${ }^{9}$ 's FDP.

In contrast to ${ }^{38}$, s results, we found that setting low $\left[\mathrm{Ca}^{2+}\right]_{\mathrm{o}}$ for ${ }^{24}$ 's burst-STDP abolishes LTP, and does not induce strong LTD (Fig. S1d). For ${ }^{9}$ 's experiment, Fig. S2a $\left[\mathrm{Mg}^{2+}\right]_{0}$ controls a sliding threshold between LTD and LTP but not $\left[\mathrm{Ca}^{2+}\right]_{\mathrm{o}}$ (Fig. S2b). For another direct stimulation experiment, Fig. S4c shows that in an $\mathrm{Mg}$-free medium, LTP expression requires fewer pulses $^{21}$.

Despite exploring physiological $\left[\mathrm{Ca}^{2+}\right]_{\mathrm{o}}$ and $\left[\mathrm{Mg}^{2+}\right]_{\mathrm{o}}{ }^{38}$ use a non-physiological temperature $\left(30^{\circ} \mathrm{C}\right)$ which extends T-type VGCC closing times and modifies the CaN-CaMKII baseline (Fig. S5i). Fig. S5g,h shows comparable simulations for physiological temperatures. Overall our model predicts that temperature can change STDP rules in a similar fashion to $\left[\mathrm{Ca}^{2+}\right]_{0}$ (Fig. S4a,b). In summary, plasticity is highly sensitive to variations in extracellular calcium, magnesium, and temperature (Fig. S1a, Fig. S $\left.5 \mathbf{d}-\mathbf{f} ;{ }^{40}\right)$.

In vivo-like spike variability affects plasticity. In the above sections, we used highly regular and stereotypical stimulation protocols to replicate typical ex vivo plasticity experiments. In contrast, neural spiking in hippocampus in vivo is irregular and variable $^{78,82}$. It is unclear how natural firing variability affects the rules of plasticity induction ${ }^{27,83-85}$. We explored this question using model simulations by adding three distinct types of variability: 1) spike time jitter, 2) failures induced by dropping spikes, 3) independent pre and postsynaptic Poisson spike trains ${ }^{83}$.

We introduced spike timing jitter by adding zero-mean Gaussian noise (s.d. $\sigma$ ) to pre and postsynaptic spikes, changing spike pairs inter-stimulus interval (ISI). In Fig. 7a, we plot the LTP magnitude as function of jitter magnitude (controlled by $\sigma$ ) for protocols taken from ${ }^{24}$. With no jitter, $\sigma=0$, these protocols have different LTP magnitudes (corresponding to Fig. 3) and become similar once $\sigma$ increases. The three protocols with a postsynaptic spike doublet gave identical plasticity for $\sigma=50$ 
ms.

To understand the effects of jittering, we plotted the trajectories of CaN-CaMKII activity (Fig. 7c). 2Post1Pre50 which "undershoots" the LTP region shifted into the LTP region for jitter $\sigma=50 \mathrm{~ms}$. In contrast, 1Pre1Post10 which "overshoots" the LTP region shifted to the opposite direction towards the LTP region.

Why does jitter cause different spike timing protocols to yield similar plasticity magnitudes? Increasing jitter causes a fraction of pairings to invert causality. Therefore, the jittered protocols became a mixture of causal and anticausal pairings (Fig. 7c). This situation occurs for all paired protocols. So any protocol with the same number spikes will become mixed if the jitter is large enough. Note that despite noise the mean frequency was conserved at $5 \pm 13.5 \mathrm{~Hz}$ (see Fig. 7e).

Next, we studied the effect of spike removal. In the previous sections, synaptic release probability was $\sim 60 \%$ (for $\left[\mathrm{Ca}^{2+}\right]_{\mathrm{o}}=$ $2.5 \mathrm{mM}$ ) or lower, depending on the availability of docked vesicles (Methods). However, baseline presynaptic vesicle release probability is heterogeneous across CA3-CA1 synapses, ranging from $\sim 10-90 \%{ }^{86,87}$ and likely lower on average in vivo ${ }^{88,89}$. BaPs are also heterogeneous with random attenuation profiles ${ }^{47}$ and spike failures ${ }^{90}$. To test the effects of pre and postsynaptic failures on plasticity induction, we performed simulations where we randomly removed spikes, altering the previously regular attenuation in ${ }^{24}$ 's protocols.

In Fig. 7b we plot the plasticity magnitude as function of sparsity (percentage of removed spikes). The sparsity had different specific effects for each protocol. 1Pre2Post10 and 1Pre2Post50 which originally produced substantial LTP were robust to spike removal until $\sim 60 \%$ sparsity. In contrast, the plasticity magnitude from both 1 Pre1Post10 and 2Post1Pre50 showed a nonmonotonic dependence on sparsity, first increasing then decreasing, with maximal LTP at $\sim 40 \%$ sparsity.

To understand how sparsity causes this non-monotonic effect on plasticity magnitude, we plotted the histograms of time spent in the CaN-CaMKII plane for 2Post1Pre50 for three levels of sparsity: $0 \%, 30 \%$ and $80 \%$ (Fig. 7d). For $0 \%$ sparsity, the activation spent most time at the border between the LTP and LTD regions, resulting in no plasticity. Increasing sparsity to $30 \%$ caused the activation to shift rightward into the LTP region because there was less attenuation of pre and postsynaptic resources. In contrast, at $80 \%$ sparsity, the activation moved into the LTD region because there were not enough events to substantially activate CaMKII and CaN. Since LTD is a slow process and the protocol duration is short (60s), there was no net plasticity. Therefore for this protocol, high and low sparsity caused no plasticity for distinct reasons, whereas intermediate sparsity enabled LTP by balancing resource depletion with enzyme activation.

Next we tested the interaction of jitter and spike removal. Fig. $7 \mathrm{f}$ shows a contour map of weight change as function of jitter and sparsity for the 2Post1Pre50 protocol, which originally induced no plasticity (Fig. 2). Increasing spike jitter enlarged the range of sparsity inducing LTP. In summary, these simulations (Fig. 7a,b,f and h) show that different STDP protocols have different degrees of sensitivity to noise in the firing structure, suggesting that simple plasticity rules derived from regular ex vivo experiments may not predict plasticity in vivo.

How does random spike timing affect rate-dependent plasticity? We stimulated the model with pre and postsynaptic Poisson
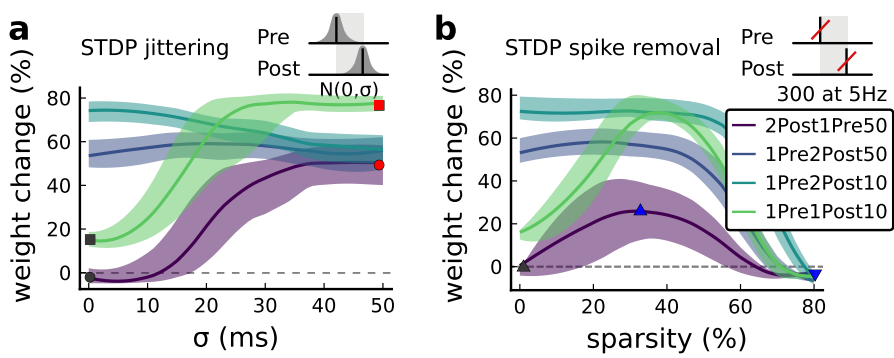

C convergence of causal and anti-causal mixtures

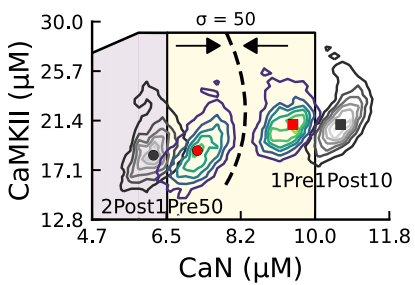

d spike removal prevents

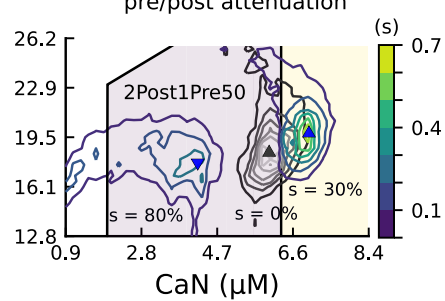

e

spike-timing mixture induced
by jittering

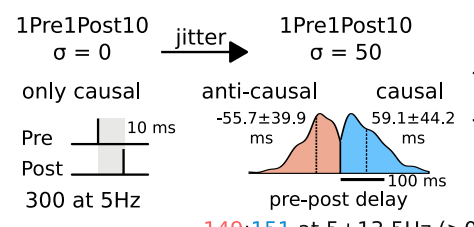

f 2 Post1Pre 50,300 at $5 \mathrm{~Hz}$ (spike removal + jitter) mean weight change $(\%)$
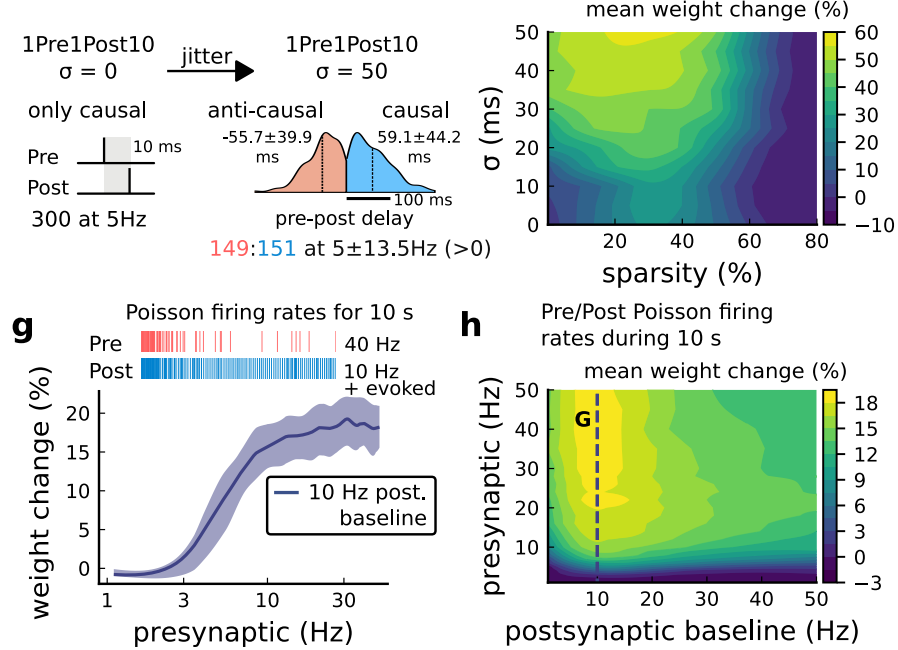

h

Pre/Post Poisson firing rates during $10 \mathrm{~s}$

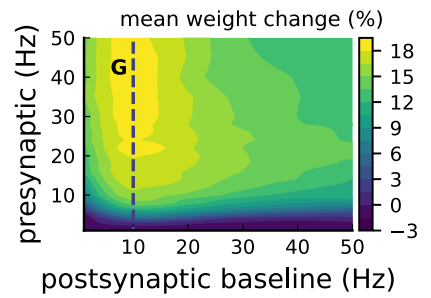

Fig. 7. I Jitter and spike dropping effects on STDP; and Poisson spike trains. a, Mean weight (\%) for the jittered STDP protocols. The solid line is the mean, and the ribbons are the 2nd and 4th quantiles predicted by our model estimated using 100 samples (same for all panels). b, Mean weight (\%) for the same ${ }^{24}$ protocols used at panel a subjected to random spike removal (sparsity \%). c, Mean time spent (s) varying jittering. Contour plot shows 2Post1Pre50 and 1Pre1Post10 (300 at 5 $\mathrm{Hz}$ ) with (grey contour plot) and with jittering (coloured contour plot) The circles and squares correspond to the marks in panel a. d, Mean time spent (s) varying sparsity. Contour plot in grey showing $0 \%$ sparsity for 2 Post1Pre 50300 at $5 \mathrm{~Hz}$ (see Fig. 2f). The contour plots show the protocol with spike removal sparsities at $0 \%$ (NC) $30 \%$ (LTP), and $80 \%$ (NC). The triangles correspond to the same marks in panel a. e, Distribution of the $50 \mathrm{~ms}$ jittering applied to the causal protocol 1Pre1Post10, 300 at $5 \mathrm{~Hz}$ in which nearly half of the pairs turned into anticausal The mean frequency is $5 \pm 13.5 \mathrm{~Hz}$. The protocol 2 Post1Pre 50 will have nearly half of the pairings turning into causal, making them have a similar firing structure and position inside the LTP region. f, Mean weight change (\%) combining both sparsity (panel b) and jittering (panel a) for 2 Post1Pre 50, 300 at $5 \mathrm{~Hz}$. g, Mean weight change (\%) of pre and postsynaptic Poisson spike train delivered simultaneously for $10 \mathrm{~s}$. The plot shows the plasticity outcome for different presynaptic firing rate (1000/frequency) for a fixed postsynaptic baseline at $10 \mathrm{~Hz}$. The upper raster plot depicts the released vesicles at $40 \mathrm{~Hz}$ and the postsynaptic baseline at $10 \mathrm{~Hz}$ (including the AP evoked by EPSP). h), Mean weight change $(\%)$ varying the rate of pre and postsynaptic Poisson spike train delivered simultaneously for $10 \mathrm{~s}$. The dashed line depicts panel g. 
spike trains for $10 \mathrm{~s}$, under ${ }^{9}$ 's experimental conditions. We systematically varied both the pre and postsynaptic rates (Fig. 7h). The 10s stimulation protocols induced only LTP, since LTD requires a prolonged stimulation ${ }^{21}$. LTP magnitude monotonically increased with the presynaptic rate (Fig. $7 \mathrm{~g}, \mathrm{~h}$ ). In contrast, LTP magnitude varied non-monotonically as function of postsynaptic rate, initially increasing until a peak at $10 \mathrm{~Hz}$, then decreasing with higher stimulation frequencies. This non-monotonic dependence on post-synaptic rate is inconsistent with classic rate-based models of Hebbian plasticity. We also investigated how this plasticity dependence on pre- and post-synaptic Poisson firing rates varies with developmental age (Fig. S2g-i). We found that at P5 no plasticity is induced, at P15 a LTP region appears at around 1 $\mathrm{Hz}$ postsynaptic rate, and at $\mathrm{P} 20$ plasticity becomes similar to the mature age, with a peak in LTP magnitude at $10 \mathrm{~Hz}$ postsynaptic rate.

\section{Discussion}

We built a model of a rat CA3-CA1 hippocampal synapse, including key electrical and biochemical components underlying synaptic plasticity induction (Fig. 1). We used a novel geometric readout of CaN-CaMKII dynamics (Fig. 2-4) to predict the outcomes from a range plasticity experiments with heterogeneous conditions: animal developmental age (Fig. 5), aCSF composition (Fig. 6), temperature (Supplemental files), and in vivo-like firing variability (Fig. 7).

Our model included critical components for plasticity induction at CA3-CA1 synapses: those affecting dendritic spine voltage, calcium signalling, and enzymatic activation. We were able to use model to make quantitative predictions, because its variables and parameters corresponded to biological components. This property allowed us to incorporate the model components' dependence on developmental age, external $\mathrm{Ca} / \mathrm{Mg}$ levels, and temperature to replicate datasets across a range of experimental conditions. The model is relatively fast to simulate, taking $\sim 1$ minute of CPU time to run 1 minute of biological time. These practical benefits should enable future studies to make experimental predictions on dendritic integration of multiple synaptic inputs ${ }^{42,91,92}$ and on the effects of synaptic molecular alterations in pathological conditions. In contrast, abstract models based on spike timing ${ }^{28,93,94}$ or simplified calcium dynamics ${ }^{18,30}$ must rely on ad hoc adjustment of parameters with less biological interpretability.

The model was built based the new concept that the full temporal activity of CaN-CaMKII over the stimulus duration ${ }^{51}$, and not their instantaneous activity levels ${ }^{18,19}$, is responsible for plasticity changes. We instantiated this concept by analyzing the joint CaN-CaMKII activity in the two-dimensional plane and designing polygonal plasticity readout regions (Fig. 3a). In doing so, we generalised previous work with plasticity induction based on single threshold and a slow variable ${ }^{26,28,30,95}$. Here, we used a two-dimensional readout, but anticipate a straightforward generalisation to higher-dimensions for different cellular processes in neuroscience but also in systems biology more broadly. The central discovery is that these trajectories, despite being stochastic, can be separated in the plane as function of the stimulus (Fig. 3). This is the basis of our new synaptic plasticity rule.

Let us describe the intuition behind our model more concisely.
First, we abstracted away the sophisticated cascade of plasticity expression. Second, the plasticity regions, crossed by the trajectories, are described with a minimal set of parameters, their tuning is quite straightforward and done once and for all, even when the joint activity is stochastic. The tuning of the model is possible thanks to the decoupling of the plasticity process from the spine biophysics which acts as a feedforward input to the plasticity Markov chain and from the distributions of the different trajectories, which are well separated. It is expected that one can find other model versions (parameters or conceptual) instantiating our concept that also match the data well.

In our model, some CaMKII-CaN trajectories overshot the plasticity regions (e.g. Fig. 3d). Although abnormally high and prolonged calcium influx to cells can trigger cell death ${ }^{96}$, the effects of high calcium concentrations at single synapses are poorly understood. Notably, a few studies have reported evidence consistent with an overshoot, where strong synaptic calcium influx does not induce LTP $^{20,24,97}$.

Intrinsic noise is an essential component of the model. How can the synapse reliably express plasticity but be noisy at the same time ${ }^{43,44}$ ? Noise can be reduced either by redundancy or by averaging across time, also called ergodicity ${ }^{98}$. However redundancy requires manufacturing and maintaining more components, and therefore costs energy. We propose that, instead, plasticity induction is robust due to temporal averaging by slow-timescale signalling and adaptation processes. These slow variables reduce noise by averaging the faster timescale stochastic variables. This may be a reason why CaMKII uses auto-phosphorylation to sustain its activity and slow its decay time ${ }^{54,99}$. In summary, this suggests that the temporal averaging by slow variables, combined with the separability afforded by the multidimensional readout, allows synapses to tolerate noise while remaining energy-efficient.

We identified some limitations of the model. First, we modelled only a single postsynaptic spine attached to twocompartment neuron for soma and dendrite. Second, the model abstracted the complicated process of synaptic plasticity expression, and even if this replicated the "early" phase of LTP/LTD expression in the first 30-60 minutes after induction, slower protein-synthesis-dependent processes, maintenance processes, and synaptic pruning proceed at later timescales ${ }^{100}$. Third, like most biophysical models, ours contained many parameters (Methods). Although we set these to physiologically plausible values and then tuned to match the plasticity data, other combinations of parameters may fit the data equally well ${ }^{101,102}$ due to the ubiquitous phenomenon of redundancy in biochemical and neural systems ${ }^{103,104}$. Indeed synapses are quite heterogeneous in receptor and ion channel counts ${ }^{58-60,105}$, protein abundances ${ }^{106,107}$, and spine morphologies ${ }^{65,108}$, even within the subpopulation of CA1 pyramidal neuron synapses that we modelled here. It remains to be discovered how neurons tune their synaptic properties in this vast parameter space to achieve functional plasticity rules, or implement meta-plasticity ${ }^{109-111}$.

Several predictions follow from our results. Since the model respected the stochasticity of vesicle release ${ }^{112,113}$, NMDAr $^{60,74,75,114}$, and VGCC opening ${ }^{59,115,116}$, the magnitude of plasticity varied from simulation trial to trial (Methods, Fig. $3 \mathrm{~g}, 4 \mathrm{e})$. This suggests that the rules of plasticity are inherently stochastic ${ }^{63,117}$ and that the variability observed in experiments $^{9,21,24,33,34,38,40}$ is not just due to heterogeneity in 
synapse properties. By running extensive simulations over the space of protocols beyond those tested experimentally (Fig. 3h,i; $4 \mathrm{f} ; 5 \mathrm{c}, \mathrm{e}$ and $\mathrm{f} ; 6 \mathrm{e}, \mathrm{f})$, we made testable predictions for plasticity outcomes. For example, ${ }^{24}$ did not find LTD when using classic post-before-pre stimulation protocols, but the model predicted that LTD could be induced if the number of pairing repetitions was extended (Fig. h,i). The model also predicts that the lack of LTD induced by FDP in adults can be recovered using doublets or quadruplet spike protocols (Fig. 5d). We tested the model's sensitivity to spike time jitter and spike failure in the stimulation protocols (Fig. 7). Our simulations predicted that this firing variability can alter the rules of plasticity, in the sense that it is possible to add noise to cause LTP for protocols that did not otherwise induce plasticity.

What do these results imply about the rules of plasticity in vivo? First, we noticed that successful LTP or LTD induction required a balance between two types of slow variables: those that attenuate, such as presynaptic vesicle pools and dendritic $\mathrm{BaP}$, versus those that accumulate, such as slow enzymatic integration $^{62,118,119}$. This balance is reflected in the inverted-U shaped magnitude of LTP seen as a function of post-synaptic firing rate (Fig. 7h). Second, although spike timing on millisecond timescales can in certain circumstances affect the direction and magnitude of plasticity (Fig. 3), in order to drive sufficient activity of synaptic enzymes, these patterns would need to be repeated for several seconds. However, if these repetitions are subject to jitter or failures, as observed in hippocampal spike trains in vivo ${ }^{82,120}$, then the millisecond-timescale information will be destroyed as it gets averaged out across repetitions by the slow integration processes of CaMKII and CaN (Fig. 7a-d). The net implication is that millisecond-timescale structure of individual spike pairs is unlikely to play an important role in determining hippocampal synaptic plasticity in vivo ${ }^{83,88,121}$.

In summary, we presented a new type of biophysical model for plasticity induction at the rat CA3-CA1 glutamatergic synapse. Although the model itself is specific to this synapse type, the study's insights may generalise to other synapse types, enabling a deeper understanding of the rules of synaptic plasticity and brain learning.

\section{Data and Software Availability}

The code will be available on Github after peer-review process.

\section{Acknowledgments}

This work has been partially supported by the French government, through the UCAJEDI Investments in the Future project managed by the National Research Agency (ANR-15-IDEX-01), see ComputaBrain project. Funding was also provided by the UK Medical Research Council (MR/S026630/1) and from the European Union's Horizon 2020 Framework Programme for Research and Innovation under the Specific Grant Agreement No. 945539 (Human Brain Project SGA3). We thank Kim A. Blackwell, Serena M. Dudek, Matthew G. Gold, Jack R. Mellor, L. Mironov, Matthew C. Pharris, Gabriela K. Popescu and Gayle M. Wittenberg for advice and discussions.

\section{Author Contributions}

Conceptualization Y.E.R., C.O. and R.V ; Performed simulations Y,E.R; Designed the figures Y.E.R.; Original draft Y.E.R. and C.O.; Mathematical formalization Y.E.R. and R.V.; Writing, Review Editing Y.R., C.O., R.V. and H.M. ; Coding Y.E.R., C.O. and R.V.; Software R.V. ; Supervision R.V., C.O. and H.M. ; Funding H.M., C.O. ; Biological consulting C.O. and H.M. Investigation Y.E.R. and C.O. ; Project Management R.V. and H.M.

\section{Competing interests}

The authors declare no competing interests.

\section{Additional Information}

Supplementary information is available for this paper at http Correspondence and requests should be addressed to R.M. and Y.E.R.

\section{References}

1. Hebb, D. O. The organization of behavior: a neuropsychological theory (1949).

2. Blum, K. I. \& Abbott, L. F. A model of spatial map formation in the hippocampus of the rat. Neural computation $\mathbf{8}$, 85-93 (1996).

3. Gerstner, W., Kempter, R., Van Hemmen, J. L. \& Wagner, H. A neuronal learning rule for sub-millisecond temporal coding. Nature 383, 76-78 (1996).

4. Eurich, C. W., Pawelzik, K., Ernst, U., Cowan, J. D. \& Milton, J. G. Dynamics of self-organized delay adaptation. Physical Review Letters 82, 1594 (1999).

5. Debanne, D., Guerineau, N. C., Gähwiler, B. \& Thompson, S. M. Paired-pulse facilitation and depression at unitary synapses in rat hippocampus: quantal fluctuation affects subsequent release. The Journal of physiology 491, 163176 (1996).

6. Tsodyks, M. V. \& Markram, H. The neural code between neocortical pyramidal neurons depends on neurotransmitter release probability. Proceedings of the national academy of sciences $\mathbf{9 4 , 7 1 9 -}$ 723 (1997).

7. Bi, G.-q. \& Poo, M.-m. Synaptic modifications in cultured hippocampal neurons: dependence on spike timing, synaptic strength, and postsynaptic cell type. Journal of neuroscience 18, 10464-10472 (1998).

8. Markram, H., Gerstner, W. \& Sjöström, P. J. A history of spike-timing-dependent plasticity. Frontiers in synaptic neuroscience 3, 4 (2011).

9. Dudek, S. \& Bear, M. Homosynaptic long-term depression in area CA1 of hippocampus and effects of N-methyl-D-aspartate receptor blockade. Proceedings of the National Academy of Sciences $\quad 89$, 4363 (1992).

10. Sjöström, P. J., Turrigiano, G. G. \& Nelson, S. B. Rate, timing, and cooperativity jointly determine cortical synaptic plasticity. Neuron 32, 1149-1164 (2001). 
11. Artola, A., Bröcher, S \& Singer, W. Different voltagedependent thresholds for inducing long-term depression and long-term potentiation in slices of rat visual cortex. Nature 347, 69-72 (1990).

12. Magee, J. C. \& Johnston, D. A synaptically controlled, associative signal for Hebbian plasticity in hippocampal neurons. Science 275, 209-213 (1997).

13. Sjöström, P. J. \& Häusser, M. A cooperative switch determines the sign of synaptic plasticity in distal dendrites of neocortical pyramidal neurons. Neuron 51, 227-238 (2006).

14. Golding, N. L., Staff, N. P. \& Spruston, N. Dendritic spikes as a mechanism for cooperative long-term potentiation. Nature 418, 326-331 (2002).

15. Hardie, J. \& Spruston, N. Synaptic depolarization is more effective than back-propagating action potentials during induction of associative long-term potentiation in hippocampal pyramidal neurons. Journal of Neuroscience 29, 32333241 (2009).

16. Shouval, H. Z., Wang, S. S.-H. \& Wittenberg, G. M. Spike timing dependent plasticity: a consequence of more fundamental learning rules. Frontiers in computational neuroscience 4, 19 (2010).

17. Lisman, J. A mechanism for the Hebb and the antiHebb processes underlying learning and memory. Proceedings of the National Academy of Sciences $\mathbf{8 6}$, 9574-9578 (1989).

18. Shouval, H. Z., Bear, M. F. \& Cooper, L. N. A unified model of NMDA receptor-dependent bidirectional synaptic plasticity. PNAS 99, 10831-10836 (2002).

19. Karmarkar, U. R. \& Buonomano, D. V. A model of spiketiming dependent plasticity: one or two coincidence detec-

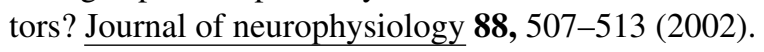

20. Yang, S.-N., Tang, Y.-G. \& Zucker, R. S. Selective induction of LTP and LTD by postsynaptic [Ca2+] i elevation. Journal of neurophysiology 81, 781-787 (1999).

21. Mizuno, T., Kanazawa, I. \& Sakurai, M. Differential induction of LTP and LTD is not determined solely by instantaneous calcium concentration: an essential involvement of a temporal factor. European Journal of Neuroscience 14, 701-708 (2001).

22. Wang, H.-X., Gerkin, R. C., Nauen, D. W. \& Bi, G.-Q. Coactivation and timing-dependent integration of synaptic potentiation and depression. Nature neuroscience $\mathbf{8}, 187-$ 193 (2005).

23. Nevian, T. \& Sakmann, B. Spine Ca2+ signaling in spiketiming-dependent plasticity. Journal of Neuroscience 26, 11001-11013 (2006).

24. Tigaret, C. M., Olivo, V., Sadowski, J. H., Ashby, M. C. \& Mellor, J. R. Coordinated activation of distinct $\mathrm{Ca} 2+$ sources and metabotropic glutamate receptors encodes Hebbian synaptic plasticity. Nature communications 7, 10289 (2016).
25. Abarbanel, H. D., Gibb, L., Huerta, R. \& Rabinovich, M. I. Biophysical model of synaptic plasticity dynamics. Biological cybernetics 89, 214-226 (2003).

26. Rubin, J. E., Gerkin, R. C., Bi, G.-Q. \& Chow, C. C. Calcium time course as a signal for spike-timing dependent plasticity. Journal of neurophysiology (2005).

27. Rackham, O., Tsaneva-Atanasova, K., Ganesh, A. \& Mellor, J. A Ca2+-based computational model for NMDA receptor-dependent synaptic plasticity at individual post-synaptic spines in the hippocampus. Frontiers in synaptic neuroscience 2, 31 (2010).

28. Clopath, C. \& Gerstner, W. Voltage and spike timing interact in STDP-a unified model. Frontiers in synaptic neuroscience 2, 25 (2010).

29. Kumar, A. \& Mehta, M. R. Frequency-dependent changes in NMDAR-dependent synaptic plasticity. Frontiers in computational neuroscience 5, 38 (2011).

30. Graupner, M. \& Brunel, N. Calcium-based plasticity model explains sensitivity of synaptic changes to spike pattern, rate, and dendritic location. Proceedings of the National Academy of Sciences 109, 3991-3996 (2012).

31. Standage, D., Trappenberg, T. \& Blohm, G. Calciumdependent calcium decay explains STDP in a dynamic model of hippocampal synapses. PloS one 9, e86248 (2014).

32. De Pittà, M. \& Brunel, N. Modulation of synaptic plasticity by glutamatergic gliotransmission: A modeling study. Neural plasticity (2016).

33. Dudek, S. M. \& Bear, M. F. Bidirectional long-term modification of synaptic effectiveness in the adult and immature hippocampus. Journal of Neuroscience 13, 29102918 (1993).

34. Meredith, R. M., Floyer-Lea, A. M. \& Paulsen, O. Maturation of long-term potentiation induction rules in rodent hippocampus: role of GABAergic inhibition. Journal of Neuroscience 23, 11142-11146 (2003).

35. Cao, G. \& Harris, K. M. Developmental regulation of the late phase of long-term potentiation (L-LTP) and metaplasticity in hippocampal area CA1 of the rat. Journal of neurophysiology 107, 902-912 (2012).

36. Cizeron, M. et al. A brain-wide atlas of synapses across the mouse lifespan. Science (2020).

37. Mulkey, R. M. \& Malenka, R. C. Mechanisms underlying induction of homosynaptic long-term depression in area CA1 of the hippocampus. Neuron 9, 967-975 (1992).

38. Inglebert, Y., Aljadeff, J., Brunel, N. \& Debanne, D. Synaptic plasticity rules with physiological calcium levels. Proceedings of the National Academy of Sciences. ISSN: 0027-8424 (2020).

39. Volgushev, M. et al. Probability of transmitter release at neocortical synapses at different temperatures. Journal of neurophysiology 92, 212-220 (2004). 
40. Wittenberg, G. M. \& Wang, S. S.-H. Malleability of spiketiming-dependent plasticity at the CA3-CA1 synapse. Journal of Neuroscience 26, 6610-6617 (2006).

41. Klyachko, V. A. \& Stevens, C. F. Temperature-dependent shift of balance among the components of short-term plasticity in hippocampal synapses. Journal of Neuroscience 26, 6945-6957 (2006).

42. Blackwell, K. T. et al. Molecular mechanisms underlying striatal synaptic plasticity: relevance to chronic alcohol consumption and seeking. European Journal of Neuroscience 49, 768-783 (2019).

43. Yuste, R., Majewska, A., Cash, S. S. \& Denk, W. Mechanisms of calcium influx into hippocampal spines: heterogeneity among spines, coincidence detection by NMDA receptors, and optical quantal analysis. Journal of Neuroscience 19, 1976-1987 (1999).

44. Ribrault, C., Sekimoto, K. \& Triller, A. From the stochasticity of molecular processes to the variability of synaptic transmission. Nature Reviews Neuroscience 12, 375 (2011).

45. Sterratt, D., Graham, B., Gillies, A. \& Willshaw, D. Principles of computational modelling in neuroscience (Cambridge University Press, 2011).

46. Buchanan, K. A. \& Mellor, J. R. The development of synaptic plasticity induction rules and the requirement for postsynaptic spikes in rat hippocampal CA1 pyramidal neurones. The Journal of Physiology 585, 429-445 (2007).

47. Golding, N. L., Kath, W. L. \& Spruston, N. Dichotomy of action-potential backpropagation in CA1 pyramidal neuron dendrites. Journal of neurophysiology $\mathbf{8 6 , 2 9 9 8 - 3 0 1 0}$ (2001).

48. Destexhe, A., Mainen, Z. F. \& Sejnowski, T. J. Kinetic models of synaptic transmission. Methods in neuronal modeling 2, 1-25 (1998).

49. Adelman, J. P., Maylie, J. \& Sah, P. Small-conductance $\mathrm{Ca} 2+$-activated $\mathrm{K}+$ channels: form and function. Annual review of physiology 74, 245-269 (2012).

50. Griffith, T., Tsaneva-Atanasova, K. \& Mellor, J. R. Control of $\mathrm{Ca} 2+$ influx and calmodulin activation by SKchannels in dendritic spines. PLoS computational biology 12 (2016).

51. Fujii, H. et al. Nonlinear decoding and asymmetric representation of neuronal input information by CaMKII $\alpha$ and calcineurin. Cell reports 3, 978-987 (2013).

52. Saraf, J. et al. A friend or foe: calcineurin across the gamut of neurological disorders. ACS central science 4, 805-819 (2018).

53. Giese, K. P., Fedorov, N. B., Filipkowski, R. K. \& Silva, A. J. Autophosphorylation at Thr286 of the $\alpha$ calciumcalmodulin kinase II in LTP and learning. Science 279, 870-873 (1998).

54. Chang, J.-Y. et al. CaMKII autophosphorylation is necessary for optimal integration of Ca2+ signals during LTP induction, but not maintenance. Neuron 94, 800-808 (2017).
55. O'Connor, D. H., Wittenberg, G. M. \& Wang, S. S.-H. Dissection of bidirectional synaptic plasticity into saturable unidirectional processes. Journal of neurophysiology $\mathbf{9 4}$, 1565-1573 (2005).

56. Otmakhov, N., Regmi, S. \& Lisman, J. E. Fast decay of CaMKII FRET sensor signal in spines after LTP induction is not due to its dephosphorylation. PLoS One 10, e0130457 (2015).

57. O'Donnell, C. \& van Rossum, M. C. Systematic analysis of the contributions of stochastic voltage gated channels to neuronal noise. Frontiers in computational neuroscience 8, 105 (2014).

58. Takumi, Y., Ramírez-León, V., Laake, P., Rinvik, E. \& Ottersen, O. P. Different modes of expression of AMPA and NMDA receptors in hippocampal synapses. Nature neuroscience 2, 618-624 (1999).

59. Sabatini, B. L. \& Svoboda, K. Analysis of calcium channels in single spines using optical fluctuation analysis. Nature 408, 589-593 (2000).

60. Nimchinsky, E. A., Yasuda, R., Oertner, T. G. \& Svoboda, K. The number of glutamate receptors opened by synaptic stimulation in single hippocampal spines. Journal of Neuroscience 24, 2054-2064 (2004).

61. Liu, G., Choi, S. \& Tsien, R. W. Variability of neurotransmitter concentration and nonsaturation of postsynaptic AMPA receptors at synapses in hippocampal cultures and slices. Neuron 22, 395-409 (1999).

62. Deperrois, N. \& Graupner, M. Short-term depression and long-term plasticity together tune sensitive range of synaptic plasticity. PLoS computational biology 16, e 1008265 (2020).

63. Bhalla, U. S. Signaling in small subcellular volumes. II. Stochastic and diffusion effects on synaptic network properties. Biophysical Journal 87, 745-753 (2004).

64. Antunes, G. \& De Schutter, E. A stochastic signaling network mediates the probabilistic induction of cerebellar long-term depression. Journal of Neuroscience 32, 92889300 (2012).

65. Bartol, T. M. et al. Computational reconstitution of spine calcium transients from individual proteins. Frontiers in synaptic neuroscience 7, 17 (2015).

66. Colbert, C. M., Magee, J. C., Hoffman, D. A. \& Johnston, D. Slow recovery from inactivation of $\mathrm{Na}+$ channels underlies the activity-dependent attenuation of dendritic action potentials in hippocampal CA1 pyramidal neurons. Journal of Neuroscience 17, 6512-6521 (1997).

67. Quintana, A. R., Wang, D., Forbes, J. E. \& Waxham, M. N. Kinetics of calmodulin binding to calcineurin. Biochemical and biophysical research communications 334, 674-680 (2005).

68. He, Y, Kulasiri, D \& Samarasinghe, S. Modelling the dynamics of CaMKII-NMDAR complex related to memory formation in synapses: The possible roles of threonine 286 autophosphorylation of CaMKII in long term potentiation. Journal of theoretical biology 365, 403-419 (2015). 
69. Wang, H. \& Wagner, J. J. Priming-induced shift in synaptic plasticity in the rat hippocampus. Journal of neurophysiology 82, 2024-2028 (1999).

70. Kealy, J. \& Commins, S. Frequency-dependent changes in synaptic plasticity and brain-derived neurotrophic factor (BDNF) expression in the CA1 to perirhinal cortex projection. Brain research 1326, 51-61 (2010).

71. Abraham, W. C., Mason-Parker, S. E., Bear, M. F., Webb, S. \& Tate, W. P. Heterosynaptic metaplasticity in the hippocampus in vivo: a BCM-like modifiable threshold for LTP. Proceedings of the National Academy of Sciences 98, 10924-10929 (2001).

72. Bienenstock, E. L., Cooper, L. N. \& Munro, P. W. Theory for the development of neuron selectivity: orientation specificity and binocular interaction in visual cortex. Journal of Neuroscience 2, 32-48 (1982).

73. Sheng, M., Cummings, J., Roldan, L. A., Jan, Y. N. \& Jan, L. Y. Changing subunit composition of heteromeric NMDA receptors during development of rat cortex. Nature 368, 144-147 (1994).

74. Popescu, G., Robert, A., Howe, J. R. \& Auerbach, A. Reaction mechanism determines NMDA receptor response to repetitive stimulation. Nature 430, 790 (2004).

75. Iacobucci, G. J. \& Popescu, G. K. NMDA receptors: linking physiological output to biophysical operation. Nature reviews Neuroscience 18, 236 (2017).

76. Rivera, C. et al. The $\mathrm{K}+/ \mathrm{Cl}-$ co-transporter $\mathrm{KCC} 2$ renders GABA hyperpolarizing during neuronal maturation. Nature 397, 251-255 (1999).

77. Rinetti-Vargas, G., Phamluong, K., Ron, D. \& Bender, K. J. Periadolescent maturation of GABAergic hyperpolarization at the axon initial segment. Cell reports 20, 21-29 (2017).

78. Isaac, J. T., Buchanan, K. A., Muller, R. U. \& Mellor, J. R. Hippocampal place cell firing patterns can induce longterm synaptic plasticity in vitro. Journal of Neuroscience 29, 6840-6850 (2009).

79. Maki, B. A. \& Popescu, G. K. Extracellular Ca2+ ions reduce NMDA receptor conductance and gating. Journal of General Physiology 144, 379-392 (2014).

80. Jahr, C. E. \& Stevens, C. F. A quantitative description of NMDA receptor-channel kinetic behavior. Journal of Neuroscience 10, 1830-1837 (1990).

81. Ngo-Anh, T. J. et al. SK channels and NMDA receptors form a $\mathrm{Ca} 2+$-mediated feedback loop in dendritic spines. Nature neuroscience 8, 642-649 (2005).

82. Fenton, A. A. \& Muller, R. U. Place cell discharge is extremely variable during individual passes of the rat through the firing field. Proceedings of the National Academy of Sciences 95, 3182-3187 (1998).

83. Graupner, M., Wallisch, P. \& Ostojic, S. Natural firing patterns imply low sensitivity of synaptic plasticity to spike timing compared with firing rate. Journal of Neuroscience 36, 11238-11258 (2016).
84. Bittner, K. C., Milstein, A. D., Grienberger, C., Romani, S. \& Magee, J. C. Behavioral time scale synaptic plasticity underlies CA1 place fields. Science 357, 1033-1036 (2017).

85. Cui, Y., Prokin, I., Mendes, A., Berry, H. \& Venance, L. Robustness of STDP to spike timing jitter. Scientific reports 8, 1-15 (2018).

86. Dobrunz, L. E., Huang, E. P. \& Stevens, C. F. Very short-term plasticity in hippocampal synapses. Proceedings of the National Academy of Sciences 94, 14843-14847 (1997).

87. Enoki, R., Hu, Y.-1., Hamilton, D. \& Fine, A. Expression of long-term plasticity at individual synapses in hippocampus is graded, bidirectional, and mainly presynaptic: optical quantal analysis. Neuron 62, 242-253 (2009).

88. Froemke, R. C. \& Dan, Y. Spike-timing-dependent synaptic modification induced by natural spike trains. Nature 416, 433 (2002).

89. Borst, J. G. G. The low synaptic release probability in vivo. Trends in neurosciences 33, 259-266 (2010).

90. Short, S. M. et al. The stochastic nature of action potential backpropagation in apical tuft dendrites. Journal of neurophysiology 118, 1394-1414 (2017).

91. Oliveira, R. F., Kim, M. \& Blackwell, K. T. Subcellular location of PKA controls striatal plasticity: stochastic simulations in spiny dendrites. PLoS Comput Biol 8, e1002383 (2012).

92. Ebner, C., Clopath, C., Jedlicka, P. \& Cuntz, H. Unifying Long-Term Plasticity Rules for Excitatory Synapses by Modeling Dendrites of Cortical Pyramidal Neurons. Cell Reports 29, 4295-4307 (2019).

93. Song, S., Miller, K. D. \& Abbott, L. F. Competitive Hebbian learning through spike-timing-dependent synaptic plasticity. Nature neuroscience 3, 919-926 (2000).

94. Pfister, J.-P. \& Gerstner, W. Triplets of spikes in a model of spike timing-dependent plasticity. Journal of Neuroscience 26, 9673-9682 (2006).

95. Badoual, M. et al. Biophysical and phenomenological models of multiple spike interactions in spike-timing dependent plasticity. International journal of neural systems 16, 79-97 (2006).

96. Zhivotovsky, B. \& Orrenius, S. Calcium and cell death mechanisms: a perspective from the cell death community. Cell calcium 50, 211-221 (2011).

97. Pousinha, P. A. et al. Physiological and pathophysiological control of synaptic GluN2B-NMDA receptors by the $\mathrm{C}$-terminal domain of amyloid precursor protein. Elife $\mathbf{6}$, e25659 (2017).

98. Sterling, P. \& Laughlin, S. Principles of neural design (MIT Press, 2015).

99. Chang, J.-Y., Nakahata, Y., Hayano, Y. \& Yasuda, R. Mechanisms of $\mathrm{Ca} 2+/$ calmodulin-dependent kinase II activation in single dendritic spines. Nature Communications 10, 2784 (2019). 
100. Bailey, C. H., Kandel, E. R. \& Harris, K. M. Structural components of synaptic plasticity and memory consolidation. Cold Spring Harbor perspectives in biology 7, a021758 (2015).

101. Marder, E. \& Taylor, A. L. Multiple models to capture the variability in biological neurons and networks. Nature neuroscience 14, 133-138 (2011).

102. Mäki-Marttunen, T., Iannella, N., Edwards, A. G., Einevoll, G. \& Blackwell, K. T. A unified computational model for cortical post-synaptic plasticity. eLife (2020).

103. Gutenkunst, R. N. et al. Universally sloppy parameter sensitivities in systems biology models. PLoS Comput Biol 3, e189 (2007).

104. Marder, E. Variability, compensation, and modulation in neurons and circuits. PNAS 108, 15542-15548 (2011).

105. Racca, C., Stephenson, F. A., Streit, P., Roberts, J. D. B. \& Somogyi, P. NMDA receptor content of synapses in stratum radiatum of the hippocampal CA1 area. Journal of Neuroscience 20, 2512-2522 (2000).

106. Shepherd, G. M. \& Harris, K. M. Three-dimensional structure and composition of $\mathrm{CA} 3 \rightarrow \mathrm{CA} 1$ axons in rat hippocampal slices: implications for presynaptic connectivity and compartmentalization. Journal of Neuroscience 18, 8300-8310 (1998).

107. Sugiyama, Y., Kawabata, I., Sobue, K. \& Okabe, S. Determination of absolute protein numbers in single synapses by a GFP-based calibration technique. Nature methods $\mathbf{2}$, 677-684 (2005).

108. Harris, K. M. \& Stevens, J. K. Dendritic spines of CA 1 pyramidal cells in the rat hippocampus: serial electron microscopy with reference to their biophysical characteristics. Journal of Neuroscience 9, 2982-2997 (1989).

109. Huang, Y.-Y., Colino, A., Selig, D. K. \& Malenka, R. C. The influence of prior synaptic activity on the induction of long-term potentiation. Science 255, 730-733 (1992).

110. Deisseroth, K, Bito, H, Schulman, H \& Tsien, R. Synaptic plasticity: a molecular mechanism for metaplasticity. Current Biology 5, 1334-1338 (1995).
111. Abraham, W. C. Metaplasticity: tuning synapses and networks for plasticity. Nature Reviews Neuroscience 9, 387387 (2008).

112. Rizzoli, S. O. \& Betz, W. J. Synaptic vesicle pools. Nature Reviews Neuroscience 6, 57-69 (2005).

113. Alabi, A. A. \& Tsien, R. W. Synaptic vesicle pools and dynamics. Cold Spring Harbor perspectives in biology 4, a013680 (2012).

114. Sinclair, D. et al. Effects of sex and DTNBP1 (dysbindin) null gene mutation on the developmental GluN2BGluN2A switch in the mouse cortex and hippocampus. Journal of neurodevelopmental disorders 8, 14 (2016).

115. Magee, J. C. \& Johnston, D. Characterization of single voltage-gated $\mathrm{Na}+$ and $\mathrm{Ca} 2+$ channels in apical dendrites of rat CA1 pyramidal neurons. The Journal of physiology 487, 67-90 (1995).

116. Iftinca, $\mathrm{M}$ et al. Temperature dependence of T-type calcium channel gating. Neuroscience 142, 1031-1042 (2006).

117. Antunes, G, Roque, A. \& Simoes-de Souza, F. Stochastic induction of long-term potentiation and long-term depression. Scientific reports 6, 30899 (2016).

118. Cai, Y., Gavornik, J. P., Cooper, L. N., Yeung, L. C. \& Shouval, H. Z. Effect of stochastic synaptic and dendritic dynamics on synaptic plasticity in visual cortex and hippocampus. Journal of neurophysiology 97, 375386 (2007).

119. Mizusaki, B. E., Li, S. S., Costa, R. P. \& Sjöström, P. J. Preand postsynaptically expressed spiking-timing-dependent plasticity contribute differentially to neuronal learning. BioRxiv, 450825 (2018).

120. Wierzynski, C. M., Lubenov, E. V., Gu, M. \& Siapas, A. G. State-dependent spike-timing relationships between hippocampal and prefrontal circuits during sleep. Neuron 61, 587-596 (2009).

121. Sadowski, J. H., Jones, M. W. \& Mellor, J. R. Sharp-wave ripples orchestrate the induction of synaptic plasticity during reactivation of place cell firing patterns in the hippocampus. Cell reports 14, 1916-1929 (2016). 


\section{Online Methods}

\section{Data and code availability}

All simulations were performed in the Julia programming language (version 1.4.2). Simulating the synapse model is equivalent to sampling a piecewise deterministic Markov process, and this relies on the Julia package PiecewiseDeterministicMarkovProcesses.jl. These simulations are event-based, and no approximation is made beyond the ones required to integrate the ordinary differential equations by the method LSODA (Livermore Solver for Ordinary Differential Equations). We run the parallel simulations in the Nef cluster operated by Inria.

\begin{tabular}{llllllll}
\hline EXPERIMENT & PAPER & REPETITIONS & FREQ $(\mathrm{Hz})$ & AGE (DAYS) & TEMP. $\left({ }^{\circ} \mathrm{C}\right)$ & $\mathrm{Ca}(\mathrm{mM})$ & $\mathrm{Mg}(\mathrm{mM})$ \\
\hline \hline STDP & 1 & 300 & 5 & 56 & 35 & 2.5 & 1.3 \\
\hline STDP & 2 & $\begin{array}{l}100, \text { positive delays } \\
150, \text { negative delays }\end{array}$ & 0.3 & $\begin{array}{l}14-20 \\
(21 \text { for LTP })\end{array}$ & $\begin{array}{l}30 \\
(30.45 \text { for LTP })\end{array}$ & $1.3-3$ & $\mathrm{Ca} / 1.5$ \\
\hline STDP & 3 & 20 & 0.2 & $9-45$ & $24-28$ & 2 & 2 \\
\hline STDP & 4 & $70-100$ & 5 & $14-21$ & $\begin{array}{l}24-30 \\
(22.5-23)\end{array}$ & 2 & 1 \\
\hline pre-burst & 1 & 300 and 900 & 3 and 5 & 56 & 35 & 2.5 & 1.3 \\
\hline FDP & 5 & 900 & $1-50$ & 35 & 35 & 2.5 & 1.5 \\
\hline FDP & 6 & 900 & 1 & $7-35$ & 35 & 2.5 & 1.5 \\
\hline TBS & 6 & $3-4(5)$ epochs & $\begin{array}{l}\text { 4Pre at } 100 \mathrm{~Hz} \\
(10 \mathrm{x} \text { at } 5 \mathrm{~Hz})\end{array}$ & 6,14 and 17 & 35 & 2.5 & 1.5 \\
\hline \multirow{2}{*}{ LFS } & 7 & $1-600$ & 1 & $12-28$ & $\begin{array}{l}30 \\
(26.5-31)\end{array}$ & 2.4 & 0 \\
\hline
\end{tabular}

Table M1. Table with the parameters extracted from the respective publications. To fit the data associated to publications displaying a parameter interval (e.g. 70 or 100) we used a value within the provided limits. Otherwise, we depict in red the value used to fit to the data. For complete data structure on these publications and the ones used for method validation see github code. We allowed the AP to be evoked by EPSPs for these protocols: ${ }^{5-7}$. Note that ${ }^{1}$ used GABA(A)r blocker modelled by turning GABAr conductance to zero. Also, ${ }^{7}$ LTD protocol used partial NMDA blocker modelled by reducing NMDA conductance by $97 \%$.

\section{Modelling procedures}

\section{Notations}

We write $\mathbf{1}_{A}$ the indicator of a set $A$ meaning that $\mathbf{1}_{A}(x)=1$ if $x$ belongs to $A$ and zero otherwise.

\section{Vesicle release and recycling}

Vesicle-filled neurotransmitters from the presynaptic terminals stimulate the postsynaptic side when successfully released. We derived a vesicle release Markov chain model based on a deterministic approach described in ${ }^{8}$ on page 183 . We denote by $\left(t_{1}, \cdots, t_{n}\right)$ the time arrivals of the presynaptic spikes.

Vesicles can be in two states, either belonging to the docked pool (with cardinal $D$ ) with fast emptying, or to the reserve pool (with cardinal $R$ ) which replenishes $\mathrm{D}^{9}$. The docked pool loses one vesicle each time a release ${ }^{10}$ occurs with transition $D \longrightarrow D-1$ (Fig. M1C). The reserve pool replenishes the docked one with transition $(R, D) \rightarrow(R-1, D+1)$. Finally, the reserve pool is replenished with rate $\left(R_{0}-R\right) / \tau_{D}^{r e f}$ with the transition $(R, D) \longrightarrow(R+1, D)$.

\begin{tabular}{lll}
\hline TRANSITION & RATE & INITIAL CONDITION \\
\hline \hline$(R, D) \rightarrow(R-1, D+1)$ & $\left(D_{0}-D\right) \cdot R / \tau_{D}$ & $D(0)=D_{0}$ \\
\hline$(R, D) \rightarrow(R+1, D-1)$ & $\left(R_{0}-R\right) \cdot D / \tau_{R}$ & $R(0)=R_{0}$ \\
\hline$(R, D) \longrightarrow(R+1, D)$ & $\left(R_{0}-R\right) / \tau_{R}^{r e f}$ & \\
\hline
\end{tabular}

Table M2. Stochastic transitions used in the pool dynamics. Note that the rates depend on the pool's cardinal ${ }^{11}$.

In addition to the stochastic dynamics in Table M2, each spike $t_{i}$ triggers a vesicle release $D \longrightarrow D-1$ with probability $p_{r e l}$ :

$$
p_{\text {rel }}\left(C a_{\text {pre }},\left[C a^{2+}\right]_{o}, D\right)=\frac{\left(C a_{\text {pre }}\right)^{s}}{\left(C a_{\text {pre }}\right)^{s}+h\left(\left[C a^{2+}\right]_{o}\right)^{s}} \mathbf{1}_{D>0}, \quad h\left(\left[C a^{2+}\right]_{o}\right)=0.654+\frac{1.349}{\left.1+e^{4 \cdot\left(\left[C a^{2+}\right]_{o}-1.708 m M\right.}\right)}
$$

which is a function of presynaptic calcium $\mathrm{Ca}$ pre and extracellular calcium concentration $\left[\mathrm{Ca}^{2+}\right]_{o}$ through the threshold $h\left(\left[\mathrm{Ca}^{2+}\right]_{o}\right)$. To decide whether a vesicle is released for a presynaptic spike $t_{i}$, we use a phenomenological model of $C a_{\text {pre }}$ (see Fig. M1A) based on 
a resource-use function ${ }^{12}$ :

$$
\begin{cases}\dot{C} a_{\text {pre }}=-\frac{C a_{\text {pre }}}{\tau_{\text {pre }}} & C a_{\text {pre }}(0)=0 \\ \dot{C} a_{\text {jump }}=\frac{1-C a_{\text {jump }}}{\tau_{\text {rec }}}-\delta_{\text {decay }} \cdot C a_{\text {jump }} \cdot C a_{\text {pre }} & C a_{\text {jump }}(0)=1 .\end{cases}
$$

Upon arrival of the presynaptic spikes, $t \in\left(t_{1}, \cdots, t_{n}\right)$, we update $C a_{\text {pre }}$ according to the deterministic jump:

$$
C a_{\text {pre }} \longrightarrow C a_{\text {pre }}+C a_{\text {jump }} \text {. }
$$

Finally, after $C a_{\text {pre }}$ has been updated, a vesicle is released with probability $p_{r e l}$ (Fig. M1B).

Parameters for the vesicle release model is given in Table M3. The experimental constraints to devise a release probability model are given by ${ }^{13}$ and $^{1}$. Because $\left[\mathrm{Ca}^{2+}\right]_{\mathrm{o}}$ modifies the release probability dynamics ${ }^{14}$, we fixed an initial release probability of $68 \%$ for $\left[\mathrm{Ca}^{2+}\right]_{\mathrm{o}}=2.5 \mathrm{mM}$ as reported by ${ }^{1}$ (initial value in Fig. M1B and D). Additionally, ${ }^{13}$ report a $38 \%$ reduction in the initial release probability when changing $\left[\mathrm{Ca}^{2+}\right]_{\mathrm{o}}$ from $2.5 \mathrm{mM}$ to $1 \mathrm{mM}$. Taking these into account, the decreasing sigmoid function in the Fig. M1E depicts our $\left[\mathrm{Ca}^{2+}\right]_{\mathrm{o}}$-dependent release probability model $\left(p_{\text {rel }}\right)$.

Fig. M1E shows that our $p_{\text {rel }}$ function is in good agreement with a previous analytical model ${ }^{14}$ suggesting that $p_{\text {rel }}\left(\left[\mathrm{Ca}^{2+}\right]_{o}\right) \propto$ $\left(\left[\mathrm{Ca}^{2+}\right]_{o}\right)^{2} \mathrm{mM}^{-2}$. Our model also qualitatively reproduces the vanishing of calcium dye fluorescence levels after $20 \mathrm{~s}$ of theta trains from $^{1}$ (in their Supplementary Materials). We interpret their fluorescence measurements as an effect of short-term depression (see Fig. M1B).

Despite our model agreeing with previous works, it is a simplified presynaptic model that does not encompass the vesicle release's highly heterogeneous nature. Vesicle release dynamics are known to be sensitivity to various experimental conditions such as temperature ${ }^{15}$, the age for some brain regions ${ }^{10}$ or magnesium concentration ${ }^{13}$. Furthermore, since our model of vesicle dynamics is simple, $\tau_{r e c}$ in Equation (1) has two roles: to delay the $p_{r e l}$ recovery caused by $C a_{p r e}$ inactivation (enforced by $\delta_{C a}$ in Equation (1)) and to prevent vesicle release after HFS induced depression ${ }^{9,14}$. Later, we incorporate a higher number of experimental parameters

\begin{tabular}{|c|c|c|}
\hline NAME & VALUE & REFERENCE \\
\hline \multicolumn{3}{|l|}{ Vesicle release model (stochastic part) } \\
\hline initial number of vesicles at $\mathrm{D}$ & $\overline{D_{0}=25}$ & 5 to $20^{9,16}$ \\
\hline initial number of vesicles at $\mathrm{R}$ & $R_{0}=30$ & 17 to 20 vesicles $^{16}$ \\
\hline $\begin{array}{l}\text { time constant } \mathrm{R} \rightarrow \mathrm{D} \\
(\mathrm{D} \text { recycling) }\end{array}$ & $\tau_{D}=5 s$ & $1 s^{9}$ \\
\hline $\begin{array}{l}\text { time constant } \mathrm{D} \rightarrow \mathrm{R} \\
(\mathrm{R} \text { mixing) }\end{array}$ & $\tau_{R}=45 s$ & $20 s$ (when depleted) to $5 \min$ (hypertonic shock) \\
\hline $\begin{array}{l}\text { time constant } 1 \rightarrow \mathrm{R} \\
(\mathrm{R} \text { recycling) }\end{array}$ & $\tau_{R}^{r e f}=40 s$ & 20 to $30 s^{9}$ \\
\hline release probability half-activation curve & $h$ & adjusted to different $\left[\mathrm{Ca}^{2+}\right]_{o}$ \\
\hline release probability sigmoid slope & $s=2$ & adjusted to different $\left[\mathrm{Ca}^{2+}\right]_{o}$ \\
\hline \multicolumn{3}{|l|}{ "Vesicle release model (deterministic part) } \\
\hline$C a_{\text {pre }}$ attenuation recovery & $\tau_{\text {pre }}=20 \mathrm{~ms}$ & $\begin{array}{r}50-500 \mathrm{~ms} \text { for with dye }{ }^{17} \\
\text { therefore }<50 \text { to } 500 \mathrm{~ms} \text { undyed (unbufered) }\end{array}$ \\
\hline deterministic jump attenuation recovery & $\tau_{r e c}=20 \mathrm{~s}$ & $\sim 20 s^{9}$ \\
\hline deterministic jump attenuation fraction & $\delta_{c a}=.0004$ & inactivation of pre calcium $^{18}$ \\
\hline
\end{tabular}
(age, temperature, $\left[\mathrm{Ca}^{2+}\right]_{\mathrm{o}},\left[\mathrm{Mg}^{2+}\right]_{\mathrm{o}}$ ) with our NMDAr model, the main postsynaptic calcium source.

Table M3. I Parameter values used in the presynaptic model. Our model does not implement a larger pool called "resting pool" containing $\sim 180$ vesicles (CA3-CA1 hippocampus) ${ }^{16}$. Terminology note: In other works, the larger pool with $\sim 180$ vesicles can be found with different nomenclatures such as "reserve pool"19 or "resting pool"16. Furthemore, the nomenclature used in our model for the reserve pool, can also be found as "recycling pool" in ${ }^{9,16}$. 
bioRxiv preprint doi: https://doi.org/10.1101/2021.03.30.437703; this version posted May 27, 2021. The copyright holder for this preprint (which was not certified by peer review) is the author/funder, who has granted bioRxiv a license to display the preprint in perpetuity. It is made available under aCC-BY-NC-ND 4.0 International license.

a

a 1 Pre, 300 at $5 \mathrm{~Hz}$

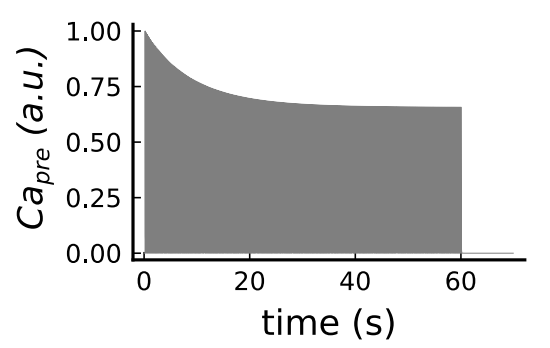

d release probability at $[\mathrm{Ca}]_{0}=2.5 \mathrm{mM}$ b $\quad p_{\text {rel }}\left(\mathrm{Ca}_{\text {pre }}\right) * \mathbb{1}$ (released)

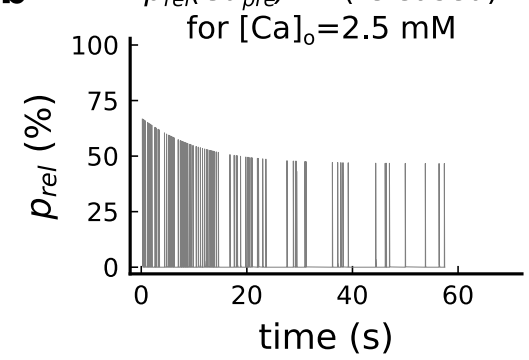

two-pool dynamics

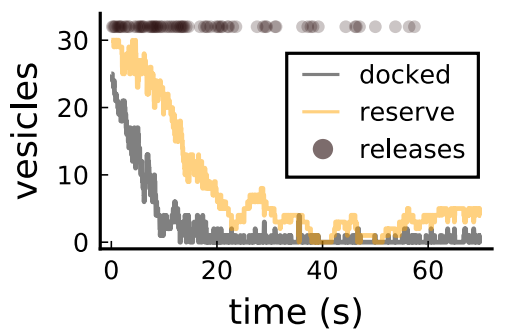

e initial release probability given extracellular calcium
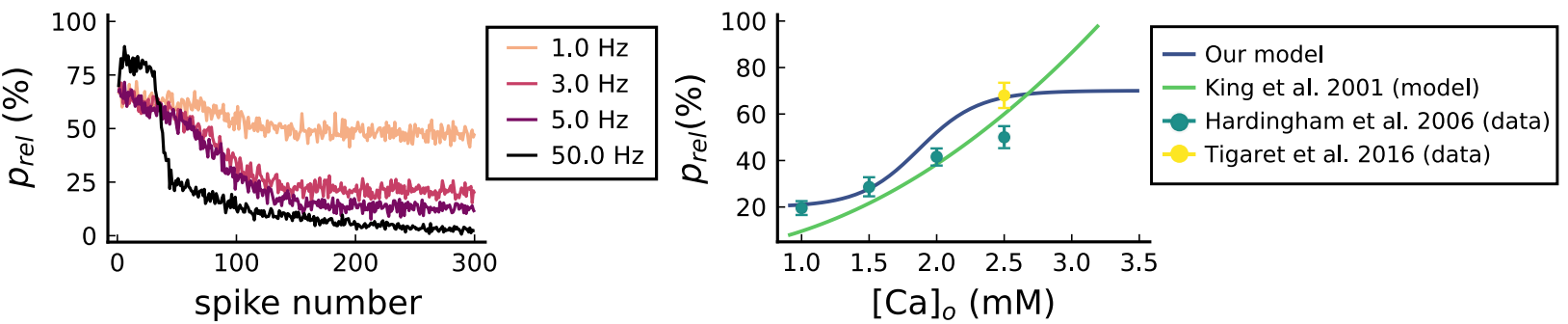

Fig. M1. I Presynaptic release. a, Presynaptic calcium in response to the protocol 1Pre, 300 at $5 \mathrm{~Hz}$ displaying adaptation. b, Release probability for the same protocol as panel A but subjected to the docked vesicles availability. c, Number of vesicles in the docked and reserve pools under depletion caused by the stimulation from panel A. d, Plot of the mean (300 samples) release probability (\%) for different frequencies for the protocol 1 Pre 300 pulses at $\left[\mathrm{Ca}^{2+}\right]_{\mathrm{o}}=2.5 \mathrm{mM}$. Note that most of the frequencies are dominated by short-term depression, and the model also displays short-term facilitation (black curve, at $50 \mathrm{~Hz}$ ). e, Release probability $(\%)$ for a single presynaptic spike given the $\left[\mathrm{Ca}^{2+}\right]_{\mathrm{o}}$. Note that ${ }^{14}$ model was multiplied by the experimentally measured release probability at $\left[\mathrm{Ca}^{2+}\right]_{0}=2 \mathrm{mM}$ since their model has this calcium concentration as the baseline. Our model also does not cover the abolishing of release probability at $\left[\mathrm{Ca}^{2+}\right]_{\mathrm{o}}=0.5 \mathrm{mM}$ which can also be difficult to experimentally measure given the rarity of events $^{13}$. 
bioRxiv preprint doi: https://doi.org/10.1101/2021.03.30.437703; this version posted May 27, 2021. The copyright holder for this preprint (which was not certified by peer review) is the author/funder, who has granted bioRxiv a license to display the preprint in perpetuity. It is made available under aCC-BY-NC-ND 4.0 International license.

\begin{tabular}{|c|c|c|}
\hline NAME & VALUE & REFERENCE \\
\hline \multicolumn{3}{|l|}{ Passive cable } \\
\hline leak reversal potential & 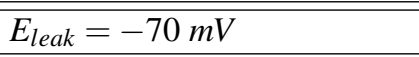 & $69 m V$ from $^{20}$ \\
\hline $\begin{array}{l}\text { membrane leak conductance } \\
\text { (for spine and passive dendrite) }\end{array}$ & $g_{\text {leak }}=4 \cdot 10^{-6} \mathrm{nS} / \mu \mathrm{m}^{2}$ & $*$ see table legend ${ }^{21}$ \\
\hline $\begin{array}{l}\text { membrane leak conductance } \\
\text { (only soma) }\end{array}$ & $g_{\text {soma }}=5.31 \cdot 10^{-3} \mathrm{nS} / \mu \mathrm{m}^{2}$ & $\begin{array}{r}3 \cdot 10^{-4} \text { to } 1.3 \cdot 10^{-3} n S / \mu m^{222} \\
47 \text { to } 2.1 \cdot 10^{3} n S \text { (NeuroElectro:CA1) }\end{array}$ \\
\hline membrane capacitance & $C_{m}=6 \cdot 10^{-3} \mathrm{pF} / \mu \mathrm{m}^{2}$ & $\begin{array}{r}1 \cdot 10^{-2} p F / \mu m^{223} \\
17 \text { to } 177 p F(\text { NeuroElectro:CA1) }\end{array}$ \\
\hline axial resistivity of cytoplasm & $R_{a}=1 \cdot 10^{-2} G \Omega \mu m$ & $2 \cdot 10^{-3} G \Omega \mu m^{24}$ \\
\hline \multicolumn{3}{|l|}{ Dendrite } \\
\hline dendrite diameter & $D_{\text {dend }}=2 \mu \mathrm{m}$ & same as ${ }^{25}$ \\
\hline dendrite length & $L_{d e n d}=1400 \mu \mathrm{m}$ & apical dendrites, 1200 to $1600 \mu m^{26}$ \\
\hline dendrite surface area & $A_{\text {dend }}=8.79 \cdot 10^{3} \mu \mathrm{m}^{2}$ & $\pi \cdot D_{\text {dend }} \cdot L_{\text {dend }}$ \\
\hline dendrite volume & $V_{\text {dend }}=4.4 \cdot 10^{3} \mu \mathrm{m}^{3}$ & $\pi \cdot\left(D_{\text {dend }} / 2\right)^{2} \cdot L_{\text {dend }}$ \\
\hline dendritic membrane capacitance & $C_{\text {dend }}=52.77 \mathrm{pF}$ & $C_{m} \cdot A_{\text {dend }}$ \\
\hline dendrite leak reversal potential & $g_{\text {leakdend }}=3.51 \cdot 10^{-2} n S$ & $g_{\text {leak }} \cdot A_{\text {dend }}$ \\
\hline dendrite axial conductance & $g_{\text {diff }}=50 n S$ & $R_{a} \cdot A_{\text {dend }}$ \\
\hline \multicolumn{3}{|l|}{ Soma } \\
\hline soma diameter & $\overline{D_{\text {soma }}=30 \mu \mathrm{m}}$ & $21 \mu m^{27}$ page 3 \\
\hline soma area (sphere) & $A_{\text {soma }}=2.82 \cdot 10^{3} \mu \mathrm{m}^{2}$ & $(4 \pi / 3) \cdot\left(D_{\text {soma }} / 2\right)^{3} ; 2.12 \cdot 10^{3} \mu m^{228}$ \\
\hline soma membrane capacitance & $C_{\text {soma }}=16.96 \mathrm{pF}$ & $C_{m} \cdot A_{\text {soma }}$ \\
\hline soma leaking conductance & $g_{\text {leaksoma }}=15 \mathrm{nS}$ & $g_{\text {soma }} \cdot A_{\text {soma }}{ }^{22}$ \\
\hline \multicolumn{3}{|l|}{ Dendritic spine } \\
\hline spine head volume & $V o_{s p}=0.03 \mu m^{3}$ & same as ${ }^{29}$ \\
\hline spine head surface & $A_{s p}=4.66 \cdot 10^{-1} \mu m^{2}$ & $4 \pi \cdot\left(3 \operatorname{Vol}_{s p} / 4 \pi\right)^{2 / 3}$ \\
\hline spine membrane capacitance & $C_{s p}=2.8 \cdot 10^{-3} p F$ & $C_{m} \cdot A_{s p}$ \\
\hline spine head leak conductance & $g_{\text {leaksp }}=1.86 \cdot 10^{-6} n S$ & $g_{\text {leak }} \cdot A_{s p}$ \\
\hline \multicolumn{3}{|l|}{ Dendritic spine neck } \\
\hline spine neck diameter & $D_{\text {neck }}=0.1 \mu \mathrm{m}$ & 0.05 to $0.6 \mu m^{30}$ \\
\hline neck length & $L_{n e c k}=0.2 \mu \mathrm{m}$ & $0.7 \pm 0.6 \mu m^{31}$ \\
\hline neck cross sectional area & $C S_{\text {neck }}=7.85 \cdot 10^{-3} \mu \mathrm{m}^{2}$ & $\pi \cdot\left(D_{\text {neck }} / 2\right)^{2}$ \\
\hline neck resistance & $g_{\text {neck }}=3.92 n S \approx 255.1 \mathrm{M} \Omega$ & $\begin{array}{r}C S_{\text {neck }} /\left(L_{\text {neck }} \cdot R_{a}\right) \\
50 \text { to } 550 M \Omega(275 \pm 27 M \Omega)^{32}\end{array}$ \\
\hline
\end{tabular}

Table M4. Parameters for the neuron electrical properties. * The membrane leak conductance in the spine is small since the spine resistance is so high that is considered infinite $\left(>10^{6} M \Omega\right)^{21}$, therefore the current mostly leaks through the neck. Additionally, the dendrite leak conductance is equally small in order to control the distance-dependent attenuation by the axial resistance term $g_{\text {BaP }}^{\text {adapt }}$ in Equations 3 and 4 . 


\section{Membrane potential and currents}

Our model is built over three compartments, a spherical dendritic spine linked by the neck to a cylindrical dendrite connected to a spherical soma. The membrane potential of these compartments satisfy the equations below (parameters in Table M4). The different currents are described in the following sections.

$$
\begin{aligned}
C_{s p} \cdot \dot{V}_{s p} & =g_{\text {neck }} \cdot\left(V_{\text {dend }}-V_{s p}\right)+g_{L}^{s p} \cdot\left(E_{\text {rev }}-V_{s p}\right)+I_{T}+I_{L}+I_{R}+I_{N M D A}+I_{A M P A}+I_{S K} \\
C_{\text {dend }} \cdot \dot{V}_{\text {dend }} & =g_{\text {Bap }}^{\text {adapt }} \cdot\left(V_{\text {soma }}-V_{\text {dend }}\right)+g_{\text {neck }} \cdot\left(V_{s p}-V_{\text {dend }}\right)+g_{L}^{\text {dend }} \cdot\left(E_{\text {rev }}-V_{\text {dend }}\right)+I_{G A B A} \\
C_{\text {soma }} \cdot \dot{V}_{\text {soma }} & =g_{\text {BaP }}^{\text {adapt }} \cdot\left(V_{\text {dend }}-V_{\text {soma }}\right)+g_{L}^{\text {soma }} \cdot\left(E_{\text {rev }}-V_{\text {soma }}\right)+\beta_{\text {age }} \cdot\left(I_{B a P}+I_{N a}\right)+I_{K}
\end{aligned}
$$

\section{Action-potential backpropagation (BaP)}

\section{Postsynaptic currents}

The postsynaptic currents are generated in the soma, backpropagated to the dendritic spine and filtered by a passive dendrite. The soma generates $\mathrm{BaPs}$ using a version of the $\mathrm{Na}+$ and $\mathrm{K}+$ channel models developed by ${ }^{33}$. The related parameters are described in Table M5 (the voltage unit is $\mathrm{mV}$ ).

\section{Sodium channel}

$$
\begin{aligned}
\alpha_{m}\left(V_{\text {soma }}\right) & =0.4 \cdot \frac{V_{\text {soma }}+30}{1-e^{-\frac{V_{\text {soma }}+30}{7.2}}} \\
\beta_{m}\left(V_{\text {soma }}\right) & =0.124 \cdot \frac{V_{\text {soma }}+30}{e^{\frac{V_{\text {soma }}+30}{7.2}}-1} \\
m_{\mathrm{inf}}\left(V_{\text {soma }}\right) & =\frac{\alpha_{m}\left(V_{\text {soma }}\right)}{\alpha_{m}\left(V_{\text {soma }}\right)+\beta_{m}\left(V_{\text {soma }}\right)} \\
m_{\tau}\left(V_{\text {soma }}\right) & =\frac{1}{\alpha_{m}\left(V_{\text {soma }}\right)+\beta_{m}\left(V_{\text {soma }}\right)} \\
\alpha_{h}\left(V_{\text {soma }}\right) & =0.01 \cdot \frac{V_{\text {soma }}+45}{e^{\frac{V_{\text {soma }}+45}{1.5}}-1} \\
\beta_{h}\left(V_{\text {soma }}\right) & =0.03 \cdot \frac{V_{\text {soma }}+45}{1-e^{-\frac{V_{\text {soma }}+45}{1.5}}} \\
\dot{h}\left(V_{\text {soma }}\right) & =\alpha_{h}\left(V_{\text {soma }}\right) \cdot(1-h)-\beta_{h}\left(V_{\text {soma }}\right) \cdot h \\
\dot{m}\left(V_{\text {soma }}\right) & =\frac{m_{\text {inf }}-m}{m_{\tau}} \\
I_{N a} & =\gamma_{N a} \cdot m^{3} \cdot h \cdot\left(\text { Erev }_{N a}-V_{\text {soma }}\right) .
\end{aligned}
$$

\section{Potassium channel}

$$
\begin{aligned}
\alpha_{n}\left(V_{\text {soma }}\right) & =e^{-0.11 \cdot\left(V_{\text {soma }}-13\right)} \\
\beta_{n}\left(V_{\text {soma }}\right) & =e^{-0.08 \cdot\left(V_{\text {soma }}-13\right)} \\
n_{\text {inf }}\left(V_{\text {soma }}\right) & =\frac{1}{1+\alpha_{n}\left(V_{\text {soma }}\right)} \\
n_{\tau}\left(V_{\text {soma }}\right) & =\max \left(50 \cdot \frac{\beta_{n}\left(V_{\text {soma }}\right)}{1+\alpha_{n}\left(V_{\text {soma }}\right)} ; 2\right) \\
\dot{n}\left(V_{\text {soma }}\right) & =\frac{n_{\text {inf }}-n}{n_{\tau}} \\
I_{K} & =\gamma_{K} \cdot n \cdot\left(\operatorname{Erev}_{K}-V_{\text {soma }}\right)
\end{aligned}
$$

To trigger a BaP, an external current $I_{B a P}$ is injected in the soma at times $t \in\left\{t_{1}, \ldots, t_{n}\right\}$ (postsynaptic input times) for a chosen duration $\delta_{i n j}$ with amplitude $I_{\text {amp }}(n A)$ :

$$
I_{B a P}=\sum_{i=1}^{n} H\left(t_{i}\right) \cdot\left(1-H\left(t_{i}+\delta_{i n j}\right)\right) \cdot I_{a m p}
$$

The current injected in the soma is filtered in a distance-dependent manner by the dendrite before it reaches the dendritic spine. The distant-dependent $\mathrm{BaP}$ amplitude attenuation changes the axial resistance $g_{B a P}^{\text {adapt }}$ (see equations 3 and 4 ) between the dendrite and the soma as follows (Fig. M2C top):

$$
g_{\text {BaP }}^{\text {adapt }}=\beta \cdot g_{\text {diff }} \cdot \beta_{\text {soma }}, \quad \quad \beta_{\text {soma }}\left(d_{\text {soma }}\right)=0.1+\frac{1.4}{1+e^{0.02 \cdot\left(d_{\text {soma }}-230.3 \mu m\right)}}
$$

where $d_{\text {soma }}$ is the distance of the spine to the soma and where the factor $\beta$ is dynamically regulated based on a resource-use equation ${ }^{12}$ with a dampening factor $\beta_{a u x}$ changing the size of the attenuation step $\delta_{\text {decay }}$ :

$$
\begin{aligned}
\dot{\beta} & =\frac{1-\beta}{\tau_{\text {rec }}}-\delta_{\text {decay }} \cdot \beta_{\text {aux }}^{-1} \cdot \beta \cdot I_{B a P}(t) \\
\dot{\beta}_{a u x} & =\frac{1-\beta_{\text {aux }}}{\tau_{\text {rec }}^{\text {aux }}}-\delta_{\text {aux }} \cdot \beta_{\text {aux }} \cdot I_{B a P}(t) .
\end{aligned}
$$


bioRxiv preprint doi: https://doi.org/10.1101/2021.03.30.437703; this version posted May 27, 2021. The copyright holder for this preprint (which was not certified by peer review) is the author/funder, who has granted bioRxiv a license to display the preprint in perpetuity. It is made available under aCC-BY-NC-ND 4.0 International license.

a distance-dependent BaP amplitude attenuation (Goldings et al. 2001)
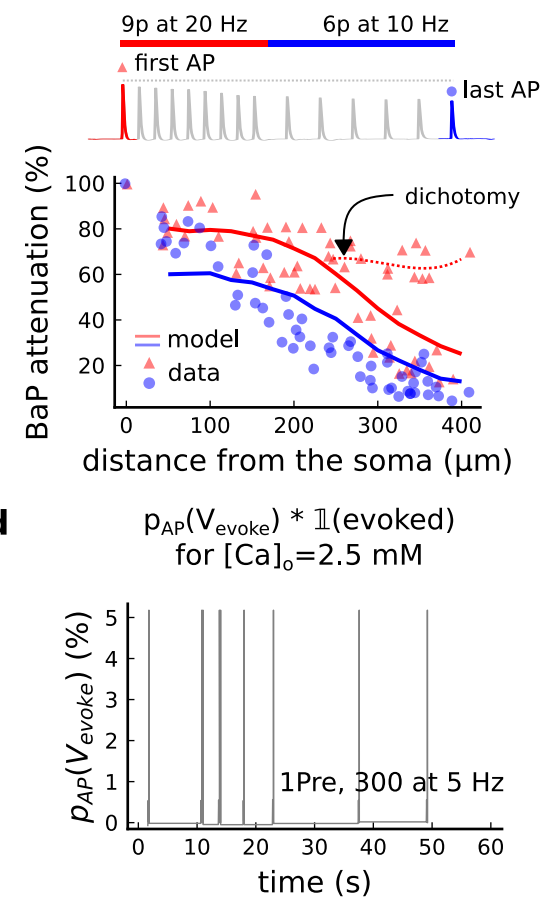

b faster AP attenuation for juvenile c rats (Buchanan \& Mellor 2007) variables implementing distance and age effects

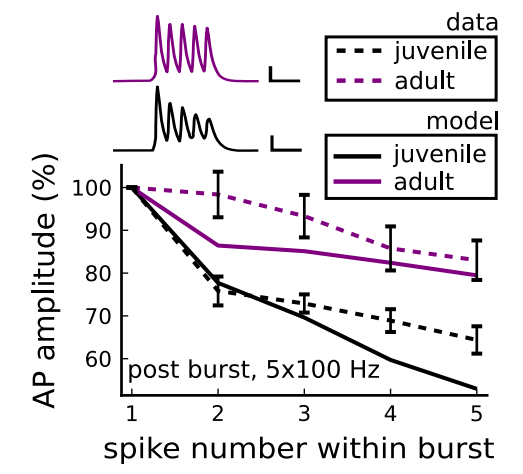

e two-pool dynamics with AP evoked by EPSP

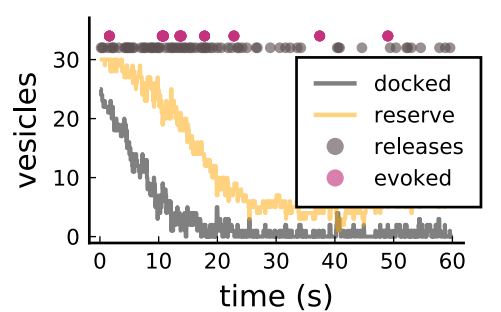

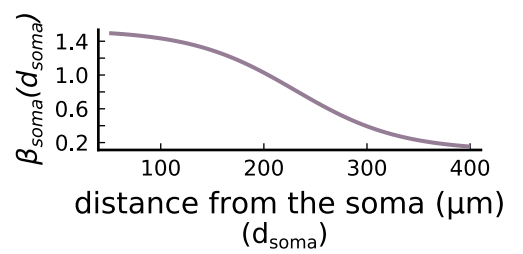

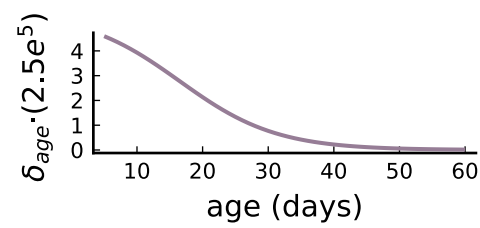

f probability of an AP being evoked by EPSP given frequency

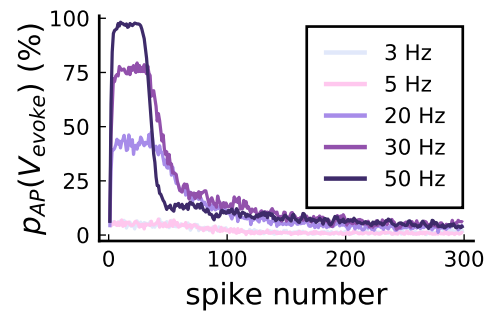

Fig. M2. I AP Evoked by EPSP. a, Model and data comparison for the distance-dependent BaP amplitude attenuation measured in the dendrite and varying the distance from the soma. The stimulation in panel A is set to reproduce the same stimulation as ${ }^{24}$. Golding measurements have neurons that are strongly attenuated and weakly attenuated (dichotomy mark represented by the dashed line). However, in this work, we consider only strongly attenuated neurons. $\mathbf{b}$, Attenuation of somatic action potential from ${ }^{37}$ and model in response to five postsynaptic spikes delivered at $100 \mathrm{~Hz}$. The value showed for the model is the spine voltage with distance from the soma set to zero (scale $25 \mathrm{~ms}, 20 \mathrm{mV}$ ).c, Top panel shows the $\beta_{\text {soma }}$ used in Equation (5) to modify the axial conductance between the soma and dendrite. Bottom panel shows the age-dependent changes in the step of the resource-use equation, in Equation (6) that accelerates the $\mathrm{BaP}$ attenuation and decreases the sodium currents in the Equation (4). d, Probability of evoking an AP multiplied by the successfully evoked AP $\left(p_{A P}\left(V_{\text {evoked }}\right) \cdot \mathbf{1}(\right.$ evoked $)$ for the protocol 1 Pre, 300 at $5 \mathrm{~Hz}(2.5 \mathrm{mM} \mathrm{Ca})$. d, Two-pool dynamics with the stimulation than panel D showing the vesicle release, the reserve and docked pools, and the evoked AP. e, Probability of evoking an AP for the protocol 1 Pre 300 pulses at different frequencies ( 3 and $5 \mathrm{~Hz}$ have the same probability).

The $\mathrm{BaP}$ attenuation model is based on ${ }^{24}$ data for strongly attenuating neurons. Therefore, the second type of attenuation (weakly attenuating) in neurons is not considered (dichotomy in Fig. M2A). Fig. M2A compares Golding data to our model and illustrates the effect of $\mathrm{BaP}$ attenuation in the upper panels of Fig. M2A and $\mathrm{B}$.

Table M5 shows the BaP attenuation parameters. The plasticity outcomes as function of the dendritic spine distance from the soma are shown in Fig. S2C and Fig. S1E.

\section{Age-dependent BaP adaptation}

Age-dependent $\mathrm{BaP}$ attenuation modifies the neuronal bursting properties through the maturation and expression of potassium and sodium channels ${ }^{34}$, therefore changing the interaction of polarizing and depolarizing currents (see Fig. M2B) ${ }^{35,36}$. We reproduce ${ }^{37}$ somatic attenuation profiles (Fig. M2B) with our model by including an age-dependent BaP amplitude attenuation factor. We define the attenuation factor $\beta_{\text {age }}$ (Fig. M2C bottom), as follows.

$$
\dot{\beta}_{\text {age }}=\frac{1-I_{\text {age }}}{\tau_{\text {rec }}^{\text {age }}}-\delta_{\text {age }} \cdot \beta_{\text {age }} \cdot I_{\text {BaP }}(t), \quad \quad \delta_{\text {rec }}^{\text {age }}=\frac{1.391 \cdot 10^{-4}}{1+e^{0.135(\text { age }-16.482 \text { days })}} .
$$

In Equation (4), the age effects are introduced by multiplying the attenuation factor $\beta_{a g e}$ by the sodium $I_{N a}$ and the external $I_{B a P}$ currents.

\section{AP evoked by EPSP}

A presynaptic stimulation triggers a BaP if sufficient depolarization is caused by the EPSPs reaching the soma ${ }^{27}$ chapter 13 . We included an option to choose whether an EPSP can evoke an AP using an event generator resembling the previous release probability model $\left(p_{\text {rel }}\right)$. Like the $p_{\text {rel }}$, the BaPs evoked by EPSPs are estimated before the postsynaptic simulation. To this, we use a variable $V_{\text {evoke }}$ which is incremented by 1 at each presynaptic time $t \in\left(t_{1}, \ldots, t_{n}\right)$ and has exponential decay:

$$
\left\{\begin{array}{l}
\dot{V}_{\text {evoke }}=-\frac{V_{\text {evoke }}}{\tau_{v}} \\
V_{\text {evoke }} \longrightarrow V_{\text {evoke }}+1
\end{array} \quad V_{\text {evoke }}(0)=0\right.
$$


bioRxiv preprint doi: https://doi.org/10.1101/2021.03.30.437703; this version posted May 27, 2021. The copyright holder for this preprint (which was not certified by peer review) is the author/funder, who has granted bioRxiv a license to display the preprint in perpetuity. It is made available under aCC-BY-NC-ND 4.0 International license.

\begin{tabular}{|c|c|c|}
\hline NAME & VALUE & REFERENCE \\
\hline \multicolumn{3}{|l|}{ Soma parameters for $\mathrm{Na}+$ and $\mathrm{K}+$ channel } \\
\hline sodium conductance & $\gamma_{N a}=8 \cdot 10^{2} n S$ & $\begin{array}{r}0.32 \mathrm{nS} / \mu \mathrm{m}^{233} \\
\text { see legend commentary }\end{array}$ \\
\hline potassium conductance & $\gamma_{K}=40 n S$ & $\begin{array}{r}0.48 \mathrm{nS} / \mu \mathrm{m}^{233} \\
\text { see legend commentary }\end{array}$ \\
\hline reversal potential sodium & Erev $_{N a}=50 \mathrm{mV}$ & 33 \\
\hline reversal potential potassium & $\operatorname{Erev}_{K}=-90 \mathrm{mV}$ & 33 \\
\hline \multicolumn{3}{|l|}{ BaP attenuation parameters } \\
\hline attenuation step factor (age) & $\delta_{\text {age }}$ & see Equation (6) and Fig. M2B and C bottom \\
\hline attenuation step factor & $\delta_{\text {decay }}=1.727 \cdot 10^{-5}$ & $\begin{array}{r}\text { adjusted to fit } \\
\qquad 24,37\end{array}$ \\
\hline auxiliary attenuation step factor & $\delta_{a u x}=2.304 \cdot 10^{-5}$ & $\begin{array}{r}\text { adjusted to fit } \\
\qquad 24,37\end{array}$ \\
\hline recovery time for the attenuation factor & $\tau_{r e c}=2 s$ & $\begin{array}{r}\text { adjusted to fit } \\
\qquad 24,37\end{array}$ \\
\hline recovery time for the auxiliary attenuation factor & $\tau_{r e c}^{a u x}=2 s$ & $\begin{array}{r}\text { adjusted to fit } \\
\qquad 24,37\end{array}$ \\
\hline recovery time for the age attenuation factor & $\tau_{\text {rec }}^{\text {age }}=0.5 s$ & $\begin{array}{r}\text { adjusted to fit } \\
24,37\end{array}$ \\
\hline \multicolumn{3}{|l|}{ AP evoked by EPSP } \\
\hline decay time for $V_{\text {evoke }}$ & $\tau_{V}=40 \mathrm{~ms}$ & 23 \\
\hline delay AP evoked by EPSP & $\delta_{\text {delay }-A P}=15 \mathrm{~ms}$ & 41 \\
\hline
\end{tabular}

Table M5. The Na+ and K+ conductances intentionally do not match the reference because models with passive dendrite need higher current input to initiate action potentials ${ }^{42}$. Therefore we set it to achieve the desired amplitude on the dendrite and the dendritic spine according to the predictions of $^{24}$ and $^{43}$.

Since the BaPs evoked by EPSP are triggered by the afferent synapses and are limited by their respective docked pools $(D)$, we use the previous $p_{r e l}$ to define the probability of an AP to occur. We test the ratio of successful releases from 25 synapses to decide if a $\mathrm{BaP}$ is evoked by an EPSP, setting a test threshold of $80 \%$. Therefore, we express the probability of evoking an AP, $p_{A P}\left(V_{e v o k e}\right)$, with the following test:

$$
\frac{\sum^{25} \mathbf{1}\left(\text { rand }<p_{\text {rel }}\left(V_{\text {evoked }},\left[C a^{2+}\right]_{o}, D\right)\right)}{25}>80 \%
$$

The EPSP summation dynamics on the soma and dendrites depend on the complex neuron morphology ${ }^{38,39}$ which was not implemented by our model. Therefore, our "AP evoked by EPSP test" intends to give a simplified way to produce BaPs similar to an integrate-and-fire model ${ }^{8}$.

Previous work suggests that BaPs can be evoked with a 5\% probability for low-frequencies ${ }^{40}$ in the Dudek and Bear 1992 experiment $\left(\left[\mathrm{Ca}^{2+}\right]_{o}=2.5 \mathrm{mM}\right)$. Our model covers this estimation, but the chance to elicit an AP increases with the frequency ${ }^{38}$. This is captured by the $V_{\text {evoke }}$ (in a integrate-and-fire fashion ${ }^{27}$ ) as shown in Fig. M2F. The Fig.s M2D and E show how a 5 Hz stimulation evokes APs. The delay between the EPSP and the evoked AP is set to $\delta_{d e l a y-A P}=15 \mathrm{~ms}$, similar to the EPSP-spike latency reported for CA1 neurons ${ }^{41}$.

\section{AMPAr}

\section{Markov chain}

The AMPAr is modeled with the Markov chain (Fig. M3) described by ${ }^{44,45}$ and adapted to temperature changes according to ${ }^{46}$. Here, we introduce the additional parameters $\rho_{f}^{A M P A}, \rho_{b}^{A M P A}$ to cover AMPAr temperature-sensitive kinetics ${ }^{46}$. The corresponding parameters are given in Table M6. 


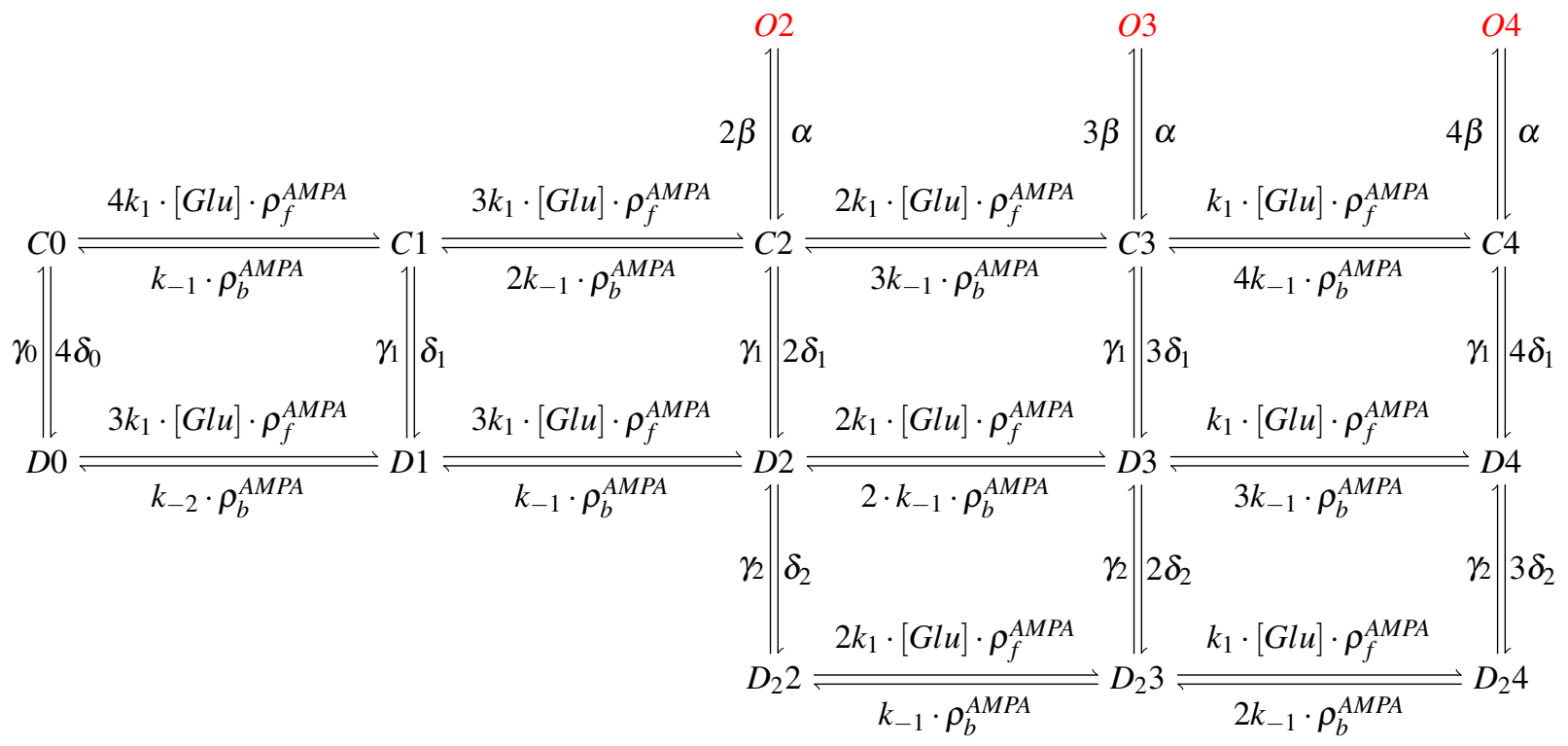

Fig. M3. AMPAr Markov chain with three sub-conductance states and two desensitisation levels. It includes parameters $\rho_{f}^{A M P A}, \rho_{b}^{A M P A}($ binding and unbinding of glutamate) which depend on temperature. Open states are $\mathrm{O} 2, \mathrm{O} 3$ and $\mathrm{O} 4$; closed states are $\mathrm{C} 0, \mathrm{C} 1, \mathrm{C} 2, \mathrm{C} 3$ and $\mathrm{C} 4$; desensitisation states are D0, D1, D2, D3 and D4; deep desensitisation states are $\mathrm{D}_{2} 2, \mathrm{D}_{2} 3$ and $\mathrm{D}_{2} 4$.

The AMPAr current is the sum of the subcurrents associated to the occupancy of the three subconductance states $\mathrm{O} 2, \mathrm{O} 3$ and $\mathrm{O} 4$ of the Markov chain in the Fig. M3 and described as follows:

$$
I_{A M P A}=\left(\operatorname{Erev}_{A M P A}-V_{s p}\right) \cdot\left(\gamma_{A 2} \cdot O 2+\gamma_{A 3} \cdot O 3+\gamma_{A 4} \cdot O 4\right) .
$$

The adaptation of the Markov chain from ${ }^{44}$ is made by changing the forward $\rho_{f}^{A M P A}$ and backward $\rho_{b}^{A M P A}$ rates in a temperaturedependent manner matching the decay time reported by ${ }^{46}$ :

$$
\rho_{f}^{A M P A}=\frac{10.273}{1+e^{-0.473 \cdot\left(T-31.724^{\circ} \mathrm{C}\right)}}, \quad \rho_{b}^{A M P A}=\frac{5.134}{1+e^{-0.367 \cdot\left(T-28.976^{\circ} \mathrm{C}\right)}} .
$$

The effects of temperature change on AMPAr dynamics are presented in Fig. M4, which also shows that the desensitisation is unaltered between temperature changes (Fig. M4B and C). The recovery time from desensitisation is the same as at room temperature ${ }^{44}$. Desensitisation measurements are required to account for a temperature-dependent change in the rates of the "vertical" transitions in Fig. M3, see ${ }^{46}$. This can be relevant for presynaptic bursts.
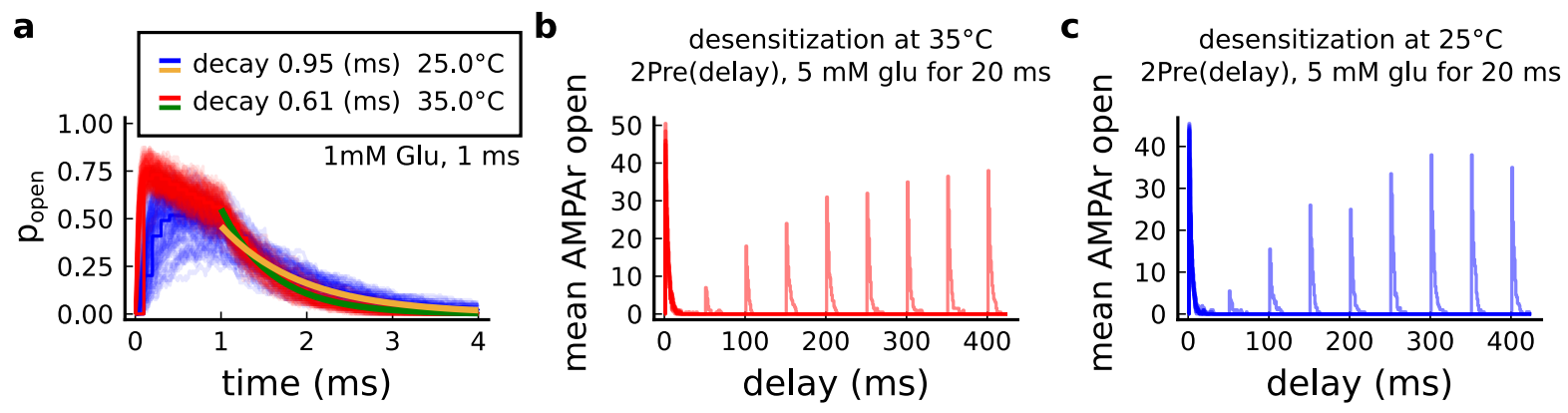

Fig. M4. I Effect of temperature in the AMPAr. a, Probability of AMPAr opening $\left(\frac{O 2+O 3+O 4}{N_{A M P A}}\right)$ and the decay time at different temperatures in response to $1 \mathrm{mM}$ glutamate during $1 \mathrm{~ms}$ (standard pulse). ${ }^{46}$ data (our model) suggests that AMPAr decay time at $35^{\circ} \mathrm{C}$ is $\sim 0.5 \mathrm{~ms}(\sim 0.6 \mathrm{~ms})$ and at $25^{\circ} \mathrm{C}$ is $\sim 0.65 \mathrm{~ms}(\sim 0.95 \mathrm{~ms})$, this shows a closer match towards more physiological temperatures. $\mathbf{b}$, Desensitisation profile of AMPAr at $35^{\circ} \mathrm{C}$ showing how many AMPAr are open in response to a glutamate saturating pulse (5 mM Glu during $20 \mathrm{~ms}$ ) separated by an interval (x-axis). c, Same as in panel $\mathrm{B}$ but for $25^{\circ} \mathrm{C}$. 
bioRxiv preprint doi: https://doi.org/10.1101/2021.03.30.437703; this version posted May 27, 2021. The copyright holder for this preprint (which was not certified by peer review) is the author/funder, who has granted bioRxiv a license to display the preprint in perpetuity. It is made available under aCC-BY-NC-ND 4.0 International license.

\begin{tabular}{|c|c|c|}
\hline NAME & VALUE & REFERENCE \\
\hline \multicolumn{3}{|l|}{ Glutamate parameters } \\
\hline duration of glutamate in the cleft & $g l u_{w i d t h}=1 \mathrm{~ms}$ & 47 \\
\hline concentration of glutamate in the cleft & $g l u_{a m p}=1 m M$ & 47 \\
\hline $\begin{array}{l}\text { glutamate variability } \\
\text { (gamma distribution } \Gamma \text { ) }\end{array}$ & $g l u_{c v}=\Gamma\left(1 / 0.5^{2}, 0.5^{2}\right)$ & 48 \\
\hline glutamate signal & Glu & $\begin{aligned} g l u_{c v} \cdot g l u_{a m p} \\
\text { for AMPAr, NMDAr and copied to GABA neurotransmitter }\end{aligned}$ \\
\hline \multicolumn{3}{|l|}{ AMPAr parameters } \\
\hline number of AMPArs & $\mathrm{N}_{A M P A}=120$ & 29 \\
\hline reversal potential & $\operatorname{Erev}_{A M P A}=0 \mathrm{mV}$ & 29 \\
\hline subconductance $\mathrm{O} 2$ & $\gamma_{A 2}=15.5 p S$ & $16.3 p S^{45}$ \\
\hline subconductance $\mathrm{O} 3$ & $\gamma_{A 3}=26 p S$ & $28.7 p S^{45}$ \\
\hline subconductance $\mathrm{O} 4$ & $\gamma_{A 4}=36.5 p S$ & $37.8 p S^{45}$ \\
\hline glu binding & $k_{1}=1.6 \cdot 10^{7} M^{-1} s^{-1}$ & 44 \\
\hline glu unbinding 1 & $k_{-1}=7400 s^{-1}$ & 44 \\
\hline glu unbinding 2 & $k_{-2}=0.41 s^{-1}$ & 44 \\
\hline closing & $\alpha=2600 s^{-1}$ & 44 \\
\hline opening & $\beta=9600 s^{-1}$ & 44 \\
\hline desensitisation 1 & $\delta_{1}=1500 s^{-1}$ & 44 \\
\hline desensitisation 2 & $\delta_{2}=170 s^{-1}$ & 44 \\
\hline desensitisation 3 & $\delta_{0}=0.003 s^{-1}$ & 44 \\
\hline re-desensitisation 1 & $\gamma_{1}=9.1 s^{-1}$ & 44 \\
\hline re-desensitisation 2 & $\gamma_{2}=42 s^{-1}$ & 44 \\
\hline re-desensitisation 3 & $\gamma_{0}=0.83 s^{-1}$ & 44 \\
\hline
\end{tabular}

Table M6. Parameter values for the AMPAr Markov chain and glutamate release affecting NMDAr, AMPAr. Properties of GABA release are the same as those for glutamate.

\section{Postsynaptic $\mathrm{Ca}^{2+}$ influx}

The effects of experimental conditions in the calcium dynamics are due to receptors, ion channels and enzymes. A leaky term models the calcium resting concentration in the Equation (8). The calcium fluxes from NMDAr and VGCCs (T, R, L types) are given in Equation (9). The diffusion term through the spine neck is expressed in Equation (10). Finally, the buffer, the optional dye and the enzymatic reactions are given in Equation (11) (parameter values given at the Table M7):

$$
\begin{aligned}
\dot{C a}= & \frac{C a_{\infty}-C a}{\tau_{C a}}+ \\
& \frac{C a_{N M D A}+I_{T}+I_{R}+I_{L}}{2 \cdot F \cdot A_{s p}}+ \\
& \frac{\max \left(C a_{\infty}, C a / 3\right)-C a}{\tau_{C a D i f f}}- \\
& B u \dot{f} f_{C a}-\text { Dye }+ \text { enzymes. }
\end{aligned}
$$

Despite the driving force to the resting concentration, $C a_{\infty}=50 \mathrm{nM}$, the tonic opening of T-type channels causes calcium to fluctuate making its mean value dependent on temperature, extracellular calcium and voltage. The effects of this tonic opening in various experimental conditions are shown in Fig. S4C. To avoid modelling dendritic calcium sources, we use a dampening term as one-third of the calcium level since calcium imaging comparing dendrite and spine fluorescence have shown this trend ${ }^{49}$. The Equation (10) implements the diffusion of calcium from the spine to the dendrite through the neck. The time constant for the diffusion coefficient $\tau_{\text {CaDiff }}$, is estimated as described $\mathrm{in}^{50}$. The calcium buffer and the optional dye are described as a two states reaction system $^{51}$ :

$$
\begin{aligned}
\text { Buff } f_{C a} & =k_{o n}^{\text {Buff }} \cdot\left(\text { Buff } f_{\text {con }}-B u f f_{C a}\right) \cdot C a-k_{o f f}^{\text {Buff }} \cdot B u f f_{C a} \\
\text { Dye } & =k_{o n}^{\text {Fluo5 }} \cdot\left(\text { Fluo5 } f_{\text {con }}-D y e\right) \cdot C a-k_{o f f}^{\text {Fluo } 5} \cdot \text { Dye. }
\end{aligned}
$$

We estimated the calcium reversal potential for the calcium fluxes using the Goldman-Hodgkin-Katz (GHK) flux equation described $\mathrm{in}^{52}$. The calcium ion permeability, $P_{C a}$, was used as a free parameter adjusting a single EPSP to produce a calcium 
amplitude of $\sim 3 \mu M$ as reported in $^{53}$.

$$
\begin{gathered}
\phi=z_{C a} \cdot V_{s p} \cdot F / R \cdot(T+273.15 K) \\
\Phi_{C a}=-P_{C a} \cdot z_{C a} \cdot F \cdot \phi \cdot \frac{[\mathrm{Ca}]_{i}-[\mathrm{Ca}]_{o} \cdot e^{-\phi}}{1-e^{-\phi}}
\end{gathered}
$$

\begin{tabular}{|c|c|c|}
\hline NAME & VALUE & REFERENCE \\
\hline \multicolumn{3}{|l|}{ Buffer and dye } \\
\hline association buffer constant & $k_{\text {on }}^{\text {Buff }}=0.247 \mu M^{-1} \mathrm{~ms}^{-1}$ & 29 \\
\hline dissociation buffer constant & $k_{o f f}^{\text {Buff }}=0.524 \mathrm{~ms}^{-1}$ & 29 \\
\hline buffer concentration & Buff $f_{\text {con }}=62 \mu M$ & $76.7 \mu M^{29}$ \\
\hline \multicolumn{3}{|l|}{ Calcium dynamics } \\
\hline Calcium baseline concentration & $C a_{\infty}=50 n M$ & $37 \pm 5$ to $54 \pm 5 n M^{17}$ \\
\hline Calcium decay time & $\tau_{C a}=10 \mathrm{~ms}$ & $\begin{array}{l}50 \text { to } 500 \mathrm{~ms} \text { for with dye }{ }^{17} \\
\text { therefore }<50 \text { to } 500 \mathrm{~ms} \text { undyed (unbufered) }\end{array}$ \\
\hline Calcium diffusion & $D_{C a}=0.3338 \mu m^{2} m s^{-1}$ & 0.22 to $0.4 \mu m^{2} m^{-129,50}$ \\
\hline Calcium diffusion time constant & $\tau_{\text {CaDiff }}=\frac{V o l_{s p}}{2 D_{C a}^{2} \cdot D_{\text {neck }}}+\frac{L_{\text {neck }}^{2}}{2 D_{C a}}=0.5 \mathrm{~ms}$ & $8 m s$ for a $V_{s p}=0.7 \mu m^{350}$ \\
\hline \multicolumn{3}{|l|}{ GHK } \\
\hline temperature & $T=35^{\circ} \mathrm{C}$ & converted to Kelvin in the Equation (12) given the protocol \\
\hline faraday constant & $F=96.485 \mathrm{C} \mathrm{mol}^{-1}$ & 52 \\
\hline gas constant & $R=8.314 J^{-1} \mathrm{~mol}^{-1}$ & 52 \\
\hline Calcium permeability & $P_{C a}=0.045 \mu m m^{-1}$ & $\begin{array}{r}\text { adjusted to produce } 3 \mu M \text { Calcium in response to a Glu release } \\
\text { supplementary files from }\end{array}$ \\
\hline Calcium ion valence & $z_{C a}=2$ & 52 \\
\hline
\end{tabular}

$\Phi_{C a}$ is used to determine the calcium influx through NMDAr and VGCC in the Equations 13, 14, 15 and 16.

Table M7. Postsynaptic calcium dynamics parameters.

\section{NMDAr - GluN2A and GluN2B Markov chain}

In the hippocampus, the NMDAr are principally heteromers composed of the obligatory subunit GluN1 and either the GluN2A or GluN2B subunits. These N2 subunits guide the activation kinetics of these receptors with the GluN1/GLUN2B heteromers displaying slow kinetics $(\sim 250 \mathrm{~ms})$ and the GluN1/GluN2A heteromers displaying faster kinetics ( $\sim 50 \mathrm{~ms})$. We modeled both NMDA subtypes. The NMDAr containing GluN2A is modeled with the following Markov chain ${ }^{54}$ where we introduce the additional parameters $\rho_{f}^{N M D A}, \rho_{b}^{N M D A}$ :

$$
A_{0} \underset{k_{-a} \cdot \rho_{b}^{N M D A}}{\stackrel{k_{a} \cdot[G l u] \cdot \rho_{f}^{N M D A}}{\rightleftharpoons}} A_{1} \underset{k_{-b} \cdot \rho_{b}^{N M D A}}{\stackrel{k_{b} \cdot[G l u] \cdot \rho_{f}^{N M D A}}{\rightleftharpoons}} A_{2} \underset{k_{-c} \cdot \rho_{b}^{N M D A}}{\stackrel{k_{c} \cdot \rho_{f}^{N M D A}}{\rightleftharpoons}} A_{3} \frac{k_{d} \cdot \rho_{f}^{N M D A}}{\underset{k_{-d} \cdot \rho_{b}^{N M D A}}{\rightleftharpoons}} A_{4} \underset{k_{-e} \cdot \rho_{b}^{N M D A}}{\stackrel{k_{e} \cdot \rho_{f}^{N M D A}}{\rightleftharpoons}} A_{O 1} \frac{k_{f} \cdot \rho_{f}^{N M D A}}{\rightleftharpoons k_{-f} \cdot \rho_{b}^{N M D A}} A_{O 2}
$$

The NMDAr containing GluN2B is modeled with a Markov chain based on the above GluN2A scheme. We decreased the rates by $\sim 75 \%$ in order to match the GluN2B decay at $25^{\circ} \mathrm{C}$ as published in ${ }^{55}$.

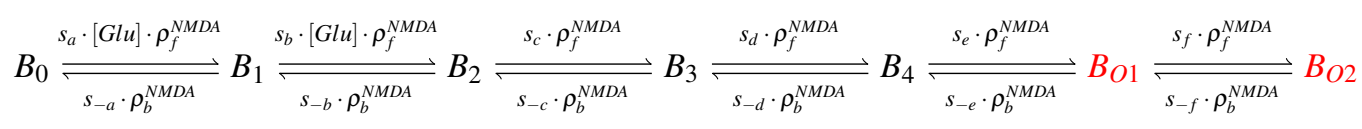

The different rates are given in Table M8.

\section{NMDAr and age switch}

The age-dependent expression ratio of the subtypes GluN2A and GluN2B $\left(r_{\text {age }}\right)$ was obtained from hippocampal mice data ${ }^{56}$. We added noise to this ratio causing $\sim 1$ NMDAr subunit to flip towards GluN2A or GluN2B (see Fig. M5E). The population of 15 NMDAr is divided in the two subtypes according to the ratio plotted in Fig. M5B as function of age. The ratio to define the number 
bioRxiv preprint doi: https://doi.org/10.1101/2021.03.30.437703; this version posted May 27, 2021. The copyright holder for this preprint (which was not certified by peer review) is the author/funder, who has granted bioRxiv a license to display the preprint in perpetuity. It is made available under aCC-BY-NC-ND 4.0 International license.
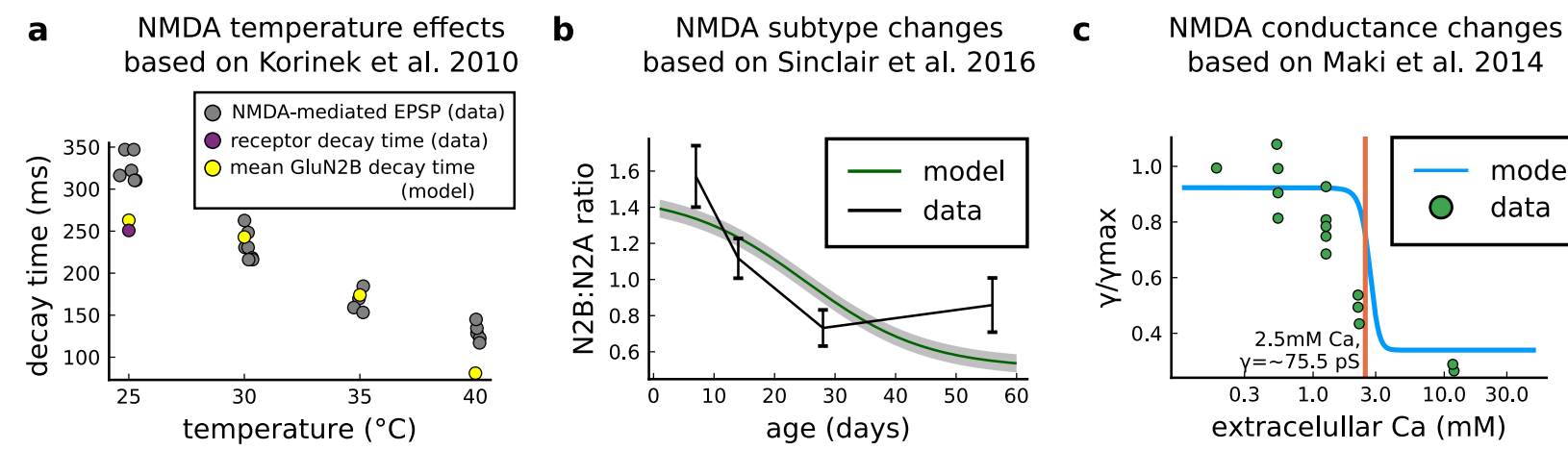

\section{d Temperature factor for NMDA}
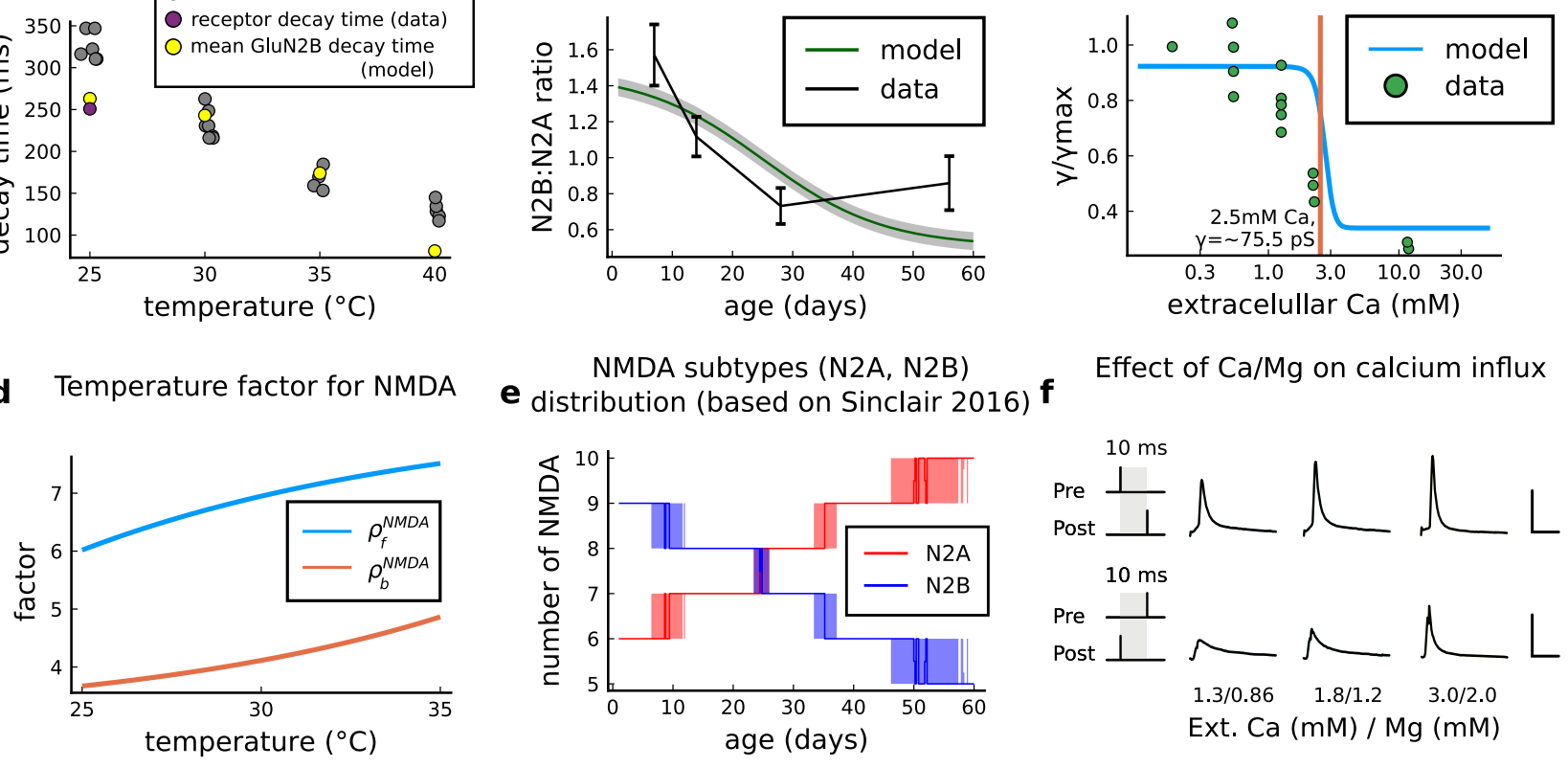

Fig. M5. I NMDAr changes caused by age, temperature and extracellular and magnesium concentrations in the aCSF.A Decay time of the NMDAr-mediated EPSP recorded from neocortical layer II/III pyramidal neurons (grey) ${ }^{57}$ compared to the decay time from the GluN2B channel estimated by our model (yellow) and data from Iacobussi's single receptor recording (purple) ${ }^{55}$. b. Comparison of our implementation of GluN2B:GluN2A ratio and the GluN2B:GluN2A ratio from the mice's CA1. c, Comparison of our implementation of NMDAr conductance change in response to the extracellular against data ${ }^{58}$. d, Forward and backwards temperature factors implemented to approximate NMDAr subtypes decay times at room temperature ${ }^{55}$ and temperature changes observed in ${ }^{57}$. e, NMDAr subtypes number on our model given age. We add noise to have a smoother transition between different ages. f, Calcium concentration changes for causal and anticausal protocols in response to different aCSF calcium and magnesium compositions with fixed $\mathrm{Ca} / \mathrm{Mg}$ ratio (1.5). Scale $50 \mathrm{~ms}$ and $5 \mu M$.

NMDAr subtypes as function of age reads:

$$
\begin{aligned}
r_{\text {age }} & =0.507+\frac{0.964}{1+e^{0.099 \cdot(\text { age }-25.102 \text { days })}}+\mathscr{N}(0,0.05) \\
N_{G l u N 2 B} & =\text { round }\left(\frac{N_{N M D A} \cdot r_{\text {age }}}{r_{\text {age }}+1}\right) \\
N_{G l u N 2 A} & =\text { round }\left(\frac{N_{N M D A}}{r_{\text {age }}+1}\right) .
\end{aligned}
$$

The round term in the two previous equations ensures that we have an integer value for the NMDAr subtypes, making the stair shaped curve seen in Fig. M5E.

\section{NMDAr and temperature}

We adjusted the GluN2A and GluN2B forward and backward rates to follow the temperature effects on NMDAr-mediated EPSP ${ }^{57}$ (see Fig. M5A and D). Because GluN2B dominates the NMDAr-mediated EPSP, we fit its decay time on the NMDAr-mediated EPSP as function of temperature as reported by ${ }^{57}$ using a logistic functions $\rho_{f}^{N M D A}$ and $\rho_{b}^{N M D A}$. The decay time comparison is shown in Fig. M5A. Then, we applied the same temperature factor $\rho_{f}^{N M D A}$ and $\rho_{b}^{N M D A}$ for GluN2A. The decay times of GluN2A and GluN2B are similar to the ones reported by Iacobucci ${ }^{55}$. The forward and backward factors are described as follows:

$$
\rho_{f}^{N M D A}=-1230.680+\frac{1239.067}{1+e^{-0.099 \cdot\left(T+37.631^{\circ} C\right)}}, \quad \rho_{b}^{N M D A}=3.036+\frac{1621.616}{1+e^{-0.106 \cdot\left(T-98.999^{\circ} C\right)}} .
$$




\section{NMDAr current and $\mathrm{Ca}^{2+}$-dependent conductance}

NMDAr conductance is modulated by external calcium and is modelled according to the next equations using NMDAr subconductances $A_{O 1}$ and $A_{O 2}(\mathrm{GluN} 2 \mathrm{~A})$, and $B_{O 1}$ and $B_{O 2}(\mathrm{GluN} 2 \mathrm{~B})$.

$$
\begin{aligned}
\gamma_{N M D A} & =33.949+\frac{58.388}{1+e^{4 \cdot\left(\left[C a^{2+}\right]_{o}-2.701 m M\right)}} p S \\
B\left(V_{s p},[M g]_{o}\right) & =\frac{1}{1+\frac{[M g]_{o}}{3.57} \cdot e^{-0.062 \cdot V_{s p}}} \\
N M D A & =\left(B_{O 1}+B_{O 2}+A_{O 1}+A_{O 2}\right) \cdot B\left(V_{s p},[M g]_{o}\right) \cdot \gamma_{N M D A} \\
I_{N M D A} & =\left(\text { Erev }_{N M D A}-V_{s p}\right) \cdot N M D A
\end{aligned}
$$

We now modify the conductance function $\gamma_{N M D A}$ reported by ${ }^{58}$. The reported NMDAr conductance at $\left[\mathrm{Ca}^{2+}\right]_{o}=1.8 \mathrm{mM}$ is $53 \pm 5 p S$. Here, we used the higher conductance $91.3 \mathrm{pS}$ for NMDAr (for both subtypes) at $\left[\mathrm{Ca}^{2+}\right]_{o}=1.8 \mathrm{mM}$ to compensate for the small number of NMDArs reported by ${ }^{59}$. Hence, we cover ${ }^{58}$ data differently to account for this constraint: this caused a right-shift in the NMDA-conductance curve (Fig. M5C). The calcium influx $C a_{N M D A}$ is modulated by the GHK factor, Equation (12), as function of the internal and external calcium concentrations and the spine voltage:

$$
C a_{N M D A}=f_{C a} \cdot \Phi_{C a} \cdot N M D A .
$$

The combined effect of Magnesium ${ }^{60}$ and extracellular Calcium concentration are displayed in Fig. M5F.

\section{$\operatorname{GABA}(\mathrm{A})$ receptor \\ Markov chain}

We used the GABA(A) receptor Markov chain (Fig. M6) presented in Bush and Sakmann 199063,64 and we estimated temperature adaptations using Otis and Mody $1992^{65}$ measurements.

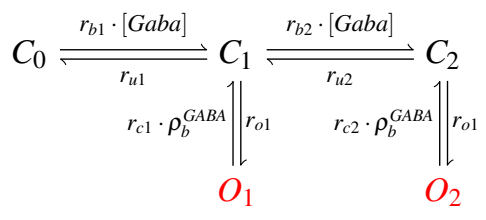

Fig. M6. I GABAr Markov chain model. Closed states $\left(C_{0}, C_{1}\right.$ and $\left.C_{2}\right)$ open in response to GABAr and can go either close again or open $\left(O_{1}\right.$ and $\left.\mathrm{O}_{2}\right)$

\section{$\operatorname{GABA}(A) r$ and temperature}

Because the amplitude of GABA(A) current is altered by the GABAr shift ${ }^{66}$ during development, we applied temperature changes only to the closing rates using a logistic the function $\rho_{b}^{G A B A}$ estimated by fitting ${ }^{65}$ measurements (data comparison in the Fig. M7B and E).

$$
\rho_{b}^{G A B A}=1.470-\frac{-1.279}{1+e^{0.191 \cdot(T-32.167)}} .
$$

\section{GABA(A)r current and age switch}

The GABA(A)r-driven current changes during development ${ }^{3}$ passing from depolarizing (excitatory) to hyperpolarizing (inhibitory) ${ }^{67}$. That is, the reversal potential of chloride ions permeating $\operatorname{GABA}(\mathrm{A}) \mathrm{r}$ shifts from above the membrane resting potential (inward driving force - excitatory) to below the membrane resting potential (outward driving force - inhibitory) ${ }^{66}$. Such effect mediated by chloride ions is associated with the $\mathrm{KCC} 2$ pump ( $\mathrm{K} \mathrm{Cl}$ co-transporter) which becomes efficient in extruding chloride ions during maturation ${ }^{66}$. To cover the GABA(A)r shift, we fit the chloride reversal potential $\left(E_{r e v}^{C l}\right)$ using the data published by ${ }^{66}$ (Fig. M7C):

$$
\begin{gathered}
E_{\text {rev }}^{C l}=-92.649+\frac{243.515}{1+e^{0.091 \cdot(\text { age }-0.691 \text { days })}} \\
I_{G A B A}=\left(O_{1}+O_{2}\right) \cdot\left(E_{\text {rev }}^{C l}-V_{\text {dend }}\right) \cdot \gamma_{G A B A} .
\end{gathered}
$$

Table M9 presents the parameters to model the GABAr. 
bioRxiv preprint doi: https://doi.org/10.1101/2021.03.30.437703; this version posted May 27, 2021. The copyright holder for this preprint (which was not certified by peer review) is the author/funder, who has granted bioRxiv a license to display the preprint in perpetuity. It is made available under aCC-BY-NC-ND 4.0 International license.

\begin{tabular}{|c|c|c|}
\hline NAME & VALUE & ENCE \\
\hline \multicolumn{3}{|l|}{ NMDAr (GluN2A) } \\
\hline glutamate binding & $k_{a}=34 \mu M^{-1} s^{-1}$ & 54 \\
\hline glutamate binding & $k_{b}=17 \mu M^{-1} s^{-1}$ & 54 \\
\hline forward rate & $k_{c}=127 s^{-1}$ & 54 \\
\hline forward rate & $k_{d}=580 s^{-1}$ & 54 \\
\hline opening rate & $k_{e}=2508 s^{-1}$ & 54 \\
\hline opening rate & $k_{f}=3449 s^{-1}$ & 54 \\
\hline closing rate & $k_{-f}=662 s^{-1}$ & 54 \\
\hline closing rate & $k_{-e}=2167 s^{-1}$ & 54 \\
\hline backward rate & $k_{-d}=2610 s^{-1}$ & 54 \\
\hline backward rate & $k_{-c}=161 s^{-1}$ & 54 \\
\hline glutamate unbinding & $k_{-b}=120 s^{-1}$ & 54 \\
\hline glutamate unbinding & $k_{-a}=60 s^{-1}$ & 54 \\
\hline \multicolumn{3}{|l|}{ NMDAr (GluN2B) } \\
\hline glutamate binding & $s_{b}=0.25 k_{b}$ & adapted from GluN2 $\mathrm{A}^{54,55}$ \\
\hline glutamate binding & $s_{c}=0.25 k_{c}$ & adapted from GluN2 $\mathrm{A}^{54,55}$ \\
\hline forward rate & $s_{c}=0.25 k_{c}$ & adapted from GluN2 $\mathrm{A}^{54,55}$ \\
\hline forward rate & $s_{d}=0.25 k_{d}$ & adapted from GluN2A ${ }^{54,55}$ \\
\hline opening rate & $s_{e}=0.25 k_{e}$ & adapted from GluN2 $\mathrm{A}^{54,55}$ \\
\hline opening rate & $s_{f}=0.25 k_{f}$ & adapted from GluN2 $\mathrm{A}^{54,55}$ \\
\hline closing rate & $s_{-f}=0.23 k_{-f}$ & adapted from GluN2A $\mathrm{A}^{54,55}$ \\
\hline closing rate & $s_{-e}=0.23 k_{-e}$ & adapted from GluN2A $\mathrm{A}^{54,55}$ \\
\hline backward rate & $s_{-d}=0.23 k_{-d}$ & adapted from GluN2A ${ }^{54,55}$ \\
\hline backward rate & $s_{-c}=0.23 k_{-c}$ & adapted from GluN2 $\mathrm{A}^{54,55}$ \\
\hline glutamate unbinding & $s_{-b}=0.23 k_{-b}$ & adapted from GluN2A ${ }^{54,55}$ \\
\hline glutamate unbinding & $s_{-a}=0.23 k_{-a}$ & adapted from GluN2A $\mathrm{A}^{54,55}$ \\
\hline \multicolumn{3}{|l|}{ other parameters } \\
\hline total number of NMDAr & $N_{N M D A}=15$ & $5-30^{29,47,59}$ \\
\hline distribution of GluN2A and GluN2B & defined by $r_{\text {age }}$ & 56 \\
\hline NMDAr conductance depending on calcium & $\gamma_{N M D A}$ & 58 \\
\hline NMDAr reversal potential & Erev $_{N M D A}=0 m V$ & 61 \\
\hline fraction of calcium carried by NMDAr & $f_{C a}=0.1$ & 62 \\
\hline
\end{tabular}

Table M8. NMDAr parameters.

\begin{tabular}{llr}
\hline NAME & VALUE & ENCE \\
\hline \hline GABA(A) receptor & & \\
\hline \hline number of GABA & $N_{G A B A}=34$ & $30^{68}$ \\
\hline chloride reversal potential & see age-dependent equation & \\
\hline GABAr conductance & $\gamma_{G A B A}=36 p S$ & $27 S^{69}$ \\
\hline binding & $r_{b 1}=20 \cdot 10^{6} M^{-1} s^{-1}$ & same as ${ }^{63}$ \\
\hline unbinding & $r_{u 1}=4.6 \cdot 10^{3} s^{-1}$ & same as \\
\hline binding & $r_{b 2}=10 \cdot 10^{6} M^{-1} s^{-1}$ & same as \\
\hline unbinding & $r_{u 2}=9.2 \cdot 10^{3} s^{-1}$ & same as \\
\hline opening pore & $r_{r o 1}=3.3 \cdot 10^{3} s^{-1}$ & same as \\
\hline opening pore & $r_{r o 2}=10.6 \cdot 10^{3} s^{-1}$ & same as \\
\hline closing pore & $r_{c 2}=400 s^{-1}$ & based on ${ }^{63,65}$ \\
\hline closing pore & $r_{c 2}=9.8 \cdot 10^{3} s^{-1}$ & based on ${ }^{63,65}$ \\
\hline
\end{tabular}

Table M9. GABAr parameters. 
bioRxiv preprint doi: https://doi.org/10.1101/2021.03.30.437703; this version posted May 27, 2021. The copyright holder for this preprint (which was not certified by peer review) is the author/funder, who has granted bioRxiv a license to display the preprint in perpetuity. It is made available under aCC-BY-NC-ND 4.0 International license.
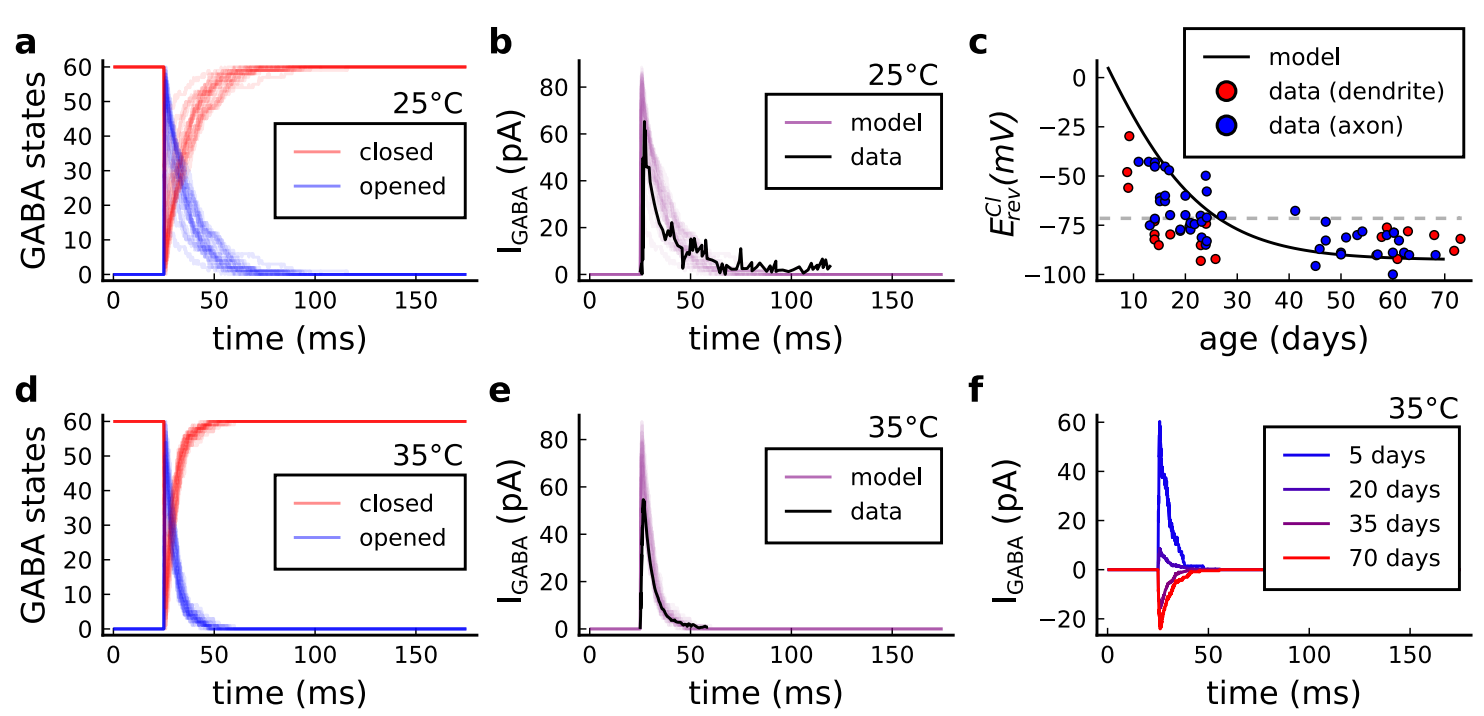

Fig. M7. I GABA(A)r current, kinetics and chloride reversal potential. a, States of GABA(A)r Markov chain at $25^{\circ} \mathrm{C}$ in response to a presynaptic stimulation. Opened $=O_{1}+O_{2}$, closed $=C_{0}+C_{1}+C_{2}$. b. Model and data comparison ${ }^{65}$ for GABA(A)r current at $25^{\circ} \mathrm{C}$. Even though data were recorded from $\mathrm{P} 70$ at $25^{\circ} \mathrm{C}$ and $\mathrm{P} 15$ at $35^{\circ} \mathrm{C}$, we normalize the amplitude to invert the polarity and compare the decay time. This is done since the noise around P15 can either make GABAr excitatory or inhibitory as shown by $E_{c l}$ data in panel C. c, Chloride reversal potential $\left(E_{r e v}^{C l}\right)$ fitted to ${ }^{66}$ data. Note we used both profiles from axon and dendrite age-depended $E_{\text {rev }}^{C l}$ changes since exclusive dendrite data is scarce. d, States of simulated from GABA(A)r Markov chain at $35^{\circ} \mathrm{C}$ in response to a presynaptic stimulation. e, Model and data comparison ${ }^{65}$ for $\mathrm{GABA}(\mathrm{A}) \mathrm{r}$ current at $25^{\circ} \mathrm{C}$ (same normalization as in panel B). f, Change in the polarization of GABA(A)r currents given the age driven by the $E_{r e v}^{C l}$.

\section{VGCC - T, R and L type \\ Markov chain}

A stochastic VGCC model was devised using the channel gating measurements from CA1 rat's (2-8 weeks) pyramidal neurons by Magee and Johnston 1995 at room temperature ${ }^{70}$. Our model has three different VGCC subtypes described by the Markov chains in Fig. M8: the T-type (low-voltage), the R-type (medium-to-high-voltage) and the L-type (high-voltage).

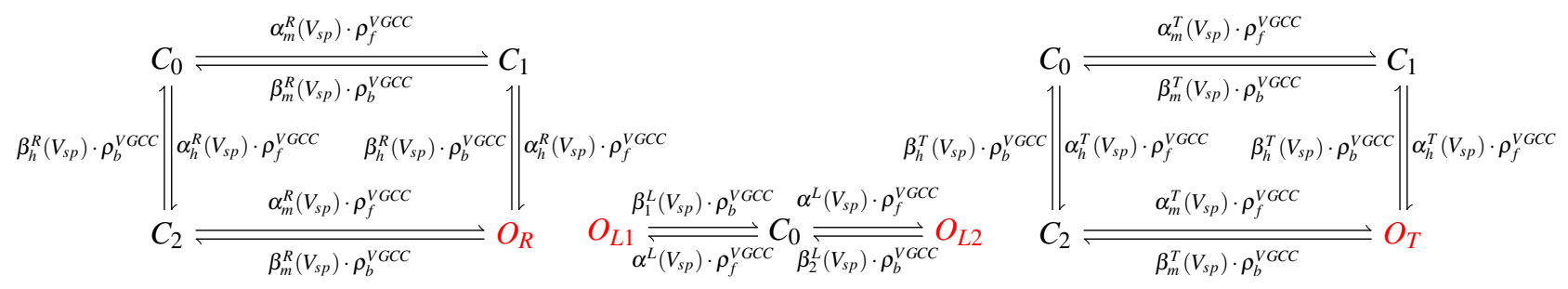

Fig. M8. From left to right, R-, L-, and T-type VGCCs Markov chain adapted from Magee and Johnston 1995 $5^{70}$. The R- (left scheme) and T- type (right scheme) have a single open state (red colour), respectively, $O_{r}$ and $O_{T}$. The L-type VGCC (middle) has two open states, $O_{L 1}$ and $O_{L 2}$.

The VGCC Markov chain derived from Magee and Johnston $1995^{70}$ are composed of two gates (h,m) for T- (Fig. M9A and D) and R-types (Fig. M9B and E) and a single gate for L-type (Fig. M9C), as described in the equations below. 
R-type h-gate rates

$$
\begin{aligned}
\tau_{h}^{R \star} & =100 \\
h_{\text {inf }}^{R \star}\left(V_{s p}\right) & =\frac{1}{1+e^{\frac{V_{s p}+39}{9.2}}} \\
\alpha_{h}^{R}\left(V_{s p}\right) & =\frac{h_{\text {inf }}^{R}}{\tau_{h}^{R}} \\
\beta_{h}^{R}\left(V_{s p}\right) & =\frac{1-h_{\text {inf }}^{R}}{\tau_{h}^{R}}
\end{aligned}
$$

R-type m-gate rates

$$
\begin{aligned}
\beta_{m}^{R \star} & =40 \\
m_{\text {inf }}^{R \star} & =\frac{1}{1+e^{\frac{3-10}{8}}} \\
\alpha_{m}^{R \star} r & =\beta_{m}^{R \star} \cdot \frac{m_{\text {inf }}^{R \star}}{1-m_{\text {inf }}^{R \star}} \\
\tau_{m}^{R} & =\frac{1}{\alpha_{m}^{R \star}+\beta_{m}^{R \star}} \\
m_{\text {inf }}^{R} & =\frac{1}{1+e^{\frac{3-V_{s p}}{8}}} \\
\alpha_{m}^{R}\left(V_{s p}\right) & =\frac{m_{\text {inf }}^{R}}{\tau_{m}^{R}} \\
\beta_{m}^{R}\left(V_{s p}\right) & =\frac{1-m_{\text {inf }}^{R}}{\tau_{m}^{R}}
\end{aligned}
$$

T-type h-gate rates

$$
\begin{aligned}
\tau_{h}^{T \star} & =50 \\
h_{\text {inf }}^{T \star}\left(V_{s p}\right) & =\frac{1}{1+e^{\frac{V_{s p}+70}{6.5}}} \\
\alpha_{h}^{T}\left(V_{s p}\right) & =\frac{h_{\text {inf }}^{T}}{\tau_{h}^{T}} \\
\beta_{h}^{T}\left(V_{s p}\right) & =\frac{1-h_{\text {inf }}^{T}}{\tau_{h}^{T}}
\end{aligned}
$$

T-type m-gate rates

$$
\begin{aligned}
\beta_{m}^{T \star} & =1 \\
m_{i n f}^{T \star} & =\frac{1}{1+e^{\frac{-32+20}{7}}} \\
\alpha_{m}^{T \star} r & =\beta_{m}^{T \star} \cdot \frac{m_{i n f}^{T *}}{1-m_{\text {inf }}^{T \star}} \\
\tau_{m}^{T} & =\frac{1}{\alpha_{m}^{T \star}+\beta_{m}^{T \star}} \\
m_{i n f}^{T} & =\frac{1}{1+e^{\frac{-32-V_{s p}}{7}}} \\
\alpha_{m}^{T}\left(V_{s p}\right) & =\frac{m_{i n f}^{T}}{\tau_{m}^{T}} \\
\beta_{m}^{T}\left(V_{s p}\right) & =\frac{1-m_{i n f}^{T}}{\tau_{m}^{T}}
\end{aligned}
$$

L-type rates

$$
\begin{aligned}
& \alpha^{L}\left(V_{s p}\right)=\frac{0.83}{1+e^{\frac{13.7-V_{s p}}{6.1}}} \\
& \beta_{1}^{L}\left(V_{s p}\right)=\frac{0.53}{1+e^{\frac{V_{s p}-11.5}{6.4}}} \\
& \beta_{2}^{L}\left(V_{s p}\right)=\frac{1.86}{1+e^{\frac{V_{s p}-18.8}{6.17}}}
\end{aligned}
$$

VGCC and temperature

We used the same temperature factor for every VGCC subtype, respectively $\rho_{f}^{V G C C}$ and $\rho_{b}^{V G C C}$ (see Fig. M9F), as follows:

$$
\begin{aligned}
& \rho_{f}^{V G C C}=2.503-\frac{0.304}{1+e^{1.048 \cdot(T-30.668)}} \\
& \rho_{b}^{V G C C}=0.729+\frac{3.225}{1+e^{-0.330 \cdot(T-36.279)}} .
\end{aligned}
$$

The VGCC subtypes are differently sensitive to temperature, with temperature factors for decay times ranging from $2^{71}$ to 50 -fold ${ }^{72}$. It further complicates if T-type isoforms are considered. Indeed, they can have temperature factors that accelerate or slow down the kinetics. For instance, when passing from room to physiological temperatures, the isoform $\mathrm{Ca}_{v} 3.3$ has a closing time $\sim 50 \%$ faster ${ }^{71}$ and the isoform $\mathrm{Ca}_{v} 3.1$ becomes $\sim 15 \%$ slower. To simplify, the same temperature factor is adopted to all VGCC subtypes.

\section{VGCC currents}

The VGCC currents are integrated to the dendritic spine and estimated using the GHK Equation (12), as follows:

$$
\begin{aligned}
& I_{T}=\gamma_{T} \cdot \Phi_{C a} \cdot O_{T} \\
& I_{R}=\gamma_{R} \cdot \Phi_{C a} \cdot O_{R} \\
& I_{L}=\gamma_{L} \cdot \Phi_{C a} \cdot\left(O_{L 1}+O_{L 2}\right)
\end{aligned}
$$


bioRxiv preprint doi: https://doi.org/10.1101/2021.03.30.437703; this version posted May 27, 2021. The copyright holder for this preprint (which was not certified by peer review) is the author/funder, who has granted bioRxiv a license to display the preprint in perpetuity. It is made available under aCC-BY-NC-ND 4.0 International license.

Table M10 presents the parameters to model the VGCC channels.

\begin{tabular}{llc}
\hline NAME & VALUE & ENCE \\
\hline \hline VGCC & & \\
\hline \hline VGCC T-type conductance & $\gamma_{C a T}=12 p S$ & same as ${ }^{70}$ \\
\hline VGCC R-type conductance & $\gamma_{C a R}=17 p S$ & same as \\
\hline VGCC L-type conductance & $\gamma_{C a L}=27 p S$ & same as ${ }^{70}$ \\
\hline number of VGCCs & 3 for each subtype & 1 to $20^{73}$ \\
\hline
\end{tabular}

Table M10. VGCC parameters

a

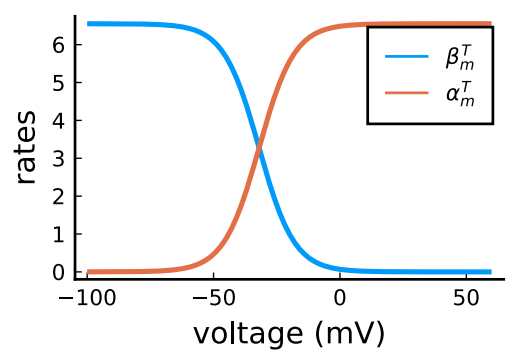

d T-type, low-voltage h-gate

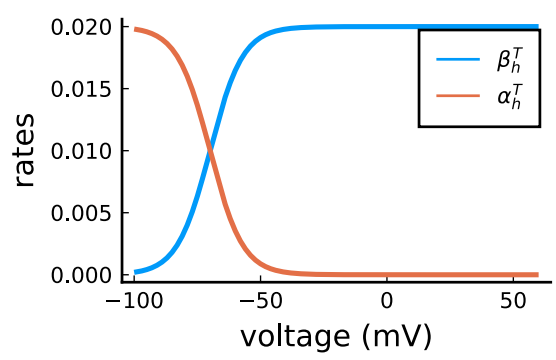

b

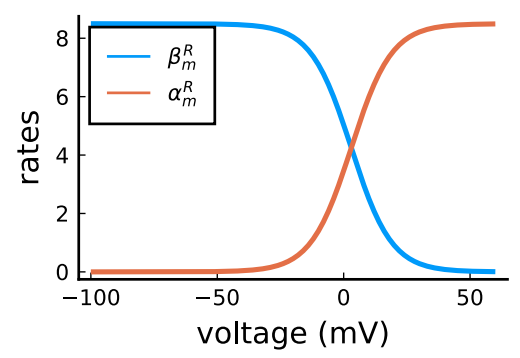

e R-type, high-voltage h-gate

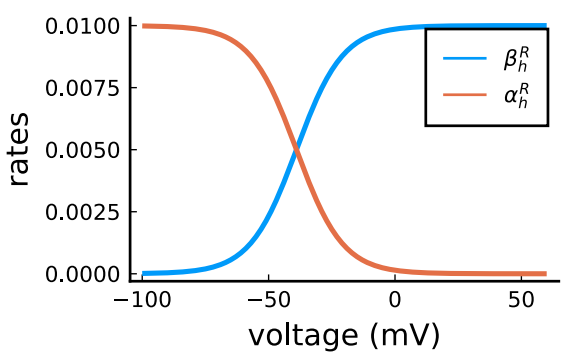

C L-type, high-voltage m-gate

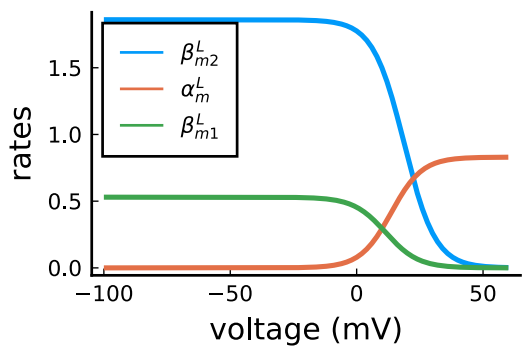

$\mathbf{f}$

VGCC temperature factor

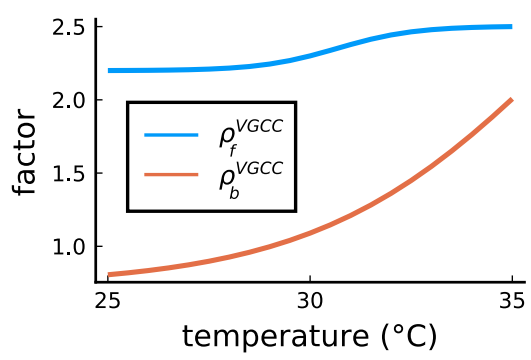

Fig. M9. I VGCC rates and temperature factors. a, Activation $\left(\alpha_{m}\left(V_{s p}\right)\right)$ and deactivation rates $\left(\beta_{m}\left(V_{s p}\right)\right)$ for the T-type m-gate. b, Activation $\left(\alpha_{m}\left(V_{s p}\right)\right)$ and deactivation rates $\left(\beta_{m}\right)$ for the R-type m-gate. c, Activation $\left(\alpha_{m}\left(V_{s p}\right)\right)$ and both deactivation rates $\left(\beta_{2}^{L}\left(V_{s p}\right)\right.$ and $\left.\beta_{2}^{1}\left(V_{s p}\right)\right)$ for the L-type VGCC. d, Activation $\left(\alpha_{h}\left(V_{s p}\right)\right)$ and deactivation rates $\left(\beta_{h}\left(V_{s p}\right)\right)$ for the T-type h-gate. e, Activation $\left(\alpha_{h}\left(V_{s p}\right)\right)$ and deactivation rates $\left(\beta_{h}\left(V_{s p}\right)\right)$ for the R-type h-gate. f, Temperature factor applied to all the rates, forward change $\left(\rho_{f}^{V G C C}\right)$ for the $\alpha$ rates and backward change $\left(\rho_{b}^{V G C C}\right)$ for the $\beta$ rates.

\section{SK channel}

The small potassium (SK) channel produces hyperpolarizing currents which are enhanced in the presence of intracellular calcium elevations. The SK channel current was based on the description of ${ }^{62}$ as follows:

$$
\begin{aligned}
r(C a) & =\frac{C a^{\sigma}}{C a^{\sigma}+h_{S K}^{\sigma}} \\
m_{s k} & =\frac{r(C a) \cdot \rho_{f}^{S K}-m_{s}}{\tau_{S K} \cdot \rho_{b}^{S K}} \\
I_{S K} & =\gamma_{S K} \cdot\left(E_{r e v}^{S K}-V_{s p}\right) \cdot m_{s k} \cdot N_{S K} .
\end{aligned}
$$

We chose a temperature factor to decrease the decay time of hyperpolarizing currents by a factor of two when passing from physiological to room temperature. Despite that the ences for temperature effects on the SK channel are few, a report ${ }^{74}$ suggests a left-ward shift in the SK half-activation when changing from $37^{\circ} \mathrm{C}\left(h_{S K}=0.38 \pm 0.02 \mu M\right)$ to $25^{\circ} \mathrm{C}\left(h_{S K}=0.23 \pm 0.01 \mu M\right)$; that is a $65 \%$ decrease.

$$
\rho_{b}^{S K}=149.37-\frac{147.61}{1+e^{0.093 \cdot(T-98.85 C)}}, \quad \quad \rho_{f}^{S K}=0.005+\frac{2.205}{1+e^{-0.334 \cdot(T+25.59 C)}}
$$

Table M11 presents the parameters to model the SK channel. 
bioRxiv preprint doi: https://doi.org/10.1101/2021.03.30.437703; this version posted May 27, 2021. The copyright holder for this preprint (which was not certified by peer review) is the author/funder, who has granted bioRxiv a license to display the preprint in perpetuity. It is made available under aCC-BY-NC-ND 4.0 International license.

\begin{tabular}{llr}
\hline NAME & VALUE & ENCE \\
\hline \hline SK channel & & \\
\hline \hline number of SK channels & $N_{S K}=15$ & $10-200^{75}$ \\
\hline SK conductance & $\gamma_{S K}=10 \mathrm{pS}$ & ${ }^{76}$ \\
\hline SK reversal potential & $E_{\text {rev }}^{S K}=-90 \mathrm{mV}$ & ${ }^{62}$ \\
\hline SK half-activation & $h_{S K}=0.333 \mu \mathrm{M}$ & ${ }^{62}$ \\
\hline SK half-activation slope & $\sigma=6$ & 4 in $^{62}$ \\
\hline SK time constant & $\tau_{S K}=6.3 \mathrm{~ms}$ & ${ }^{62}$ \\
\hline
\end{tabular}

Table M11. SK channel parameters.

\section{Enzymes - CaM, CaN and CaMKII Markov chain}

To model the enzymes dynamics, we adapted a monomeric CaM-CaMKII Markov chain from ${ }^{77}$ which builds over ${ }^{78}$. Our adaptation incorporates a simplified $\mathrm{CaN}$ reaction which only binds to fully saturated $\mathrm{CaM}$. That is, $\mathrm{CaM}$ bound to four calcium ions on $\mathrm{N}$ and $\mathrm{C}$ terminals (see Markov chain in the Fig. M10). A consequence of the Pepke coarse-grained model is that calcium binds and unbinds simultaneously from the CaM terminals $(\mathrm{N}, \mathrm{C})$. We assumed no dephosphorylation reaction between CaMKII and CaN since ${ }^{79}$ experimentally suggested that no known phosphatase affects CaMKII decay time which is probably caused only by CaM untrapping ${ }^{79}$. This was previously theorized by ${ }^{80}$ 's model, and it is reflected in Chang data ${ }^{53,77}$. The structure of the corresponding Markov chain is shown in Fig. M10.

Chang's data ${ }^{77}$ provides a high-temporal resolution fluorescence measurements for CaMKII in the spines of rat's CA1 pyramidal neurons and advances the description of CaMKII self-phosphorylation (at room temperature). We modified Chang's model of CaMKII unbinding rates $k_{2}, k_{3}, k_{4}, k_{5}$ to fit CaMKII dynamics at room/physiological temperature as shown by ${ }^{53}$ supplemental files. Previous modelling of CaMKII ${ }^{77,78}$ used a stereotyped waveform with no adaptation to model calcium. Our contribution to CaMKII modelling was to use calcium dynamics sensitive to the experimental conditions to reproduce CaMKII data, therefore, allowing us to capture physiological temperature measurements from ${ }^{53}$. Note that CaMKII dynamics has two time scales and we only capture the fastest one (after stimulation ceases, $60 \mathrm{~s}$ ) and the relative amplitude of CaMKII between the different temperatures. The slowest one occurs at the end of the stimulus, close to the maximum (Fig. M11A); this can be caused by the transient volume increase in the spine as measured by ${ }^{53}$.

Table M12 shows the concentration of the enzymes and Table M13 shows the parameters to model enzymes reactions in the Fig. M10.

\begin{tabular}{llr}
\hline NAME & VALUE & REFERENCE \\
\hline \hline Enzyme concentrations & & \\
\hline \hline free CaM concentration & $C_{a} M_{c o n}=30 \mu \mathrm{M}$ & 81 \\
\hline free $\mathrm{KCaM}$ concentration & $m K C a M_{\text {con }}=70 \mu \mathrm{M}$ & ${ }^{2,, 83}$ \\
\hline free CaN concentration & $m C a N_{c o n}=20 \mu \mathrm{M}$ & $5-20 \mu \mathrm{M}^{84}$ \\
\hline
\end{tabular}

Table M12. Concentration of each enzyme.

\section{Temperature effects on enzymatic-activity}

We then included temperature factors in the coarse-grained model using Chang data ${ }^{77}$, as shown in Fig. M11. For CaMKII, we fitted the modified dissociation rates of the phosphorylation states $k_{2}, k_{3}$ and $k_{5}$ to match the data on relative amplitude and decay time using the following logistic function:

$$
\rho_{b}^{C a M K I I}=162.171-\frac{161.426}{1+e^{0.511\left(T-45.475^{\circ} \mathrm{C}\right)}} .
$$

For CaN, we fit the ${ }^{85}$, data at $25^{\circ} \mathrm{C}$ as seen in Fig. M12A. However, since CaN-CaM dissociation rates at physiological temperatures were not found, we set the temperature factor to $\mathrm{CaN}$ that fits the outcomes of the protocols we proposed to reproduce. A reference value from the CaN-AKAP79 complex $^{86}$ showed a $Q_{10}=4.46=\left(2.19 s^{-1} / 9.78 s^{-1}\right)$ which is nearly the temperature factor used in our model for $\mathrm{CaM}$. Therefore, both the association and dissociation rates are modified using the following logistic functions:

$$
\begin{aligned}
& \rho_{f}^{C a N}=2.503-\frac{0.304}{1+e^{1.048\left(T-30.668^{\circ} \mathrm{C}\right)}} \\
& \rho_{b}^{C a N}=0.729+\frac{3.225}{1+e^{-0.330\left(T-36.279^{\circ} \mathrm{C}\right)}} .
\end{aligned}
$$


bioRxiv preprint doi: https://doi.org/10.1101/2021.03.30.437703; this version posted May 27, 2021. The copyright holder for this preprint (which

was not certified by peer review) is the author/funder, who has granted bioRxiv a license to display the preprint in perpetuity. It is made available under aCC-BY-NC-ND 4.0 International license.

CaM-Ca and CaM-CaN reactions

KCaM-Ca reactions

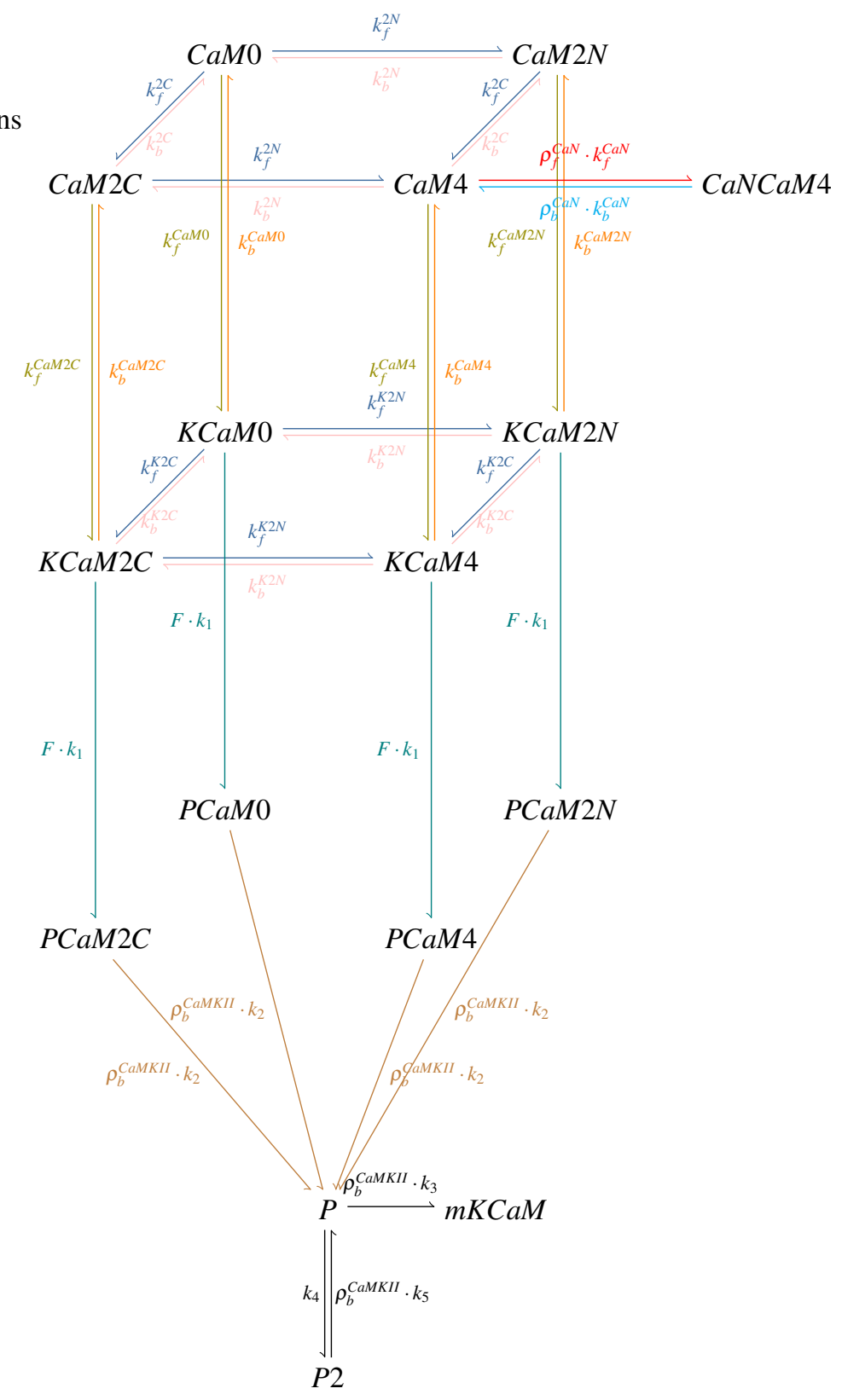

Fig. M10. I Coarse-grained model of CaM, CaMKII and CaN adapted from ${ }^{77}$ and $^{78}$ The reaction description matches with the color: Releases $2 \mathrm{Ca}$, consumes 2Ca, consumes mKCaM, releases mKCaM, releases CaM2C, CaM2N, CaM0, CaM2N, releases mCaN, consumes mCaN, phoshorylate $\mathrm{K}$ units to $\mathrm{P}$ units, phosphorylated states and dephosphorylation. 
bioRxiv preprint doi: https://doi.org/10.1101/2021.03.30.437703; this version posted May 27, 2021. The copyright holder for this preprint (which was not certified by peer review) is the author/funder, who has granted bioRxiv a license to display the preprint in perpetuity. It is made available under aCC-BY-NC-ND 4.0 International license.

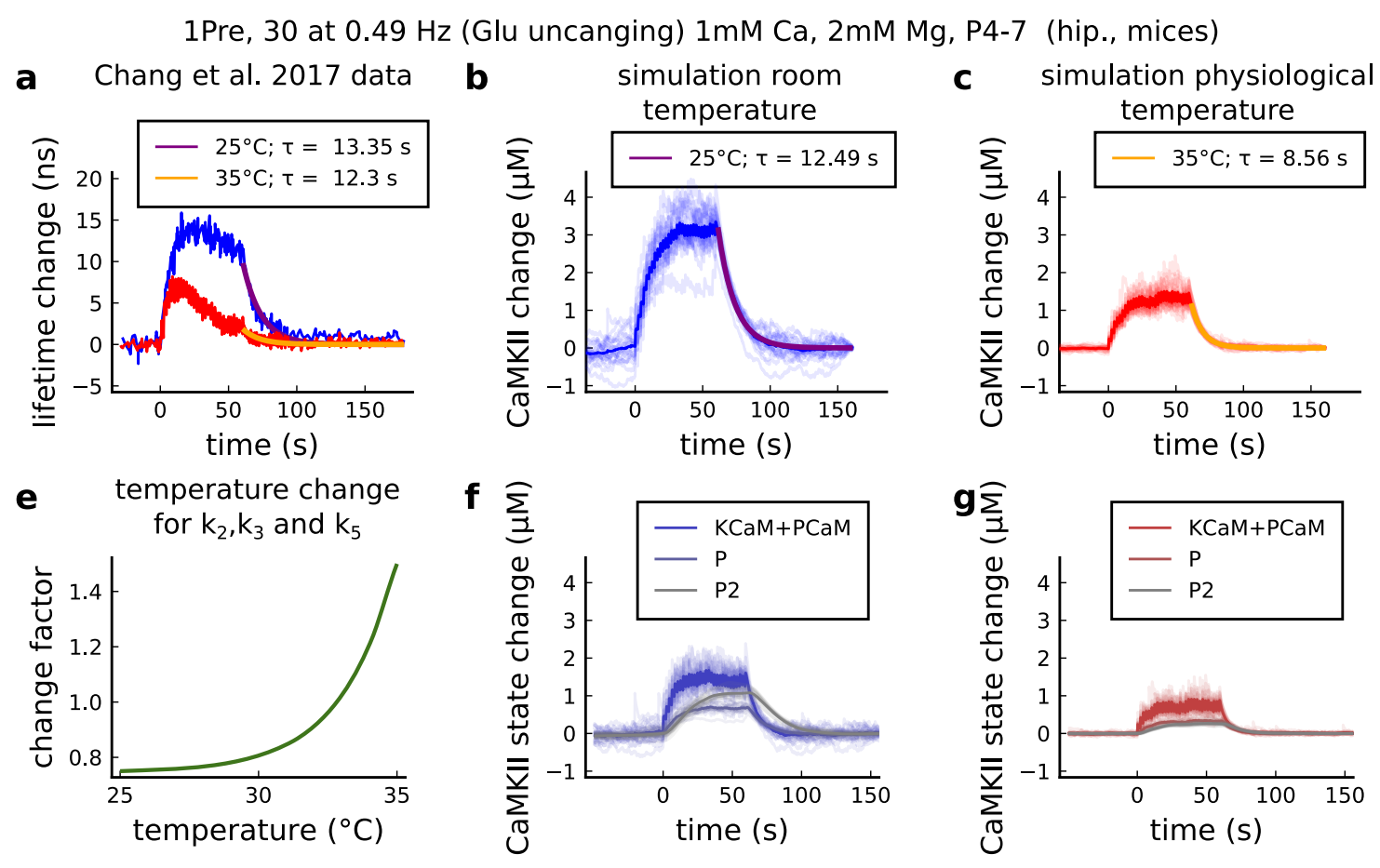

Fig. M11. I CaMKII temperature changes in our model caused by 1 Pre, 30 at $0.49 \mathrm{~Hz}$ with Glutamate uncaging (no failures allowed), $1 \mathrm{Mm}$ Ca, 2mM Mg, P4-7 organotypic slices from mice hippocampus. a, CaMKII fluorescent probe lifetime change measured by ${ }^{53}$ for $25^{\circ}($ blue $)$ and $35^{\circ} \mathrm{C}(\mathrm{red})^{53}$. The decay time $(\tau)$ was estimated by fitting the decay after the stimulation ( 30 pulses at $0.49 \mathrm{~Hz}$ ) using a single exponential decay, $y=a \cdot e^{-t \cdot b} ; \tau=1 \backslash b$. b. Simulation of the CaMKII concentration change (with respect to the baseline) at $25^{\circ}$ in response to same protocol applied in the panel A. The simulations on the panels B, C, E and F show the mean of 20 samples. c, Same as in panel B but for $35^{\circ} \mathrm{C}$. d, Estimated temperature change factor for the dissociation rates $k_{2}, k_{3}$ and $k_{5}$ in the Markov chain at the Fig. M10. e, Change in the concentration of the CaMKII states $\left(25^{\circ} \mathrm{C}\right)$ which are summed to compose CaMKII change in the panel B. f, Same as in panel $\mathrm{E}$ for $35^{\circ} \mathrm{C}$ with reference to the panel $\mathrm{C}$.

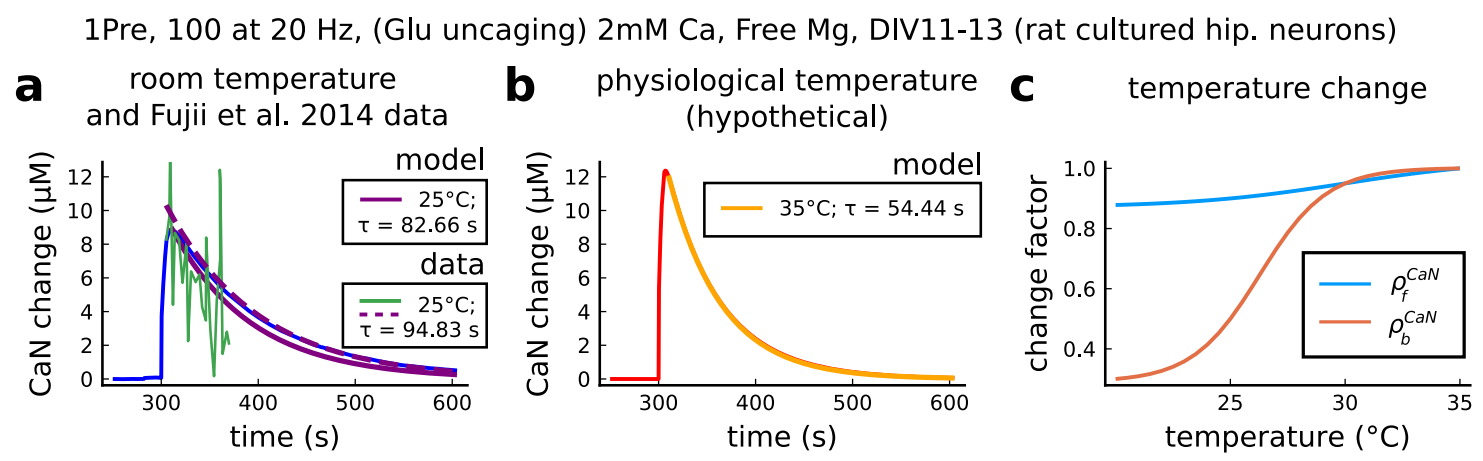

Fig. M12. I CaN temperature changes in our model caused by 1Pre, 100 at $20 \mathrm{~Hz}$ with Glutamate uncaging (no failures allowed), $2 \mathrm{Mm}$ Ca, Free Mg, 11-13 days in vitro. a, Simulated caN change (blue solid line) in response to the same stimuli of the CaN measurement from ${ }^{85} \mathrm{RY}-\mathrm{CaN}$ fluorescent probe (green solid line). The decay time $(\tau)$ estimated from data $\left(y=a \cdot e^{-t \cdot b}\right)$ is $94.83 \mathrm{~s}$ (dashed purple line) and for our model (solid purple line) is $82.66 \mathrm{~s}$. b. Simulated $\mathrm{CaN}$ change for physiological temperature with decay time $54.44 \mathrm{~s}$. Due to the lack of data, CaN kinetic change was set to fit plasticity on the protocols use in this work. c, Temperature change, $\rho_{f}^{C a N}$ and $\rho_{b}^{C a N}$, aplied to CaN association and dissociation rates. Our dissociation rate becomes $0.006 s^{-1}$ for $22^{\circ} \mathrm{C}$ ( same temperature as $i^{87}$ ), $0.0012 s^{-1}$ ), at the same temperature the association rate becomes $9.45 \cdot 10^{6} M^{-1} s^{-1}\left(46 \cdot 10^{6} M^{-1} s^{-1}\right.$ in $\left.^{87}\right)$. 
bioRxiv preprint doi: https://doi.org/10.1101/2021.03.30.437703; this version posted May 27, 2021. The copyright holder for this preprint (which was not certified by peer review) is the author/funder, who has granted bioRxiv a license to display the preprint in perpetuity. It is made available under aCC-BY-NC-ND 4.0 International license.

\begin{tabular}{|c|c|c|}
\hline REACTIONS & VALUE & REFERENCE \\
\hline \multicolumn{3}{|c|}{ Coarse-grained model, CaM-Ca reactions } \\
\hline $\begin{array}{l}\mathrm{CaM} 0+2 \mathrm{Ca} \Rightarrow \mathrm{CaM} 2 \mathrm{C} \\
\mathrm{CaM} 2 \mathrm{~N}+2 \mathrm{Ca} \Rightarrow \mathrm{CaM} 4\end{array}$ & $k_{f}^{2 C}=\operatorname{adapt}\left(k_{o n}^{1 C}, k_{o n}^{2 C}, k_{o f f}^{1 C}, k_{o n}^{2 C}, C a\right)$ & 78 \\
\hline $\begin{array}{l}\mathrm{CaM} 0+2 \mathrm{Ca} \Rightarrow \mathrm{CaM} 2 \mathrm{~N} \\
\mathrm{CaM} 2 \mathrm{C}+2 \mathrm{Ca} \Rightarrow \mathrm{CaM} 4\end{array}$ & $k_{f}^{2 N}=\operatorname{adapt}\left(k_{o n}^{1 N}, k_{o n}^{2 N}, k_{o f f}^{1 N}, k_{o n}^{2 N}, C a\right)$ & 78 \\
\hline $\begin{array}{l}\mathrm{CaM} 2 \mathrm{C} \Rightarrow \mathrm{CaM} 0+2 \mathrm{Ca} \\
\mathrm{CaM} 4 \Rightarrow \mathrm{CaM} 2 \mathrm{~N}+2 \mathrm{Ca}\end{array}$ & $k_{b}^{2 C}=\operatorname{adapt}\left(k_{o f f}^{1 C}, k_{o f f}^{2 C}, k_{o f f}^{1 C}, k_{o n}^{2 C}, C a\right)$ & 78 \\
\hline $\begin{array}{l}\mathrm{CaM} 2 \mathrm{~N} \Rightarrow \mathrm{CaM} 0+2 \mathrm{Ca} \\
\mathrm{CaM} 4 \Rightarrow \mathrm{CaM} 2 \mathrm{C}+2 \mathrm{Ca}\end{array}$ & $k_{b}^{2 N}=\operatorname{adapt}\left(k_{o f f}^{1 N}, k_{o f f}^{2 N}, k_{o f f}^{1 N}, k_{o n}^{2 N}, C a\right)$ & 78 \\
\hline & $\begin{array}{l}k_{o n}^{1 C}=5 \cdot 10^{6} M^{-1} s^{-1} \\
k_{o n}^{2 C}=10 \cdot 10^{6} M^{-1} s^{-1} \\
k_{o n}^{1 N}=100 \cdot 10^{6} M^{-1} s^{-1} \\
k_{o n}^{2 N}=200 \cdot 10^{6} M^{-1} s^{-1} \\
k_{o f f}^{1 C}=50 s^{-1} \\
k_{o f f}^{2 C}=10 s^{-1} \\
k_{o f f}^{1 N}=2000 s^{-1} \\
k_{o f f}^{2 N}=500 s^{-1}\end{array}$ & $\begin{array}{r}1.2 \text { to } 9.6 \cdot 10^{6} M^{-1} s^{-178} \\
5 \text { to } 35 \cdot 10^{6} M^{-1} s^{-178} \\
25 \text { to } 260 \cdot 10^{6} M^{-1} s^{-178} \\
50 \text { to } 300 \cdot 10^{6} M^{-1} s^{-178} \\
10 \text { to } 70 s^{-178} \\
8.5 \text { to } 10 s^{-178} \\
1 \cdot 10^{3} \text { to } 4 \cdot 10^{3} s^{-178} \\
0.5 \cdot 10^{3} \text { to }>1 \cdot 10^{3} s^{-178}\end{array}$ \\
\hline \multicolumn{3}{|c|}{ Coarse-grained model, KCaM-Ca reactions } \\
\hline $\begin{array}{l}\mathrm{KCaM} 0+2 \mathrm{Ca} \Rightarrow \mathrm{KCaM} 2 \mathrm{C} \\
\mathrm{KCaM} 2 \mathrm{~N}+2 \mathrm{Ca} \Rightarrow \mathrm{KCaM} 4\end{array}$ & $k_{f}^{K 2 C}=\operatorname{adapt}\left(k_{o n}^{K 1 C}, k_{o n}^{K 2 C}, k_{o f f}^{K 1 C}, k_{o n}^{K 2 C}, C a\right)$ & 78 \\
\hline $\begin{array}{l}\mathrm{KCaM} 0+2 \mathrm{Ca} \Rightarrow \mathrm{KCaM} 2 \mathrm{~N} \\
\mathrm{KCaM} 2 \mathrm{C}+2 \mathrm{Ca} \Rightarrow \mathrm{KCaM} 4\end{array}$ & $k_{f}^{K 2 N}=\operatorname{adapt}\left(k_{o n}^{K 1 N}, k_{o n}^{K 2 N}, k_{o f f}^{K 1 N}, k_{o n}^{K 2 N}, C a\right)$ & 78 \\
\hline $\begin{array}{l}\mathrm{KCaM} 2 \mathrm{C} \Rightarrow \mathrm{KCaM} 0+2 \mathrm{Ca} \\
\mathrm{KCaM} 4 \Rightarrow \mathrm{KCaM} 2 \mathrm{~N}+2 \mathrm{Ca}\end{array}$ & $k_{b}^{K 2 C}=\operatorname{adapt}\left(k_{o f f}^{K 1 C}, k_{o f f}^{K 2 C}, k_{o f f}^{K 1 C}, k_{o n}^{K 2 C}, C a\right)$ & 78 \\
\hline $\begin{array}{l}\mathrm{KCaM} 2 \mathrm{~N} \Rightarrow \mathrm{KCaM} 0+2 \mathrm{Ca} \\
\mathrm{KCaM} 4 \Rightarrow \mathrm{KCaM} 2 \mathrm{C}+2 \mathrm{Ca}\end{array}$ & $k_{b}^{K 2 N}=\operatorname{adapt}\left(k_{o f f}^{K 1 N}, k_{o f f}^{K 2 N}, k_{o f f}^{K 1 N}, k_{o n}^{K 2 N}, C a\right)$ & 78 \\
\hline & $\begin{array}{l}k_{o n}^{K 1 C}=44 \cdot 10^{6} M^{-1} s^{-1} \\
k_{o n}^{K 2 C}=44 \cdot 10^{6} M^{-1} s^{-1} \\
k_{o n}^{K 1 N}=76 \cdot 10^{6} M^{-1} s^{-1} \\
k_{o n}^{K 2 N}=76 \cdot 10^{6} M^{-1} s^{-1} \\
k_{o f f}^{K 1 C}=33 s^{-1} \\
k_{o f f}^{K 2 C}=0.8 s^{-1} \\
k_{o f f}^{K 1 N}=300 s^{-1} \\
k_{o f f}^{K 2 N}=20 s^{-1}\end{array}$ & $\begin{array}{r}78 \\
78 \\
78 \\
78 \\
78 \\
78 \\
0.49 \text { to } 4.9 s^{-178} \\
78 \\
6 \text { to } 60 s^{-178} \\
\end{array}$ \\
\hline \multicolumn{3}{|c|}{ Coarse-grained model, CaM-mKCaM reactions } \\
\hline$\overline{\mathrm{CaM} 0+\mathrm{mKCaM} \Rightarrow \mathrm{mKCaM} 0}$ & $k_{f}^{\mathrm{CaM0}}=3.8 \cdot 10^{3} \mathrm{M}^{-1} \mathrm{~s}^{-1}$ & 78 \\
\hline $\mathrm{CaM} 2 \mathrm{C}+\mathrm{mKCaM} \Rightarrow \mathrm{mKCaM} 2 \mathrm{C}$ & $k_{f}^{\operatorname{CaM2C}}=0.92 \cdot 10^{6} M^{-1} s^{-1}$ & 78 \\
\hline $\mathrm{CaM} 2 \mathrm{~N}+\mathrm{mKCaM} \Rightarrow \mathrm{mKCaM} 2 \mathrm{~N}$ & $k_{f}^{\text {CaM } 2 N}=0.12 \cdot 10^{6} M^{-1} s^{-1}$ & 78 \\
\hline $\mathrm{CaM} 4+\mathrm{mKCaM} \Rightarrow \mathrm{mKCaM} 4$ & $k_{f}^{C a M 4}=30 \cdot 10^{6} M^{-1} s^{-1}$ & 14 to $60 \cdot 10^{6} M^{-1} s^{-178}$ \\
\hline $\mathrm{mKCaM} 0 \Rightarrow \mathrm{CaM} 0+\mathrm{mKCaM}$ & $k_{b}^{\operatorname{CaM0}}=5.5 s^{-1}$ & 78 \\
\hline $\mathrm{mKCaM} 2 \mathrm{C} \Rightarrow \mathrm{CaM} 2 \mathrm{C}+\mathrm{mKCaM}$ & $k_{b}^{C a M 2 C}=6.8 s^{-1}$ & 78 \\
\hline $\mathrm{mKCaM} 2 \mathrm{~N} \Rightarrow \mathrm{CaM} 2 \mathrm{~N}+\mathrm{mKCaM}$ & $k_{b}^{C a M 2 N}=1.7 s^{-1}$ & 78 \\
\hline $\mathrm{mKCaM} 4 \Rightarrow \mathrm{CaM} 0+\mathrm{mKCaM}$ & $k_{b}^{\text {CaM4 }}=1.5 s^{-1}$ & 1.1 to $2.3 s^{-178}$ \\
\hline \multicolumn{3}{|c|}{ Coarse-grained model, self-phosphorylation reactions } \\
\hline $\begin{array}{l}\text { KCaM0 } \Rightarrow \text { PCaM0 } \\
\text { KCaM2N } \Rightarrow \text { PCaM2N } \\
\text { KCaM2C } \Rightarrow \text { PCaM2C } \\
\text { KCaM4 } \Rightarrow \text { PCaM4 }\end{array}$ & $k_{1}=12.6 s^{-1}$ & 77 \\
\hline Fraction of activated CaMKII & $F=C a M K I I / m K C a M_{c o n}$ & see Equation $(17)^{1 /}$ \\
\hline $\begin{array}{l}\mathrm{PCaM} 0 \Rightarrow \mathrm{P}+\mathrm{CaM} 0 \\
\mathrm{PCaM} 2 \mathrm{~N} \Rightarrow \mathrm{P}+\mathrm{CaM} 2 \mathrm{~N} \\
\mathrm{PCaM} 2 \mathrm{C} \Rightarrow \mathrm{P}+\mathrm{CaM} 2 \mathrm{C} \\
\mathrm{PCaM} 4 \Rightarrow \mathrm{P}+\mathrm{CaM} 4\end{array}$ & $k_{2}=0.33^{-1}$ & $0.33 s^{-1} ;$ adapted from ${ }^{77}$ \\
\hline $\mathrm{P} \Rightarrow \mathrm{mKCaM}$ & $k_{3}=4 \cdot 0.17 s^{-1}$ & $0.17 s^{-1}$ adapted from ${ }^{77}$ \\
\hline $\mathrm{P} \Rightarrow \mathrm{P} 2$ & $k_{4}=4 \cdot 0.041 s^{-1}$ & $0.041 s^{-1}$ adapted from ${ }^{17}$ \\
\hline $\mathrm{P} 2 \Rightarrow \mathrm{P}$ & $k_{5}=8 \cdot 0.017 s^{-1}$ & $0.017 s^{-1}$ adapted from ${ }^{77}$ \\
\hline \multicolumn{3}{|c|}{ Calcineurin model, CaM-CaM4 reactions } \\
\hline 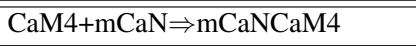 & $\bar{c}_{f}^{\mathrm{CaN}}=10.75 \cdot 10^{6} \mathrm{M}^{-1} \mathrm{~s}^{-1}$ & $46 \cdot 10^{6} M^{-1} s^{-187}$ \\
\hline $\mathrm{mCaNCaM} 4 \Rightarrow \mathrm{CaM} 4+\mathrm{mCaN}$ & $k_{b}^{C a N}=0.02 s^{-1}$ & $\begin{array}{r}0.0012 s^{-187} \\
\text { see temperature factor }\end{array}$ \\
\hline
\end{tabular}

Table M13. Parameters for the coarse-grained model published in ${ }^{78}$ and adapted by ${ }^{77}$ and this work. ${ }^{78}$ rate adaptation for the coarse-grained model $\operatorname{adapt}(a, b, c, d, C a)=\frac{a \cdot b}{c+d \cdot C a}$. 


\section{Readout}

We describe the readout mechanism which provides the plasticity event which takes place in the synapse. First, we define the following variables which are representative of "active CaMKII" and "active CaN":

Active $\mathrm{CaN}$

$$
C a N=C a N 4
$$

\section{Active CaMKII}

$$
\begin{aligned}
K C a M & =K C a M 0+K C a M 2 C+K C a M 2 N+K C a M 4 \\
P C a M & =P C a M 0+P C a M 2 C+P C a M 2 N+P C a M 4 \\
C a M K I I & =K C a M+P C a M+P+P 2 .
\end{aligned}
$$

It is known that the calcium entry initiates a cascade of events that ultimately leads to short and long term plasticity changes. Specific concentrations of CaMKII and CaN trigger activation functions $a_{c t}$ and act $_{P}$ when they belong to one of the two polygonal regions (P and $\mathrm{D})$, termed plasticity regions:

$$
\begin{aligned}
a \dot{c t} & =a_{D} \cdot \mathbb{1}_{D}-b_{D} \cdot\left(1-\mathbb{1}_{D}\right) \cdot a c t_{D} \\
\operatorname{act}_{P} & =a_{P} \cdot \mathbb{1}_{P}-b_{P} \cdot\left(1-\mathbb{1}_{P}\right) \cdot \operatorname{act}_{P} .
\end{aligned}
$$

To Specify the LTP/LTD rates, termed $D_{\text {rate }}$ and $P_{\text {rate }}$, we use the activation functions, $a c t_{D}$ and $a c t_{P}$, as follows:

$$
\begin{aligned}
P_{\text {rate }}\left(a c t_{P}\right) & =t_{P}^{-1} \frac{a c t_{P}^{h_{P}}}{a c t_{P}^{h_{P}}+K_{P}^{h_{P}}} \\
D_{\text {rate }}\left(a c t_{D}\right) & =t_{D}^{-1} \frac{a c t_{D}^{h_{D}}}{a c t_{D}^{h_{D}}+K_{D}^{h_{D}}} .
\end{aligned}
$$

The Markov plasticity chain (see Fig. M13) starts with initial conditions NC=100, LTD=0 and LTP=0. Fig. M14 shows how the readout works to predict plasticity for a single orbit. Table M14 shows the parameters to define the polygons of the plasticity regions (see Fig. M14)B.

$$
\operatorname{LTD} \underset{D_{\text {rate }}\left(a c t_{D}\right)}{\stackrel{P_{\text {rate }}\left(a c t_{P}\right)}{\rightleftharpoons}} \mathrm{NC} \underset{D_{\text {rate }}\left(a c t_{D}\right)}{\stackrel{P_{\text {rate }}\left(a c t_{P}\right)}{\rightleftharpoons}} \text { LTP }
$$

Fig. M13. I Plasticity Markov Chain. 
bioRxiv preprint doi: https://doi.org/10.1101/2021.03.30.437703; this version posted May 27, 2021. The copyright holder for this preprint (which was not certified by peer review) is the author/funder, who has granted bioRxiv a license to display the preprint in perpetuity. It is made available under aCC-BY-NC-ND 4.0 International license.
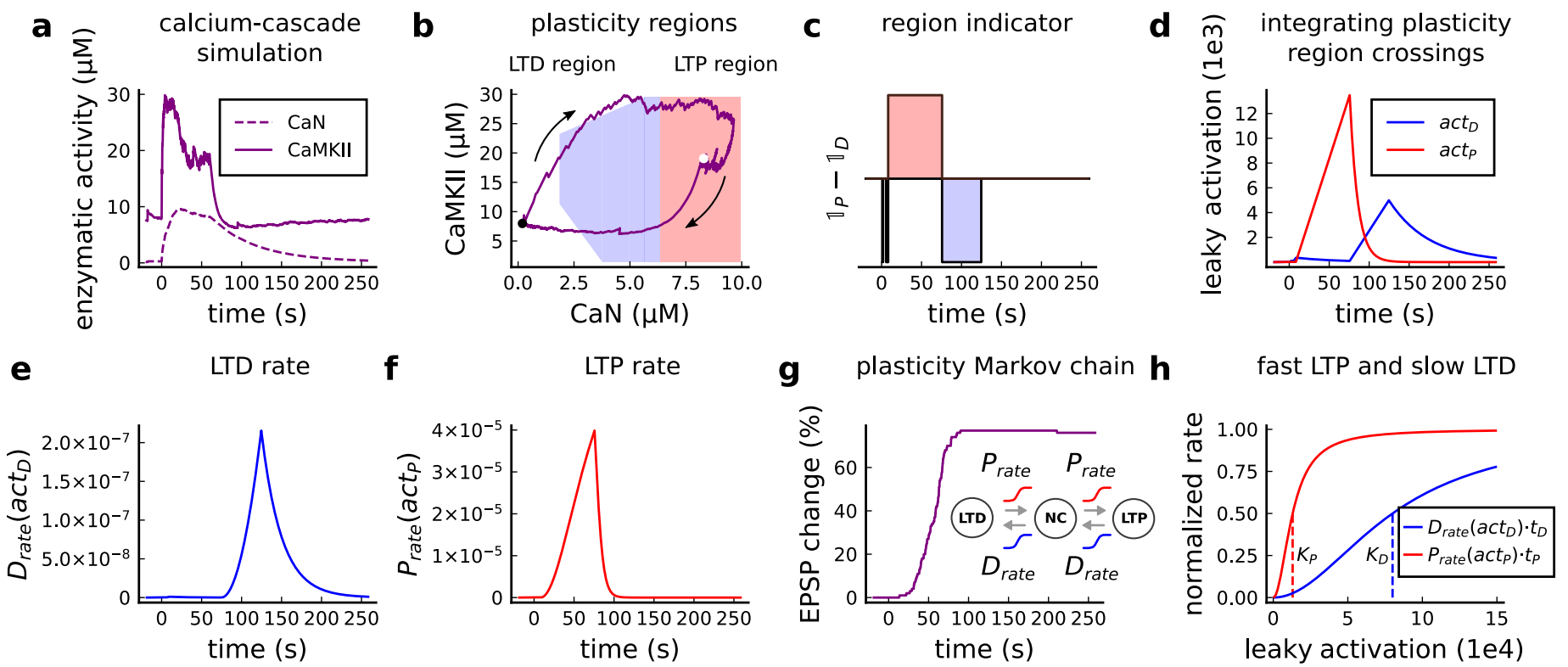

Fig. M14. I Plasticity readout for the protocol 1Pre2Post10, 300 at $\mathbf{5 H z}$, from $^{1}$. a, CaMKII and CaN activity in response to protocol 1 Pre2Post10. b, Enzymatic joint activity in the 2D plane showing LTP and LTD's plasticity regions. The black point marks the beginning of the stimulation, and the white point shows the end of the stimulation after $60 \mathrm{~s}$. c, Region indicator illustrating how the joint activity crosses the LTP and the LTD regions. d, The leaky activation functions are used respectively as input to the LTP and LTD rates. The activation function has a constant rise when the joint-activity is inside the region, and exponential decay when it is out. e, The LTD rate in response to the leaky activation function, $a c t_{D}$, in panel D. Note that this rate profile occurs after the stimulation is finished $(60 \mathrm{~s})$. The joint-activity is returning to the resting concentration in panel A. f, The LTP rate in response to the leaky activation function, $a t_{P}$, in panel D. g, Outcome of the plasticity Markov chain in response to the LTD and LTP rates. The EPSP change (\%) is estimated by the difference between the number of processes in the states LTP and LTD, LTP - LTD. h, Normalized LTP and LTD rates (multiplied to their respective time constant, $t_{D}, t_{P}$ ) sigmoids. The dashed line represents the half-activation curve for the LTP and LTD rates. Note in panel D that the leaky activation function reaches the half-activation $K_{p}=1.3 e 4$.

\begin{tabular}{|c|c|c|}
\hline NAME & VALUE & REFERENCE \\
\hline \multicolumn{3}{|l|}{ Leaking variable (a.u.) } \\
\hline rise constant inside the LTD region & $a_{D}=0.1$ a.u. $\cdot m s^{-1}$ & fitted to cover all protocols in Table M1 \\
\hline decay constant outside the LTD region & $b_{D}=2 \cdot 10^{-5}$ a.u. $\cdot m s^{-1}$ & fitted to cover all protocols in Table M1 \\
\hline decay constant outside the LTP region & $b_{P}=1 \cdot 10^{-4}$ a.u. $\cdot m s^{-1}$ & fitted to cover all protocols in Table M1 \\
\hline \multicolumn{3}{|l|}{ Plasticity Markov chain } \\
\hline LTP rate time constant & $t_{P}=1.3 \cdot 10^{4} \mathrm{~ms}$ & fitted to cover all protocols in Table M1 \\
\hline hill coefficient LTP & $h_{P}=2$ & fitted to cover all protocols in Table M1 \\
\hline hill coefficient LTD & $h_{D}=2$ & fitted to cover all protocols in Table M1 \\
\hline half occupation LTP & $K_{P}=1.3 \cdot 10^{4}$ a.u. & fitted to cover all protocols in Table M1 \\
\hline half occupation LTD & $K_{D}=8 \cdot 10^{4}$ a.u. & fitted to cover all protocols in Table M1 \\
\hline \multicolumn{3}{|l|}{ Plasticity region (edges of the polygons) } \\
\hline LTP region $(\mathrm{CaN})$ - left border & 6.35 & fitted to cover all protocols in Table M1 \\
\hline LTD region (CaMKII) - top border & 29.5 & fitted to cover all protocols in Table M1 \\
\hline LTD region (CaMKII) - bottom border & 1.4 & fitted to cover all protocols in Table M1 \\
\hline LTD region $(\mathrm{CaN})$ - right border & 1.85 & fitted to cover all protocols in Table M1 \\
\hline LTD region $(\mathrm{CaN})$ - left border & 6.35 & fitted to cover all protocols in Table M1 \\
\hline LTD region - upper diagonal (line equation in the 2D map) & $C a M K I I=+1.64 \cdot C a N+20.20$ & fitted to cover all protocols in Table M1 \\
\hline LTD region - lower diagonal (line equation in the 2D map) & CaMKII $=-5.18 \cdot C a N+20.91$ & fitted to cover all protocols in Table M1 \\
\hline
\end{tabular}

Table M14. Parameters to define the plasticity readout. 


\section{References}

1. Tigaret, C. M., Olivo, V., Sadowski, J. H., Ashby, M. C. \& Mellor, J. R. Coordinated activation of distinct $\mathrm{Ca} 2+$ sources and metabotropic glutamate receptors encodes Hebbian synaptic plasticity. Nature communications 7, 10289 (2016).

2. Inglebert, Y., Aljadeff, J., Brunel, N. \& Debanne, D. Synaptic plasticity rules with physiological calcium levels. Proceedings of the National Academy of Sciences. ISSN: 0027-8424 (2020).

3. Meredith, R. M., Floyer-Lea, A. M. \& Paulsen, O. Maturation of long-term potentiation induction rules in rodent hippocampus: role of GABAergic inhibition. Journal of Neuroscience 23, 11142-11146 (2003).

4. Wittenberg, G. M. \& Wang, S. S.-H. Malleability of spiketiming-dependent plasticity at the CA3-CA1 synapse. Journal of Neuroscience 26, 6610-6617 (2006).

5. Dudek, S. \& Bear, M. Homosynaptic long-term depression in area CA1 of hippocampus and effects of N-methyl-D-aspartate receptor blockade. Proceedings of the National Academy of Sciences 89, 4363 (1992).

6. Dudek, S. M. \& Bear, M. F. Bidirectional long-term modification of synaptic effectiveness in the adult and immature hippocampus. Journal of Neuroscience 13, 29102918 (1993).

7. Mizuno, T., Kanazawa, I. \& Sakurai, M. Differential induction of LTP and LTD is not determined solely by instantaneous calcium concentration: an essential involvement of a temporal factor. European Journal of Neuroscience 14, 701-708 (2001).

8. Sterratt, D., Graham, B., Gillies, A. \& Willshaw, D. Principles of computational modelling in neuroscience (Cambridge University Press, 2011).

9. Rizzoli, S. O. \& Betz, W. J. Synaptic vesicle pools. Nature Reviews Neuroscience 6, 57-69 (2005).

10. Rudolph, S., Tsai, M.-C., Von Gersdorff, H. \& Wadiche, J. I. The ubiquitous nature of multivesicular release. Trends in neurosciences 38, 428-438 (2015).

11. Pyle, J. L., Kavalali, E. T., Piedras-Rentería, E. S. \& Tsien, R. W. Rapid reuse of readily releasable pool vesicles at hippocampal synapses. Neuron 28, 221-231 (2000).

12. Tsodyks, M. V. \& Markram, H. The neural code between neocortical pyramidal neurons depends on neurotransmitter release probability. Proceedings of the national academy of sciences 94, 719723 (1997).

13. Hardingham, N. R. et al. Extracellular calcium regulates postsynaptic efficacy through group 1 metabotropic glutamate receptors. Journal of Neuroscience 26, 6337-6345 (2006).

14. King, R. D., Wiest, M. C. \& Montague, P. R. Extracellular calcium depletion as a mechanism of short-term synaptic depression. Journal of Neurophysiology 85, 1952-1959 (2001).
15. Fernández-Alfonso, T. \& Ryan, T. A. The kinetics of synaptic vesicle pool depletion at CNS synaptic terminals. Neuron 41, 943-953 (2004).

16. Alabi, A. A. \& Tsien, R. W. Synaptic vesicle pools and dynamics. Cold Spring Harbor perspectives in biology 4, a013680 (2012).

17. Maravall, á., Mainen, Z., Sabatini, B. \& Svoboda, K. Estimating intracellular calcium concentrations and buffering without wavelength ratioing. Biophysical journal 78, 2655-2667 (2000).

18. Forsythe, I. D., Tsujimoto, T., Barnes-Davies, M., Cuttle, M. F. \& Takahashi, T. Inactivation of presynaptic calcium current contributes to synaptic depression at a fast central synapse. Neuron 20, 797-807 (1998).

19. Südhof, T. C. The synaptic vesiclecycle revisited. Neuron 28, 317-320 (2000).

20. Spigelman, I, Tymianski, M, Wallace, C., Carlen, P. \& Velumian, A. Modulation of hippocampal synaptic transmission by low concentrations of cell-permeant $\mathrm{Ca} 2+$ chelators: effects of $\mathrm{Ca} 2+$ affinity, chelator structure and binding kinetics. Neuroscience 75, 559-572 (1996).

21. Koch, C. \& Zador, A. The function of dendritic spines: devices subserving biochemical rather than electrical compartmentalization. Journal of Neuroscience 13, 413-422 (1993).

22. Fernandez, F. R. \& White, J. A. Gain control in CA1 pyramidal cells using changes in somatic conductance. Journal of Neuroscience 30, 230-241 (2010).

23. Hines, M. L. \& Carnevale, N. T. The NEURON simulation environment. Neural computation 9, 1179-1209 (1997).

24. Golding, N. L., Kath, W. L. \& Spruston, N. Dichotomy of action-potential backpropagation in CA1 pyramidal neuron dendrites. Journal of neurophysiology $\mathbf{8 6 , 2 9 9 8 - 3 0 1 0}$ (2001).

25. Yi, G.-S., Wang, J., Deng, B. \& Wei, X.-L. Morphology controls how hippocampal CA1 pyramidal neuron responds to uniform electric fields: a biophysical modeling study. Scientific Reports 7, 1-13 (2017).

26. Mendoza, A. L., Durán, D. A. B. \& Gómez, A. B. S. Increased dendritic length in CA1 and CA3 hippocampal neurons during the metestrus phase in Wistar rats. Brain research 1682, 78-83 (2018).

27. Stuart, G., Spruston, N. \& Häusser, M. Dendrites (Oxford University Press, 2016).

28. Zhuravleva, Z., Saifullina, V. \& Zenchenko, C. Morphometric analysis of hippocampal pyramidal neurons in situ and in grafts developing in the anterior eye chambers of young and aged Wistar rats. Journal of Neural Transplantation and Plasticity 6 (1997).

29. Bartol, T. M. et al. Computational reconstitution of spine calcium transients from individual proteins. Frontiers in synaptic neuroscience 7, 17 (2015). 
30. Harris, K. M., Jensen, F. E. \& Tsao, B. Three-dimensional structure of dendritic spines and synapses in rat hippocampus (CA1) at postnatal day 15 and adult ages: implications for the maturation of synaptic physiology and long-term potentiation [published erratum appears in $\mathbf{J}$ Neurosci 1992 Aug; 12 (8): following table of contents]. Journal of Neuroscience 12, 2685-2705 (1992).

31. Adrian, M., Kusters, R., Storm, C., Hoogenraad, C. C. $\&$ Kapitein, L. C. Probing the interplay between dendritic spine morphology and membrane-bound diffusion. Biophysical journal 113, 2261-2270 (2017).

32. Popovic, M. A., Carnevale, N., Rozsa, B. \& Zecevic, D. Electrical behaviour of dendritic spines as revealed by voltage imaging. Nature communications 6, 1-12 (2015).

33. Migliore, M., Hoffman, D. A., Magee, J. C. \& Johnston, D. Role of an A-type $\mathrm{K}+$ conductance in the back-propagation of action potentials in the dendrites of hippocampal pyramidal neurons. Journal of computational neuroscience $\mathbf{7}$, 5-15 (1999).

34. Gymnopoulos, M., Cingolani, L. A., Pedarzani, P. \& Stocker, M. Developmental mapping of small-conductance calcium-activated potassium channel expression in the rat nervous system. Journal of Comparative Neurology 522, 1072-1101 (2014).

35. Grewe, B. F., Bonnan, A. \& Frick, A. Backpropagation of physiological action potential output in dendrites of slender-tufted L5A pyramidal neurons. Frontiers in cellular neuroscience 4, 13 (2010).

36. Jung, H.-Y., Mickus, T. \& Spruston, N. Prolonged sodium channel inactivation contributes to dendritic action potential attenuation in hippocampal pyramidal neurons. Journal of Neuroscience 17, 6639-6646 (1997).

37. Buchanan, K. A. \& Mellor, J. R. The development of synaptic plasticity induction rules and the requirement for postsynaptic spikes in rat hippocampal CA1 pyramidal neurones. The Journal of Physiology 585, 429-445 (2007).

38. Etherington, S. J., Atkinson, S. E., Stuart, G. J. \& Williams, S. R. Synaptic integration. eLS (2010).

39. Ebner, C., Clopath, C., Jedlicka, P. \& Cuntz, H. Unifying Long-Term Plasticity Rules for Excitatory Synapses by Modeling Dendrites of Cortical Pyramidal Neurons. Cell Reports 29, 4295-4307 (2019).

40. Mayr, C. G. \& Partzsch, J. Rate and pulse based plasticity governed by local synaptic state variables. Frontiers in Synaptic Neuroscience 2, 33 (2010).

41. Fricker, D. \& Miles, R. EPSP amplification and the precision of spike timing in hippocampal neurons. Neuron $\mathbf{2 8}$, 559-569 (2000).

42. Levine, D. \& Woody, C. Effects of active versus passive dendritic membranes on the transfer properties of a simulated neuron. Biological cybernetics 31, 63-70 (1978).

43. Kwon, T., Sakamoto, M., Peterka, D. S. \& Yuste, R. Attenuation of synaptic potentials in dendritic spines. Cell reports 20, 1100-1110 (2017).
44. Robert, A. \& Howe, J. R. How AMPA receptor desensitization depends on receptor occupancy. Journal of Neuroscience 23, 847-858 (2003).

45. Coombs, I. D., MacLean, D. M., Jayaraman, V., Farrant, M. \& Cull-Candy, S. G. Dual Effects of TARP $\gamma-2$ on Glutamate Efficacy Can Account for AMPA Receptor Autoinactivation. Cell reports 20, 1123-1135 (2017).

46. Postlethwaite, M., Hennig, M. H., Steinert, J. R., Graham, B. P. \& Forsythe, I. D. Acceleration of AMPA receptor kinetics underlies temperature-dependent changes in synaptic strength at the rat calyx of Held. The Journal of physiology 579, 69-84 (2007).

47. Spruston, N., Jonas, P. \& Sakmann, B. Dendritic glutamate receptor channels in rat hippocampal CA3 and CA1 pyramidal neurons. The Journal of physiology 482, 325-352 (1995).

48. Liu, G., Choi, S. \& Tsien, R. W. Variability of neurotransmitter concentration and nonsaturation of postsynaptic AMPA receptors at synapses in hippocampal cultures and slices. Neuron 22, 395-409 (1999).

49. Segal, M. \& Korkotian, E. Endoplasmic reticulum calcium stores in dendritic spines. Frontiers in neuroanatomy $\mathbf{8 ,} 64$ (2014).

50. Holcman, D, Korkotian, E \& Segal, M. Calcium dynamics in dendritic spines, modeling and experiments. Cell calcium 37, 467-475 (2005).

51. Sabatini, B. L., Oertner, T. G. \& Svoboda, K. The life cycle of $\mathrm{Ca} 2+$ ions in dendritic spines. Neuron 33, 439-452 (2002).

52. Hille, B. Ionic channels in excitable membranes. Current problems and biophysical approaches. Biophysical Journal 22, 283-294 (1978).

53. Chang, J.-Y. et al. CaMKII autophosphorylation is necessary for optimal integration of $\mathrm{Ca} 2+$ signals during LTP induction, but not maintenance. Neuron 94, 800-808 (2017).

54. Popescu, G., Robert, A., Howe, J. R. \& Auerbach, A. Reaction mechanism determines NMDA receptor response to repetitive stimulation. Nature 430, 790 (2004).

55. Iacobucci, G. J. \& Popescu, G. K. Kinetic Models for Activation and Modulation of NMDA Receptor Subtypes. Current Opinion in Physiology (2018).

56. Sinclair, D. et al. Effects of sex and DTNBP1 (dysbindin) null gene mutation on the developmental GluN2BGluN2A switch in the mouse cortex and hippocampus. Journal of neurodevelopmental disorders 8, 14 (2016).

57. Korinek, M, Sedlacek, M, Cais, O, Dittert, I \& Vyklicky $\mathrm{Jr}, \mathrm{L}$. Temperature dependence of N-methyl-D-aspartate receptor channels and N-methyl-D-aspartate receptor excitatory postsynaptic currents. Neuroscience $\mathbf{1 6 5}, 736-748$ (2010).

58. Maki, B. A. \& Popescu, G. K. Extracellular Ca2+ ions reduce NMDA receptor conductance and gating. Journal of General Physiology 144, 379-392 (2014). 
59. Nimchinsky, E. A., Yasuda, R., Oertner, T. G. \& Svoboda, K. The number of glutamate receptors opened by synaptic stimulation in single hippocampal spines. Journal of Neuroscience 24, 2054-2064 (2004).

60. Jahr, C. E. \& Stevens, C. F. A quantitative description of NMDA receptor-channel kinetic behavior. Journal of Neuroscience 10, 1830-1837 (1990).

61. Destexhe, A., Mainen, Z. F. \& Sejnowski, T. J. Synthesis of models for excitable membranes, synaptic transmission and neuromodulation using a common kinetic formalism. Journal of computational neuroscience 1, 195-230 (1994).

62. Griffith, T., Tsaneva-Atanasova, K. \& Mellor, J. R. Control of $\mathrm{Ca} 2+$ influx and calmodulin activation by SKchannels in dendritic spines. PLoS computational biology 12 (2016).

63. Busch, C. \& Sakmann, B. Synaptic transmission in hippocampal neurons: numerical reconstruction of quantal IPSCs. 55, 69-80 (1990).

64. Destexhe, A., Mainen, Z. F. \& Sejnowski, T. J. Kinetic models of synaptic transmission. Methods in neuronal modeling 2, 1-25 (1998).

65. Otis, T. \& Mody, I. Modulation of decay kinetics and frequency of GABAA receptor-mediated spontaneous inhibitory postsynaptic currents in hippocampal neurons. Neuroscience 49, 13-32 (1992).

66. Rinetti-Vargas, G., Phamluong, K., Ron, D. \& Bender, K. J. Periadolescent maturation of GABAergic hyperpolarization at the axon initial segment. Cell reports 20, 21-29 (2017).

67. Chamma, I., Chevy, Q., Poncer, J. C. \& Lévi, S. Role of the neuronal $\mathrm{K}-\mathrm{Cl}$ co-transporter $\mathrm{KCC} 2$ in inhibitory and excitatory neurotransmission. Frontiers in cellular neuroscience 6, 5 (2012).

68. Edwards, F. A., Konnerth, A. \& Sakmann, B. Quantal analysis of inhibitory synaptic transmission in the dentate gyrus of rat hippocampal slices: a patch-clamp study. The Journal of Physiology 430, 213-249 (1990).

69. Macdonald, R. L., Rogers, C. J. \& Twyman, R. Kinetic properties of the GABAA receptor main conductance state of mouse spinal cord neurones in culture. The Journal of Physiology 410, 479-499 (1989).

70. Magee, J. C. \& Johnston, D. Characterization of single voltage-gated $\mathrm{Na}+$ and $\mathrm{Ca} 2+$ channels in apical dendrites of rat CA1 pyramidal neurons. The Journal of physiology 487, 67-90 (1995).

71. Iftinca, $\mathrm{M}$ et al. Temperature dependence of T-type calcium channel gating. Neuroscience 142, 1031-1042 (2006).

72. Peloquin, J., Doering, C., Rehak, R \& McRory, J. Temperature dependence of Cav1. 4 calcium channel gating. Neuroscience 151, 1066-1083 (2008).

73. Higley, M. J. \& Sabatini, B. L. Calcium signaling in dendritic spines. Cold Spring Harbor perspectives in biology 4, a005686 (2012).
74. Van Herck, I. et al. Model Development of SK Channel Gating Incorporating Calcium Sensitivity and Drug Interaction. Biophysical Journal 114, 306a (2018).

75. Bock, T., Honnuraiah, S. \& Stuart, G. J. Paradoxical excitatory impact of SK channels on dendritic excitability. Journal of Neuroscience 39, 7826-7839 (2019).

76. Maylie, J., Bond, C. T., Herson, P. S., Lee, W.-S. \& Adelman, J. P. Small conductance Ca2+-activated K+ channels and calmodulin. The Journal of physiology 554, 255-261 (2004).

77. Chang, J.-Y., Nakahata, Y., Hayano, Y. \& Yasuda, R. Mechanisms of $\mathrm{Ca} 2+/$ calmodulin-dependent kinase II activation in single dendritic spines. Nature Communications 10, 2784 (2019).

78. Pepke, S., Kinzer-Ursem, T., Mihalas, S. \& Kennedy, M. B. A dynamic model of interactions of $\mathrm{Ca} 2+$, calmodulin, and catalytic subunits of $\mathrm{Ca} 2+/$ calmodulin-dependent protein kinase II. PLoS computational biology 6, e1000675 (2010).

79. Otmakhov, N., Regmi, S. \& Lisman, J. E. Fast decay of CaMKII FRET sensor signal in spines after LTP induction is not due to its dephosphorylation. PLoS One 10, e0130457 (2015).

80. Michalski, P. The delicate bistability of CaMKII. Biophysical journal 105, 794-806 (2013).

81. Kakiuchi, S. et al. Quantitative determinations of calmodulin in the supernatant and particulate fractions of mammalian tissues. The Journal of Biochemistry 92, 10411048 (1982).

82. Feng, B., Raghavachari, S. \& Lisman, J. Quantitative estimates of the cytoplasmic, PSD, and NMDAR-bound pools of CaMKII in dendritic spines. Brain research 1419, 4652 (2011).

83. Lee, S.-J. R., Escobedo-Lozoya, Y., Szatmari, E. M. \& Yasuda, R. Activation of CaMKII in single dendritic spines during long-term potentiation. Nature 458, 299304 (2009).

84. Roehrl, M. H. et al. Selective inhibition of calcineurin-NFAT signaling by blocking proteinprotein interaction with small organic molecules. Proceedings of the National Academy of Sciences 101, 7554-7559 (2004).

85. Fujii, H. et al. Nonlinear decoding and asymmetric representation of neuronal input information by CaMKII $\alpha$ and calcineurin. Cell reports 3, 978-987 (2013).

86. $\mathrm{Li}, \mathrm{H}$. et al. Balanced interactions of calcineurin with AKAP79 regulate Ca 2+-calcineurin-NFAT signaling. Nature structural \& molecular biology 19, 337 (2012).

87. Quintana, A. R., Wang, D., Forbes, J. E. \& Waxham, M. N. Kinetics of calmodulin binding to calcineurin. Biochemical and biophysical research communications 334, 674-680 (2005). 
bioRxiv preprint doi: https://doi.org/10.1101/2021.03.30.437703; this version posted May 27, 2021. The copyright holder for this preprint (which was not certified by peer review) is the author/funder, who has granted bioRxiv a license to display the preprint in perpetuity. It is made available under aCC-BY-NC-ND 4.0 International license.

\section{Supplementary files}

Supplemental files present some experiments and predictions extending the notion of parameter sensitivity. Also, they show the effect of modifications in the experimental parameters in Table M1. For instance, Fig. S1 show variations on ${ }^{1}$ 's experiment.
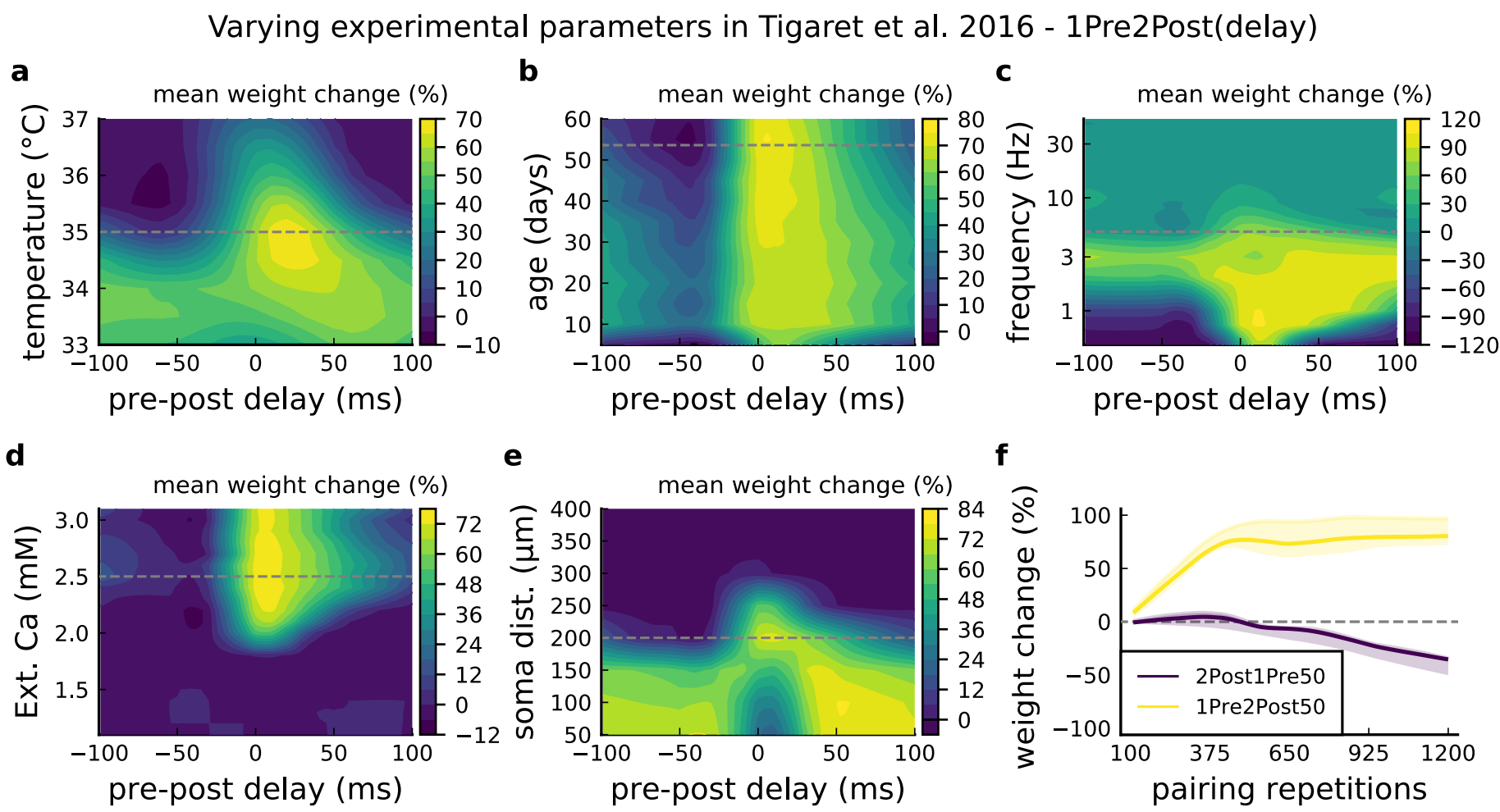

Fig. S1. I Varying ${ }^{1}$ experimental parameters. Related to Fig. . a, Mean synaptic weight change for 1Pre2Post(delay) varying the temperature, original temperature is $35^{\circ} \mathrm{C}$ (dashed grey line). b. Mean synaptic weight change for 1Pre2Post(delay) varying the age, original age is P50-55 (dashed grey line). c, Mean synaptic weight change for 1Pre2Post(delay) varying the frequency, original frequency is $5 \mathrm{~Hz}$ (dashed grey line). d, Mean synaptic weight change for 1 Pre2Post(delay) varying the $\left[\mathrm{Ca}^{2+}\right]_{\mathrm{o}}$, original $\left[\mathrm{Ca}^{2+}\right]_{\mathrm{o}}=2.5 \mathrm{mM}$ (dashed grey line). e, Mean synaptic weight change for 1Pre2Post(delay) varying the distance from the soma, original distance is $200 \mu \mathrm{m}$ (dashed grey line). A similar trend in distal spines was previously found $i^{2}$. f, Mean synaptic weight change of 1 Pre2Post50 and 2 Post1Pre50 when number of pulses increases or deacreses. Note the similarity with ${ }^{3}$ in Fig. S4C. 
Fig. S2 shows variations of ${ }^{4}$ parameters for $\left[\mathrm{Ca}^{2+}\right]_{\mathrm{o}},\left[\mathrm{Mg}^{2+}\right]_{\mathrm{o}}$, temperature and dendritic spine distance from the soma. Also, it shows the Poisson spike train protocol (as in Fig. G and H) for temperature and age parameters obtained from an estimation of the body temperature regulation during development (or thermoregulation maturation, also called maturation of temperature homeostasis, estimated in Fig. S5G).

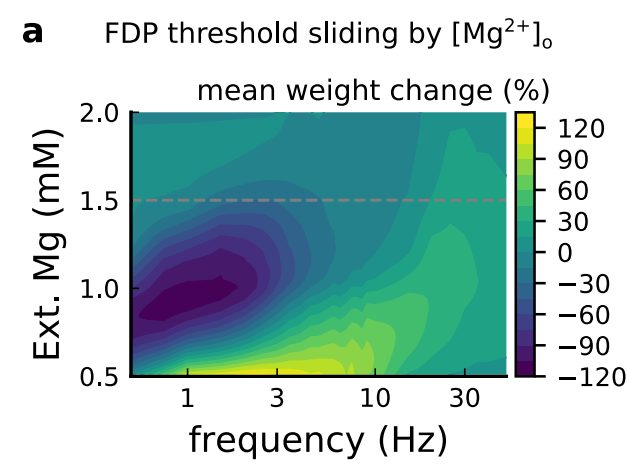

d Dudek \& Bear 19921 Pre, $900,35^{\circ} \mathrm{C}$

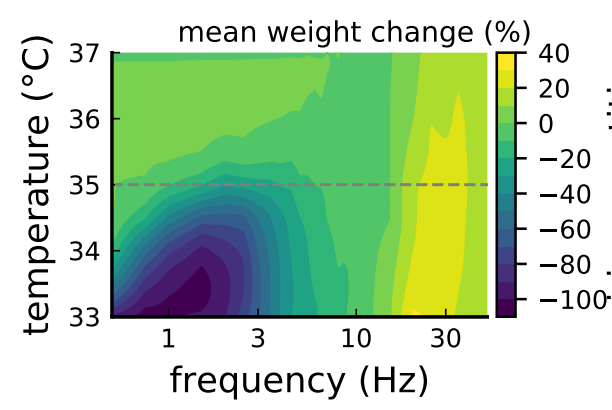

b

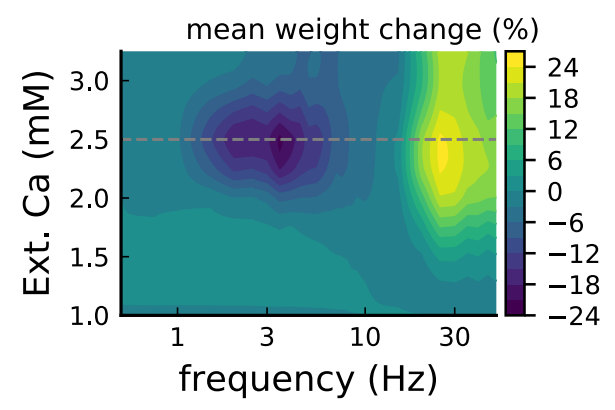

e

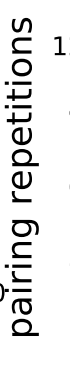

$33^{\circ} \mathrm{C}$ enhances LTD

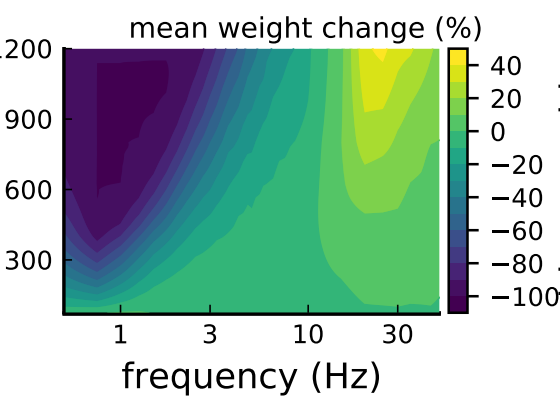

C LTD persistance in distal dendrites

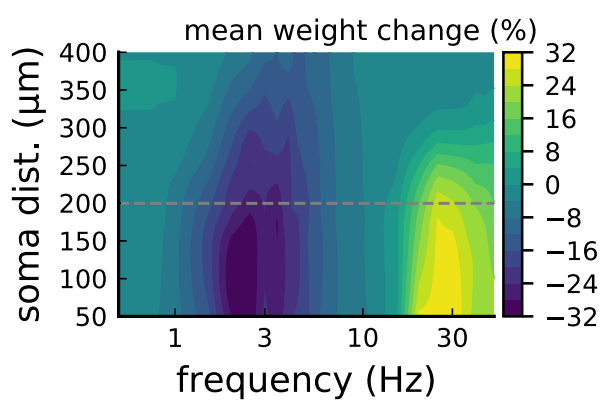

f $\quad 37^{\circ} \mathrm{C}$ abolishes LTD

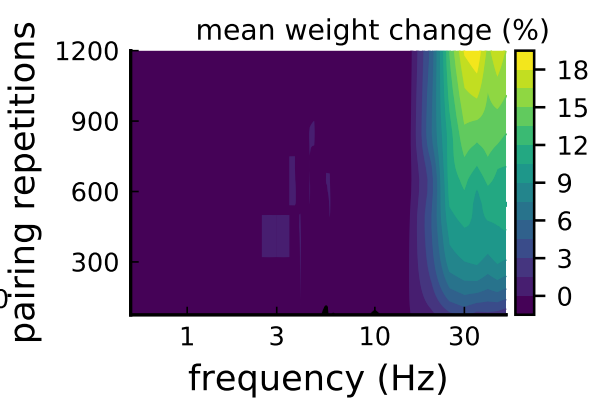

In-vivo-like firing (30s of Poissonian stimulation) following thermoregulation maturation

g

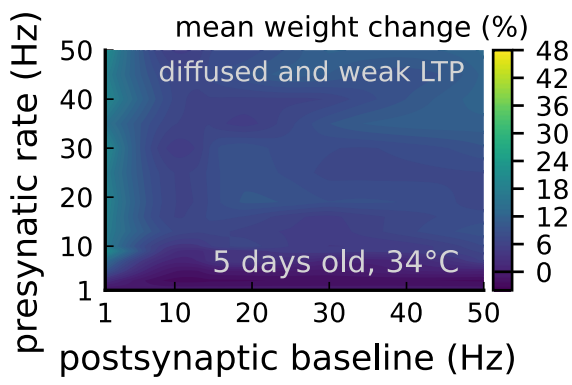

h

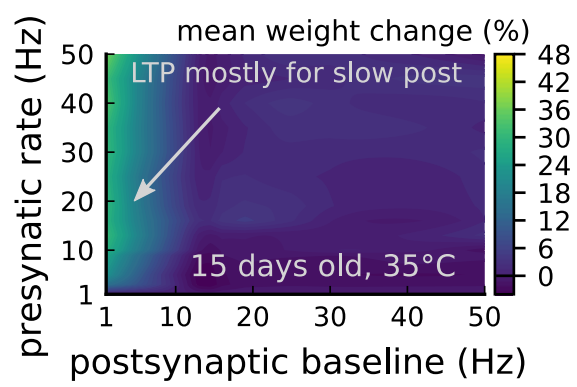

i

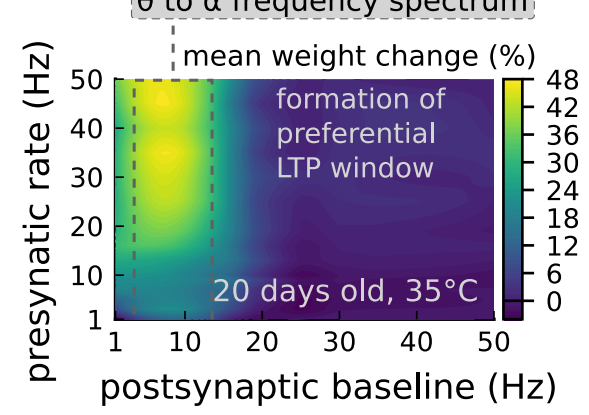

Fig. S2. I Varying experimental parameters in ${ }^{4}$ and Poisson spike train during development. Related to Fig. and . a, Mean synaptic weight change for the FDP experiment varying the $\left[\mathrm{Mg}^{2+}\right]_{0}$, original $\left[\mathrm{Mg}^{2+}\right]_{\mathrm{o}}=1.5 \mathrm{mM}$ (dashed grey line). b, Mean synaptic weight change for the FDP experiment varying the $\left[\mathrm{Ca}^{2+}\right]_{\mathrm{o}}$, original $\left[\mathrm{Ca}^{2+}\right]_{\mathrm{o}}=2.5 \mathrm{mM}$ (dashed grey line). c, Mean synaptic weight change for the FDP experiment varying the distant from the soma, original $200 \mu \mathrm{m}$ (dashed grey line). Changing the distance from the soma modifies how fast BaPs evoked by EPSP will attenuate. Note that LTD is prevalent for a spine situated far from the soma. This could justify why spines distant from the soma are smaller in size since distance correlates with synaptic weight. $\mathbf{d}$, Mean synaptic weight change for the FDP experiment varying the temperature, original temperature $35^{\circ} \mathrm{C}$ (dashed grey line). e, Mean synaptic weight change for the FDP experiment varying the pairing repetitions at $33^{\circ} \mathrm{C}$ showing how LTD is enhanced. f, Mean synaptic weight change for the FDP experiment varying the pairing repetitions at $37^{\circ} \mathrm{C}$ showing how LTD is abolished. $\mathbf{g}$, Mean synaptic weight change for pre and postsynaptic Poisson spike train during $30 \mathrm{~s}$ for $\mathrm{P} 5$ and $34^{\circ} \mathrm{C}$. The panel shows that there is weak and diffused LTP. h, Mean synaptic weight change for pre and postsynaptic Poisson spike train during $30 \mathrm{~s}$ for P15 and $35^{\circ} \mathrm{C}$. The panel shows that there is a start of LTP window forming for slow postsynaptic rates $(<1 \mathrm{~Hz})$. i, Mean synaptic weight change for pre and postsynaptic Poisson spike train during $30 \mathrm{~s}$ for $\mathrm{P} 20$ and $35^{\circ} \mathrm{C}$. The panel shows that a window forms around $10 \mathrm{~Hz}$ postsynaptic rate similar to what is shown by ${ }^{5}$ and in Fig. $\mathrm{H}$.

Fig. S3 expands the presynaptic burst strategy hypothesized to recover the LTD in adult slices (Fig. C) for 900 pairing repetitions. Also, Fig. S3 tries to isolate the contribution of each age-dependent mechanism (NMDAr, GABAr, BaP efficiency switches) for 3 and $5 \mathrm{~Hz}$ predictions in ${ }^{6}$ experiment. To this we fixed each of the three mechanisms coding for age in our model at P5 and P50, to observe how they shape the plasticity. Note the experiment in Fig. S4D-I is only to theoretically show how each age mechanism contributes to plasticity in Fig. . Also we compare predictions of between different STDP experiments across age. 
bioRxiv preprint doi: https://doi.org/10.1101/2021.03.30.437703; this version posted May 27, 2021. The copyright holder for this preprint (which was not certified by peer review) is the author/funder, who has granted bioRxiv a license to display the preprint in perpetuity. It is made available under aCC-BY-NC-ND 4.0 International license.
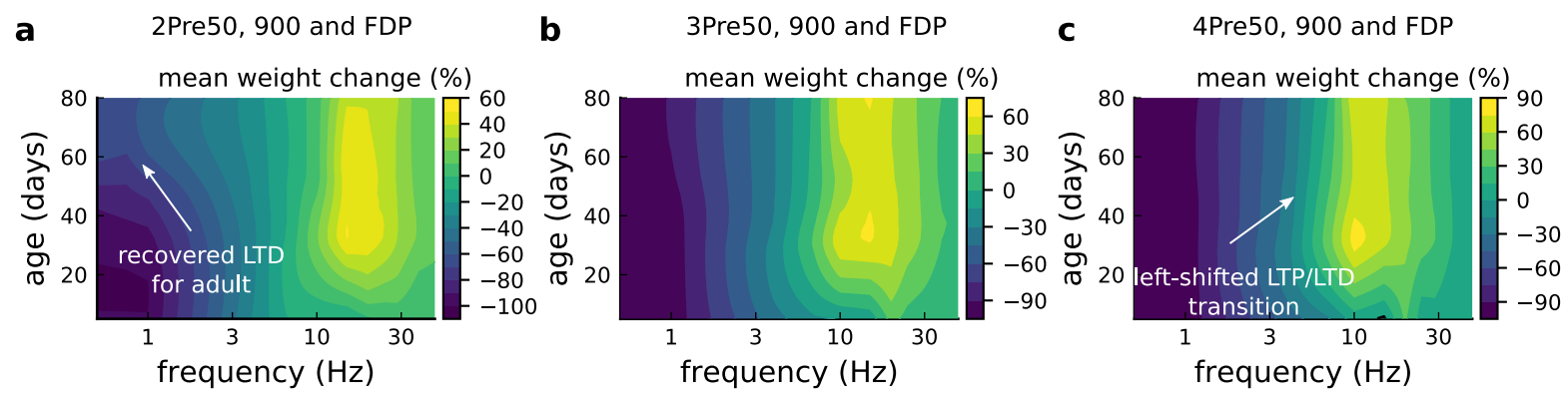

1Pre, 900 at 30 and $3 \mathrm{~Hz}$ as in Dudek and Bear (1993)
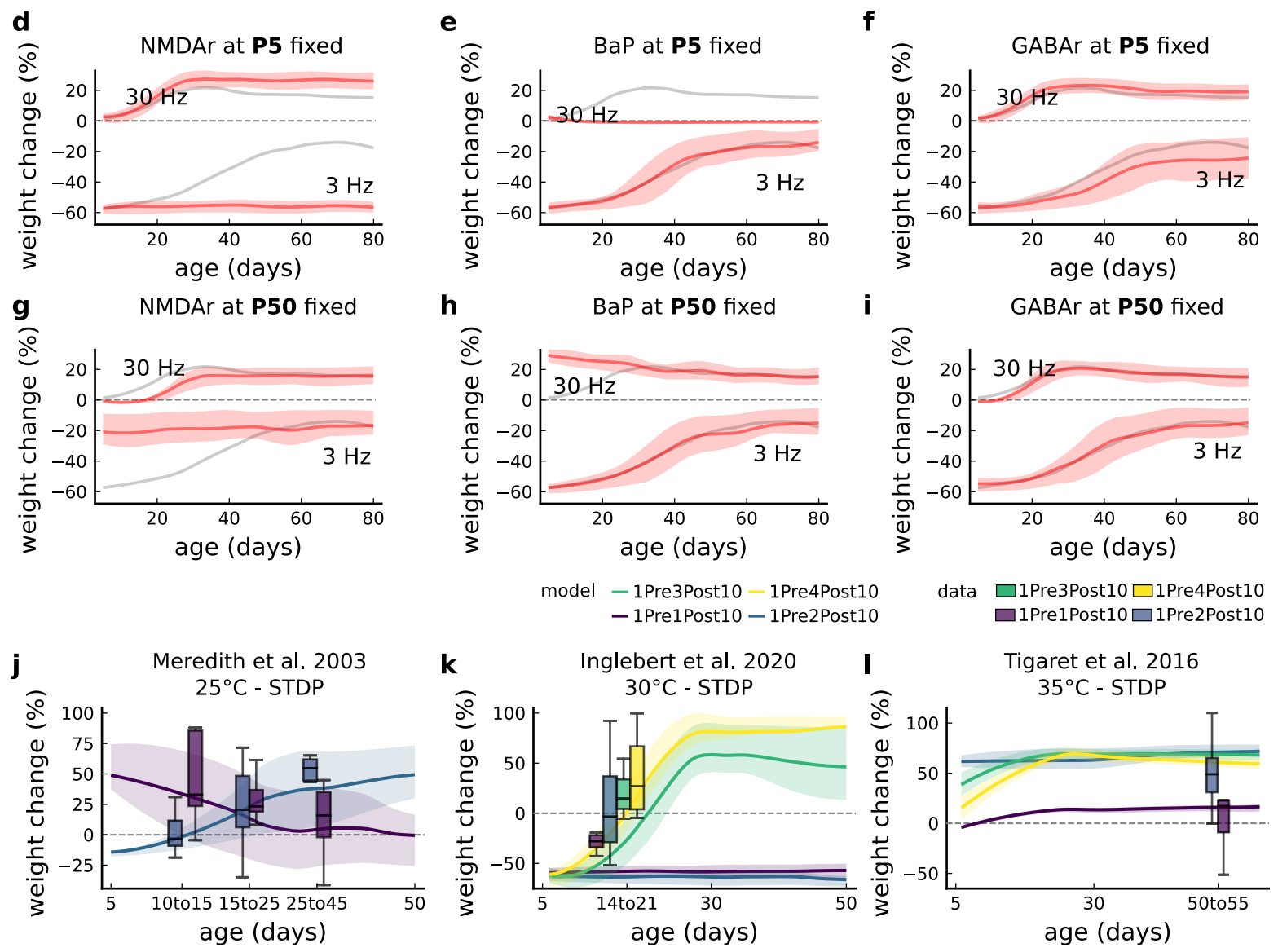

Fig. S3. I Duplets, triplets and quadruplets for FDP, perturbing developmental-mechanisms for LFS and HFS in ${ }^{6}$, and age-related changes in STDP experiments ${ }^{1,7,8}$. Related to Fig. and . a, Mean synaptic weight change (\%) for the duplet-FDP (2Pre50) experiment varying age. The panel shows showing that not only LTD is enhanced but also LTP. b, Mean synaptic weight change (\%) for the triplet-FDP (3Pre50) experiment varying age. The panel shows that LTD magnitude further enhanced for adult rats and that a leftward shift of the LTD-LTP transition. c, Mean synaptic weight change (\%) for the quadruplet-FDP (4Pre50) experiment varying age. The panel shows a further leftward shift on the LTD-LTP transition (compared to 3Pre50). d, Mean synaptic weight change (\%) for the 1 Pre 900 at 30 and $3 \mathrm{~Hz}$ with ${ }^{6}$. The panel shows the fixed NMDAr at P5 (more GluN2B) causing an increase of LTD magnitude and a slight increase of LTP magnitude for adult rats compared to baseline (grey solid line). e, Same experiment as panel D but fixing BaP maturation at P5 (higher BaP attenuation). LTP is abolished, but LTD is not affected. This is because AP induced by EPSP attenuate too fast for $30 \mathrm{~Hz}$ not able to produce enough depolarization to activate NMDAr Mg-unblock. f, Same experiment as panel D but fixing GABAr maturation at P5 (excitatory GABAr) what causes only slightly enhances LTD (3 Hz) for adult rats. g, Same experiment as panel D but fixing NMDAr at P50 (more GluN2A). LTD appears with decreased magnitude for young rats compared to baseline (grey solid line). $\mathbf{h}$, Same experiment as panel D but fixing BaP maturation at P50 (less BaP attenuation). LTP is enhanced for young rats because the BaP pairing with the slow closing GluN2B produces more calcium influx. i, Same experiment as panel D but fixing GABAr maturation at P50 (inhibitory GABAr) which does not affect the FDP experiment. j, Mean synaptic weight change (\%) for ${ }^{8}$ 's single versus burst-STDP experiment for different ages. The data from Meredith (boxplots) were pooled by the age as shown in the x-axis. The solid line represents the mean, and the shaded ribbon the 2nd and 4th quantiles simulated by the model (same for panels A-F). k, Mean synaptic weight change (\%) for ${ }^{7}$ 's STDP experiment in which the number of postsynaptic spikes increases. The $\mathrm{x}$-axis marker from 14-21 indicates that only this interval was published without further specification. We use our model to estimate age related changes to ${ }^{7}$ protocols. Note that the model does not cover the 1 Pre2Post 10 properly (model predicts only outcomes near the first data quantile). Notice that single and burst STDP leads to LTD, meanwhile ${ }^{8}$ 's to LTP or NC. 1, Mean synaptic weight change (\%) for ${ }^{1}$ 's STDP experiment which compares single versus burst STDP. The x-axis marker from 50-55 indicates that only a interval was published without further specification. We use our model to estimate age related changes to ${ }^{1}$ protocols. It is noticeable that each STDP experiment has a different development. 
bioRxiv preprint doi: https://doi.org/10.1101/2021.03.30.437703; this version posted May 27, 2021. The copyright holder for this preprint (which was not certified by peer review) is the author/funder, who has granted bioRxiv a license to display the preprint in perpetuity. It is made available under aCC-BY-NC-ND 4.0 International license.

Fig. S4 presents modifications of ${ }^{7}$ 's STDP experiment and the reproduction of ${ }^{3}$ data.

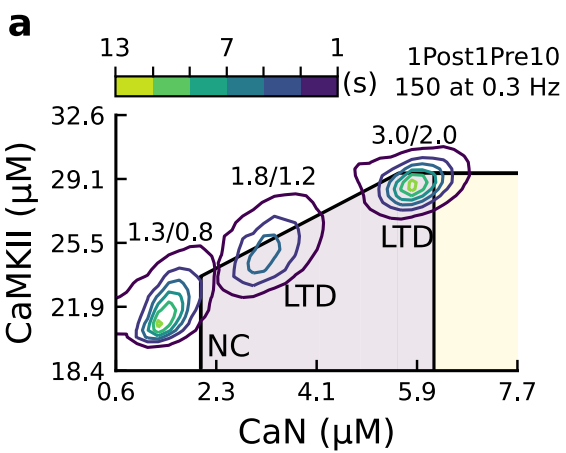

d Temperature perturbations on STDP for $2.5 \mathrm{mM} \mathrm{Ca}(\mathrm{Ca} / \mathrm{Mg}=1.5$, as Fig 6$)$

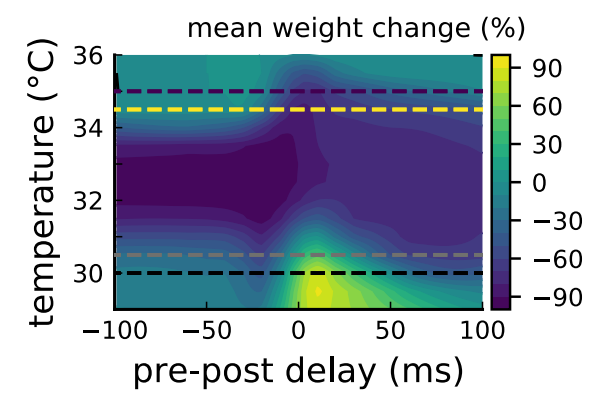

g 1Prelpost10, 100 at $0.3 \mathrm{~Hz}$ (as Fig 6)

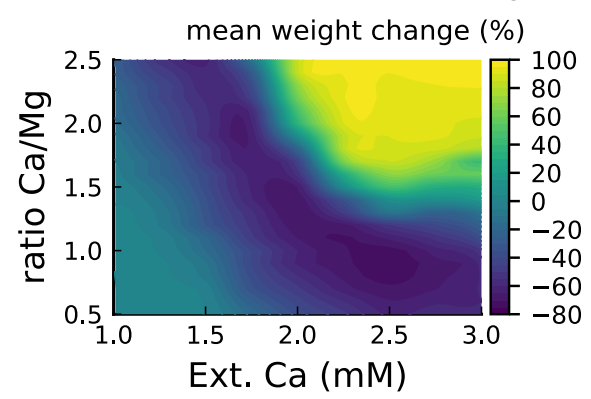

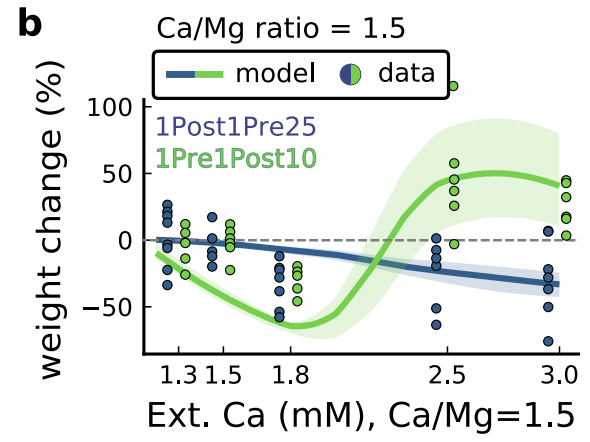

e

Physiological temperature removes
bidirectionality for $2.5 \mathrm{mM} \mathrm{Ca}(\mathrm{Ca} / \mathrm{Mg}=1.5)$

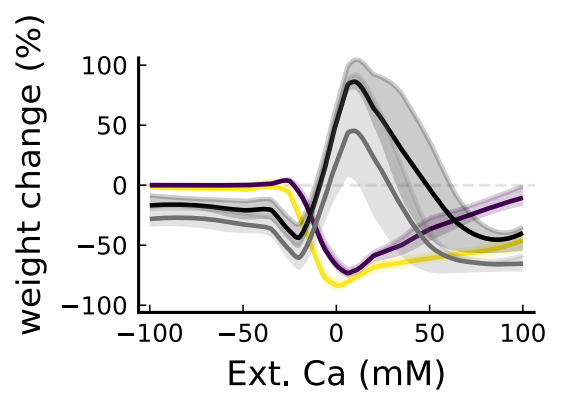

h

STDP curve for different frequencies for $2.5 \mathrm{mM} \mathrm{Ca}(\mathrm{Ca} / \mathrm{Mg}=1.5$, as Fig 6)

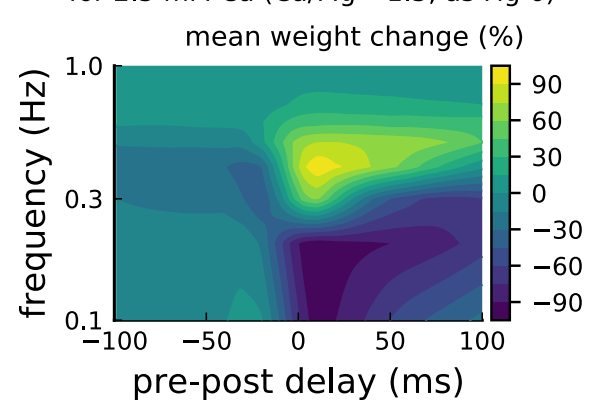

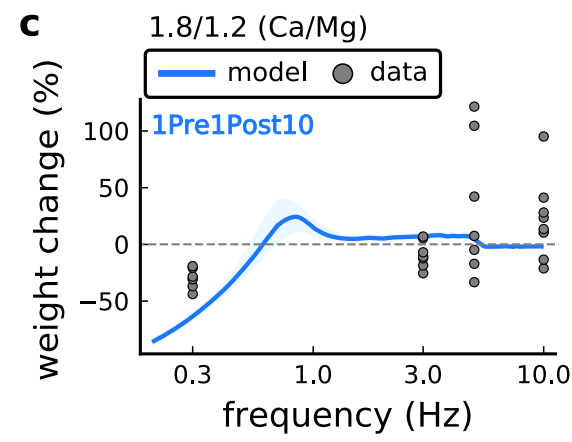

Mizuno et al. 2001 temporal factor of LTP and LTD induction

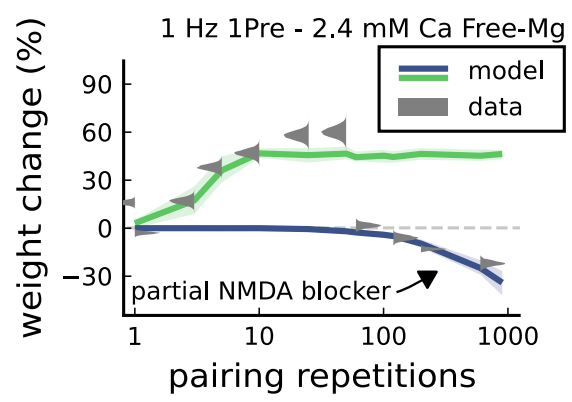

i

Weak age effect for causal STDP for $2.5 \mathrm{mM} \mathrm{Ca}(\mathrm{Ca} / \mathrm{Mg}=1.5$, as Fig 6)

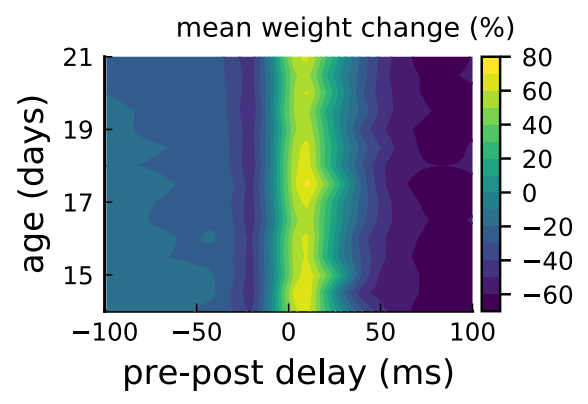

Fig. S4. I $\left[\mathrm{Ca}^{2+}\right]_{\mathrm{o}}$ and $\left[\mathrm{Mg}^{2+}\right]_{\mathrm{o}}$ related modifications for ${ }^{7}$ 's experiment. Related to Fig. . a, Mean time spent for anticausal pairing, 1Post1Pre10, at different $\mathrm{Ca} / \mathrm{Mg}$ concentrations. The contour plots are associated with the Fig. A, B and C. b, STDP and extracellular Ca/Mg. Synaptic weight change (\%) for causal (1Pre1Post10, 100 at $0.3 \mathrm{~Hz})$ and anticausal (1Post1Pre10, 150 at $0.3 \mathrm{~Hz}$ ) pairings varying $\left[\mathrm{Ca}^{2+}\right]_{0}$ from 1.0 to $3 \mathrm{mM}(\mathrm{Ca} / \mathrm{Mg}$ ratio $=1.5)$. c, Varying frequency and extracellular $\mathrm{Ca} / \mathrm{Mg}$ for the causal pairing 1 Pre1Post 10,100 at $0.3 \mathrm{~Hz}$. Synaptic weight change $(\%)$ for a single causal pairing protocol varying frequency from 0.1 to $10 \mathrm{~Hz}$. $\left[\mathrm{Ca}^{2+}\right]_{\mathrm{o}}$ was fixed at $1.8 \mathrm{mM}(\mathrm{Ca} / \mathrm{Mg}$ ratio $=1.5)$. d, Mean synaptic weight change $(\%)$ for $^{7}$ 's STDP experiment showing how temperature qualitatively modifies plasticity. The dashed lines are ploted in panel B. e, Mean synaptic weight change (\%) showing effects $0.5^{\circ} \mathrm{C}$ from panel A. Black and grey solid lines represent the same color dashed lines in panel $\mathrm{A}\left(30\right.$ and $\left.30.5^{\circ} \mathrm{C}\right)$. The bidirectional curves, black and grey lines in panel A (dashed) and panel B (solid), becoming full-LTD when temperature increases to 34.5 and $35^{\circ} \mathrm{C}$, respectively yellow and purple lines in panel A (dashed) and panel B (solid). Further increase abolishes plasticity. f, Mean synaptic weight change $(\%)$ for $^{3}$ 's experiment in Free- $\mathrm{Mg}\left(\left[\mathrm{Mg}^{2+}\right]_{\mathrm{o}}=10^{-3} \mathrm{mM}\right.$ for best fit) showing the different time requirements to induce LTP and LTD. For LTD, to simulate the NMDAr antagonist D-AP5 which causes a NMDAr partial blocking we reduced the NMDAr conductance by $97 \%$. Note the similarity with Fig. S1F. g, Mean synaptic weight change (\%) of ${ }^{7}$ 's STDP experiment changing $\left[\mathrm{Ca}^{2+}\right]_{\mathrm{o}}$ and $\mathrm{Ca} / \mathrm{Mg}$ ratio. h, Mean synaptic weight change $(\%)$ of $^{7}$ 's STDP experiment changing pre-post delay time and frequency. Note the similarity with Fig. S1C. i, Mean synaptic weight change (\%) of ${ }^{7}$ 's STDP experiment changing pre-post delay time and age. Age has a weak effect on this experiment done at $\left[\mathrm{Ca}^{2+}\right]_{\mathrm{o}}=2.5 \mathrm{mM}$. 
bioRxiv preprint doi: https://doi.org/10.1101/2021.03.30.437703; this version posted May 27, 2021. The copyright holder for this preprint (which was not certified by peer review) is the author/funder, who has granted bioRxiv a license to display the preprint in perpetuity. It is made available under aCC-BY-NC-ND 4.0 International license.

Fig.S5 shows multiple aspects related to temperature in STDP experiments and the temperature and age choices for the publications described in Table M1 compared to physiological conditions. We estimate how the rat's body temperature physiologically evolves in function of age using ${ }^{9}$ and $^{10}$ 's data.

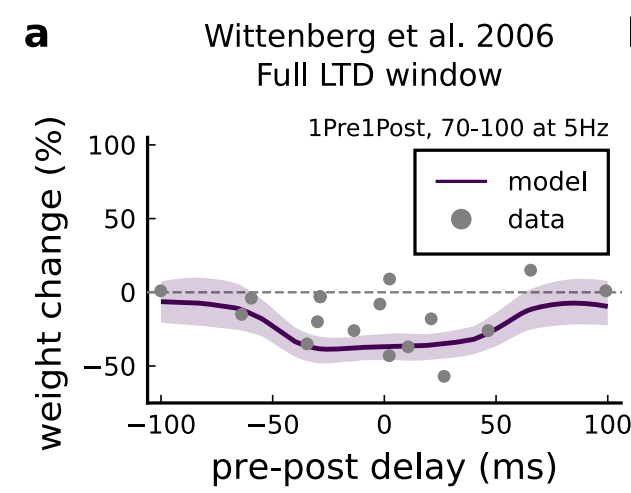

d Thermoregulation maturation

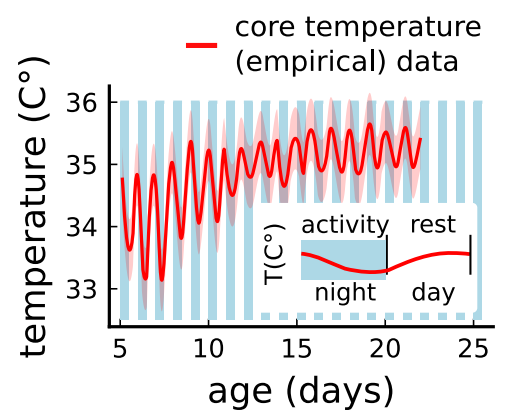

b

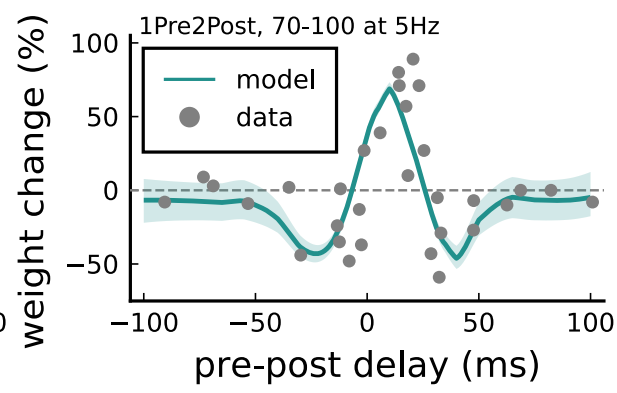

e

Wittenberg et al. 2006 Bidirectional window e

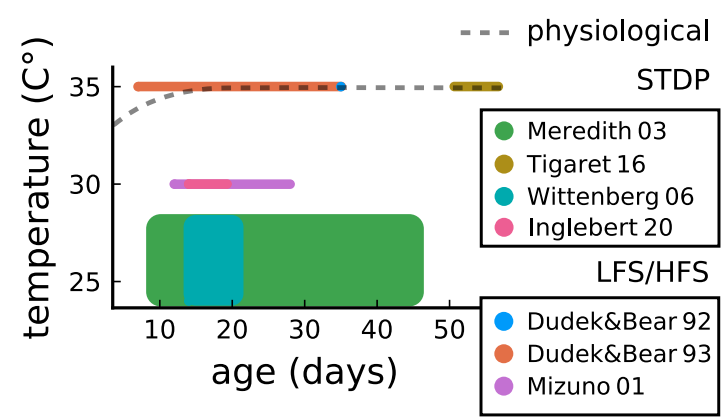

C Wittenberg et al. 2006 Full LTP window

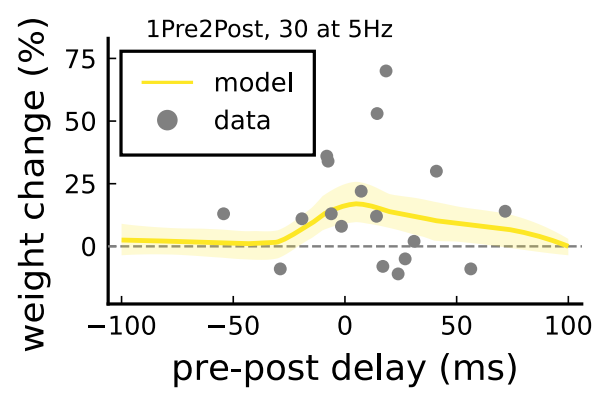

Experimental conditions for rat's CAl accute slices on plasticity literature

\section{f Initial conditions driven} by T-type noise

Fig. S5. I Age and temperature effects. Related to Fig. and . a, Mean synaptic weight change (\%) for ${ }^{11}$ 's STDP experiment for 1 Pre1Post10, $70-100$ at $5 \mathrm{~Hz}$ (see Table M1) showing a full LTD window. Our model also reproduces the fact that increasing temperature to $32-34^{\circ} \mathrm{C}$ the LTD is abolished (data not shown). b, Mean synaptic weight change (\%) for ${ }^{11}$ 's STDP experiment for 1Pre2Post10, 70-100 at $5 \mathrm{~Hz}$ (see Table M1) showing a bidirectional window. c, Mean synaptic weight change (\%) for ${ }^{11}$ 's STDP experiment for 1Pre2Post10, 20-30 at $5 \mathrm{~Hz}$ (see Table M1) showing a bidirectional window. We report that for ${ }^{11}$ experiment done in room temperature the temperature sensitivity was higher than other experiments. d, Core temperature varying with age representing the thermoregulation maturation. This function (not shown) was fitted using rat ${ }^{10}$ and mouse data ${ }^{9}$ added by $1^{\circ} \mathrm{C}$ to compensate species differences ${ }^{10}$. The blue and white bars represent the circadian rhythm as shown in ${ }^{9}$. However, the "rest rhythm" for young rats (P5-14) may vary. e, Plot showing how far from being physiological are plasticity experiments done in physiological temperatures. Suggesting, there is scarcity of physiologically relevant data to model and understand plasticity. The dashed grey line is an approximation of the mean value from panel G. f, Initial conditions for CaN-CaMKII resting concentration for different $\left[\mathrm{Ca}^{2+}\right]_{\mathrm{o}}$ and temperature values. When $\left[\mathrm{Ca}^{2+}\right]_{\mathrm{o}}$ is changed temperature is fixed at $35^{\circ} \mathrm{C}$, while temperature is changed $\left[\mathrm{Ca}^{2+}\right]_{\mathrm{o}}$ is fixed at $2 \mathrm{mM}$. 


\section{References}

1. Tigaret, C. M., Olivo, V., Sadowski, J. H., Ashby, M. C. \& Mellor, J. R. Coordinated activation of distinct $\mathrm{Ca} 2+$ sources and metabotropic glutamate receptors encodes Hebbian synaptic plasticity. Nature communications 7, 10289 (2016).

2. Ebner, C., Clopath, C., Jedlicka, P. \& Cuntz, H. Unifying Long-Term Plasticity Rules for Excitatory Synapses by Modeling Dendrites of Cortical Pyramidal Neurons. Cell Reports 29, 4295-4307 (2019).

3. Mizuno, T., Kanazawa, I. \& Sakurai, M. Differential induction of LTP and LTD is not determined solely by instantaneous calcium concentration: an essential involvement of a temporal factor. European Journal of Neuroscience 14, 701-708 (2001).

4. Dudek, S. \& Bear, M. Homosynaptic long-term depression in area CA1 of hippocampus and effects of N-methyl-D-aspartate receptor blockade. Proceedings of the National Academy of Sciences 89, 4363 (1992).

5. Graupner, M., Wallisch, P. \& Ostojic, S. Natural firing patterns imply low sensitivity of synaptic plasticity to spike timing compared with firing rate. Journal of Neuroscience 36, 11238-11258 (2016).

6. Dudek, S. M. \& Bear, M. F. Bidirectional long-term modification of synaptic effectiveness in the adult and immature hippocampus. Journal of Neuroscience 13, 29102918 (1993).

7. Inglebert, Y., Aljadeff, J., Brunel, N. \& Debanne, D. Synaptic plasticity rules with physiological calcium levels. Proceedings of the National Academy of Sciences. ISSN: 0027-8424 (2020).

8. Meredith, R. M., Floyer-Lea, A. M. \& Paulsen, O. Maturation of long-term potentiation induction rules in rodent hippocampus: role of GABAergic inhibition. Journal of Neuroscience 23, 11142-11146 (2003).

9. McCauley, J. P. et al. Circadian modulation of neurons and astrocytes controls synaptic plasticity in hippocampal area CA1. Cell reports 33, 108255 (2020).

10. Wood, T. et al. Treatment temperature and insult severity influence the neuroprotective effects of therapeutic hypothermia. Scientific reports 6, 1-12 (2016).

11. Wittenberg, G. M. \& Wang, S. S.-H. Malleability of spiketiming-dependent plasticity at the CA3-CA1 synapse. Journal of Neuroscience 26, 6610-6617 (2006). 